\title{
Characterization of mutants affecting cuticle formation in Arabidopsis thaliana
}

\author{
By: Xiaoxue Wen
}

B.Sc. (China Agriculture University, P.R.China)

\begin{abstract}
A dissertation submitted to the Faculty of Graduate Studies and Research of Carleton University, in partial fulfillment of the requirements of the degree of Master of Science in Biology
\end{abstract}

Carleton University

Ottawa ON, Canada

(c) 2009, Xiaoxue Wen 


$\begin{array}{ll}\begin{array}{l}\text { Library and Archives } \\ \text { Canada }\end{array} & \begin{array}{l}\text { Bibliothèque et } \\ \text { Archives Canada }\end{array} \\ \begin{array}{l}\text { Published Heritage } \\ \text { Branch }\end{array} & \begin{array}{l}\text { Direction du } \\ \text { Patrimoine de l'édition }\end{array} \\ \begin{array}{l}\text { 395 Wellington Street } \\ \text { Ottawa ON K1A ON4 } \\ \text { Canada }\end{array} & \begin{array}{l}395, \text { rue Wellington } \\ \text { Ottawa ON K1A ON4 } \\ \text { Canada }\end{array}\end{array}$

Your file Votre reférence

ISBN: 978-0-494-60217-1

Our file Notre neférence

ISBN: 978-0-494-60217-1

NOTICE:

The author has granted a nonexclusive license allowing Library and Archives Canada to reproduce, publish, archive, preserve, conserve, communicate to the public by telecommunication or on the Internet, loan, distribute and sell theses worldwide, for commercial or noncommercial purposes, in microform, paper, electronic and/or any other formats.

The author retains copyright ownership and moral rights in this thesis. Neither the thesis nor substantial extracts from it may be printed or otherwise reproduced without the author's permission.
AVIS:

L'auteur a accordé une licence non exclusive permettant à la Bibliothèque et Archives Canada de reproduire, publier, archiver, sauvegarder, conserver, transmettre au public par télécommunication ou par l'Internet, prêter, distribuer et vendre des thèses partout dans le monde, à des fins commerciales ou autres, sur support microforme, papier, électronique et/ou autres formats.

L'auteur conserve la propriété du droit d'auteur et des droits moraux qui protège cette thèse. $\mathrm{Ni}$ la thèse ni des extraits substantiels de celle-ci ne doivent être imprimés ou autrement reproduits sans son autorisation.
In compliance with the Canadian Privacy Act some supporting forms may have been removed from this thesis.

While these forms may be included in the document page count, their removal does not represent any loss of content from the thesis.
Conformément à la loi canadienne sur la protection de la vie privée, quelques formulaires secondaires ont été enlevés de cette thèse.

Bien que ces formulaires aient inclus dans la pagination, il n'y aura aucun contenu manquant. 


\section{Abstract}

The cuticle is a lipid-based barrier that coats the surfaces of plant aerial organs. It plays numerous essential functions for plant life, such as prevention of uncontrolled water loss and resistance to pathogen invasion. Cuticular waxes, which are largely derived from C20-C34 very-long-chain fatty acids, are a major component of the cuticle. In Arabidopsis thaliana, numerous wax-deficient mutants, termed eceriferum (cer) mutants, have been reported. I characterized a set of novel wax-deficient mutants and identified new alleles of cer1, cer3, cer4, cer6, and cer19. The novel cer6-3 allele had flat, irregularly shaped wax crystals on the stem, as opposed to cer6 null alleles that completely lacked crystals. Sequencing revealed that cer6-3 has a point mutation that changes alanine to valine at amino acid position 397 of the encoded $\beta$-ketoacyl-CoA synthase involved in elongation of C26 acyl chains. The CER19 gene is uncloned, but wax composition defects in the stems of cer19 mutants indicated a defect in elongation of C28 acyl chains. The cer19 mutants were found to have a decrease in leaf cuticular permeability at the seedling stage using a toluidine blue staining assay. I also further characterized the previously identified cer9-1 mutant and confirmed that it has a major defect in cuticular wax production that affects nearly all wax constituents in leaves and stems. Stem surface imaging by scanning electron microscopy revealed that cer9-1 had significantly reduced wax crystal density and altered crystal morphology. A major defect in the cuticular permeability of cer9-1 rosette leaves was revealed using a chlorophyll leaching assay. The gene expression patterns of 10 genes involved in wax and cutin biosynthesis were not significantly affected in cer9-1, and thus mis-regulation of these genes likely does not account for the cer9-1 phenotype. The genomic region containing 
the CER9 gene was narrowed to a $320 \mathrm{kbp}$ region on chromosome IV by positional genetic mapping. This is an important step towards the identification and molecular characterization of the CER9 gene. 


\section{Acknowledgements}

I would like to thank my supervisor Dr. Owen Rowland for offering me the opportunity to join his group. I deeply appreciate his support, advice, encouragement, understanding, and patience throughout my academic and personal life. This thesis could not have been finished without his help and guidance.

I would like to thank Dr. Shelley Hepworth and Dr. Anne-Gaelle Rolland Lagan for serving on my research advisory committee and for their helpful suggestions. I would like to thank lan Pulsifer, Sollapura Vishwanath, Christine Lowe, Mingli Xu, Tieqiang Hu and Jhadeswar Murmu for their help, support, and exchanges of knowledge and skills, all of which enriched my experience. I would also like to thank Anish Mody for his help in my research project.

Finally, I would like to thank my parents for the support they provided through my life. Special thanks go to my boyfriend Jifeng Li for his love, understanding, support, and encouragement to help me to finish this thesis. 


\section{Table of Contents}

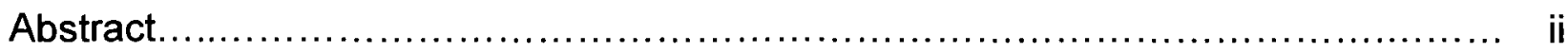

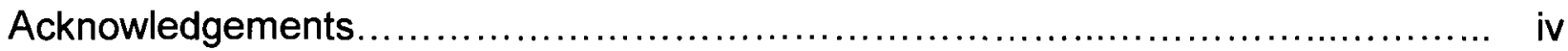

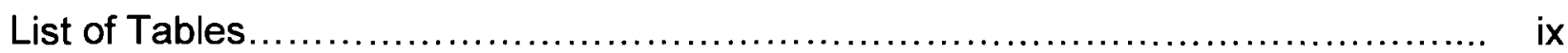

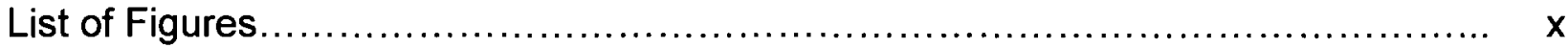

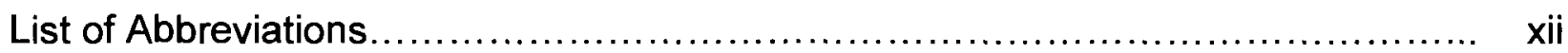

\section{Chapter 1 General Introduction}

1.1 The plant cuticle................................................................ 1

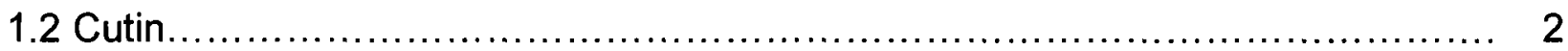

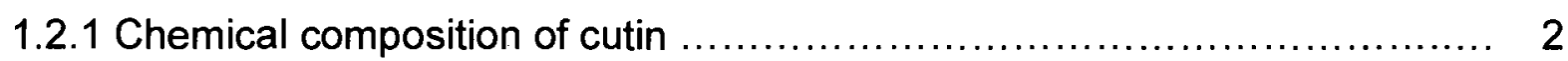

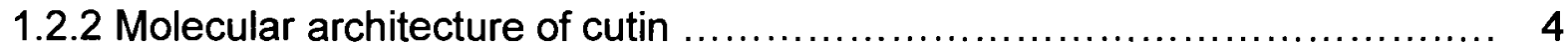

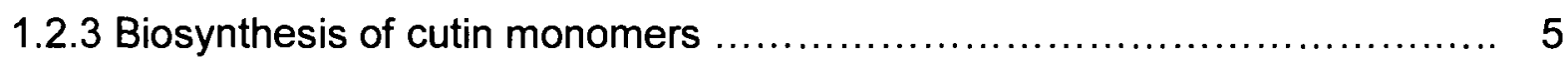

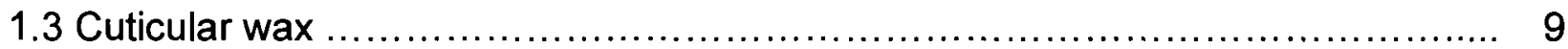

1.3.1 Chemical profiles of plant cuticular waxes.................................. 9

1.3.2 Biosynthesis of cuticular wax........................................... 10

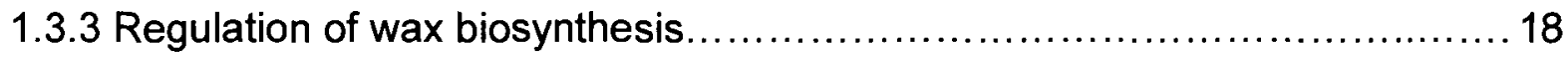

1.3.4 Transportation of cuticular wax components............................... 19

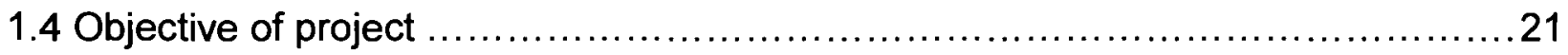

\section{Chapter 2 Identification of Novel eceriferum Mutants}

2.1 Introduction: eceriferum (cer) mutants of Arabidopsis thaliana ......................22

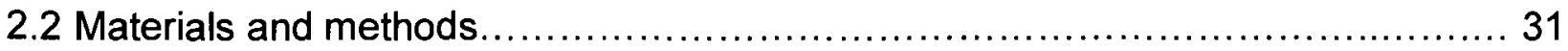

2.2.1 Plant materials and growth conditions .................................... 31

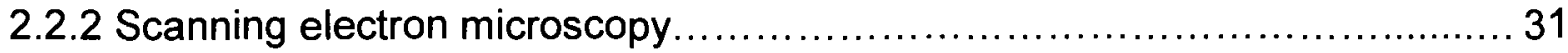




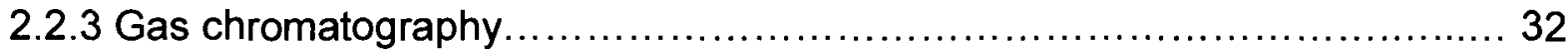

2.2.4 Complementation crosses ................................................ 33

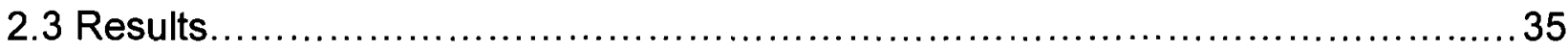

2.3.1 Survey of stem epicuticular wax crystal of cer mutants and uncharacterized

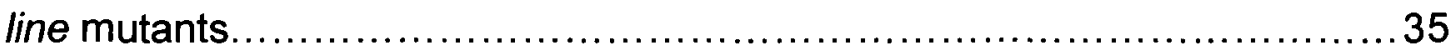

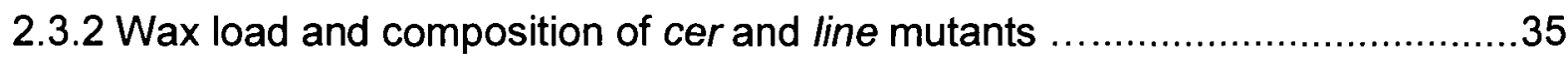

2.3.3 Comparisons of phenotypically similar cer and line mutants................. 44

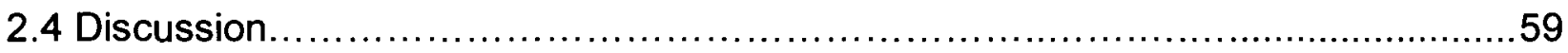

\section{Chapter 3 Characterization and Mapping of Two Novel eceriferum Mutants}

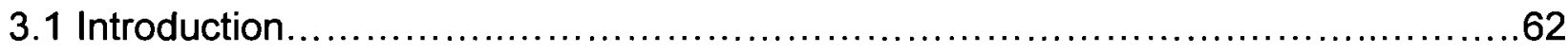

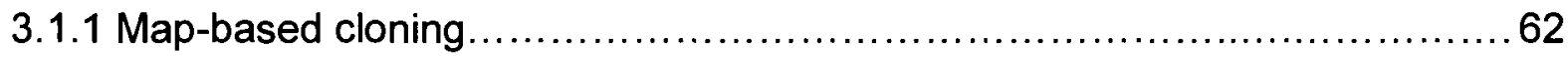

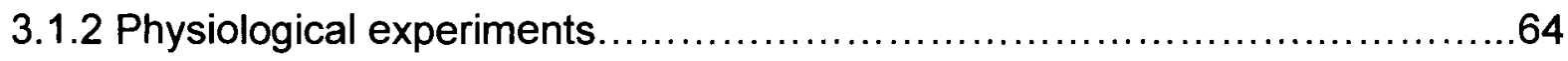

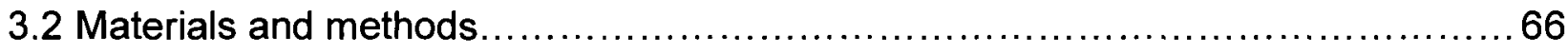

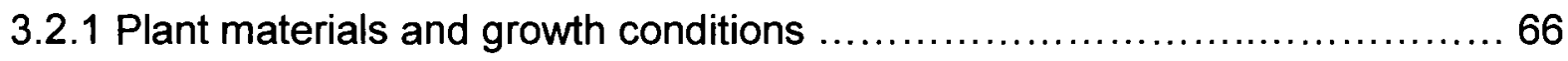

3.2.2 Plant genomic DNA extraction...........................................66

3.2.3 Strategy for designing primers for amplification of polymorphic markers ......67

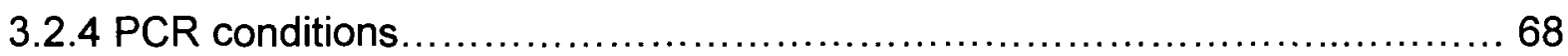

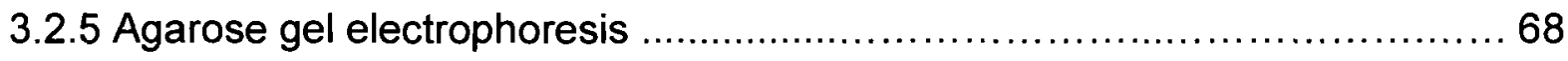

3.2.6 Mutation site verification ................................................... 69

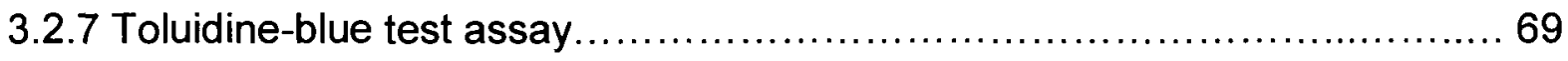

3.2.8 Fresh water loss assay ...................................................... 70

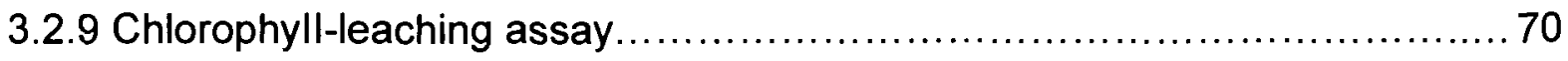

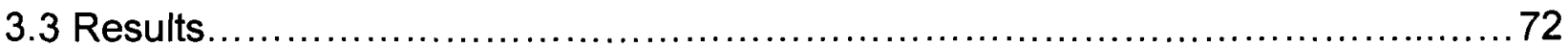


3.3.1 Rough mapping: linkage analysis of line 8 and line 85 to molecular marker... 72

3.3.2 Comparisons of epicuticular wax crystal morphology and wax composition between line 8 and cer6.

3.3.3 Sequencing of CER6 gene in the line8 mutant and alignment of predicted

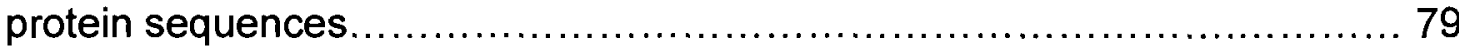

3.3.4 Complementation crosses between line8 and cer6 ........................ 79

3.3.5 Comparisons of epicuticular wax crystal morphology and wax composition between line85 and cer19............................................... 84

3.3.6 Complementation crosses between line 85 and cer19...................... 84

3.3.7 Cuticular permeability analysis ............................................. 90

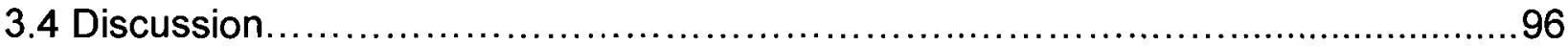

\section{Chapter 4 Genetic Mapping and Characterization of CER9}

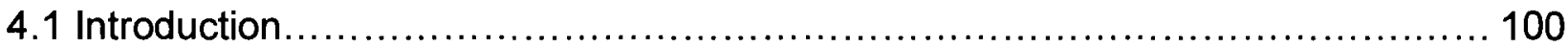

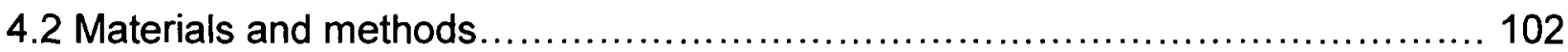

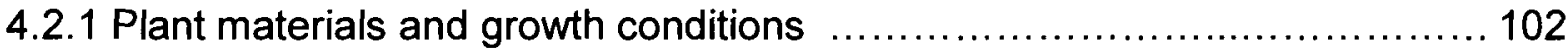

4.2.2 Generation of mapping population .................................... 102

4.2.3 Phenotypic characterization of cerg-1 ................................ 102

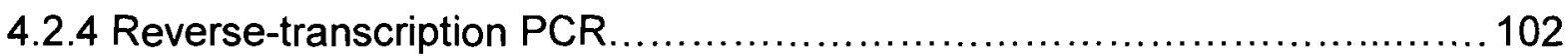

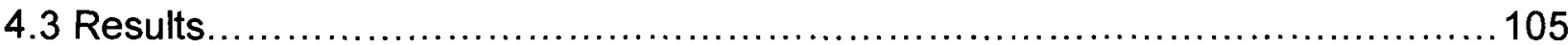

4.3.1 Analysis of epicuticular wax crystals and wax composition of cer9-1 mutants 105

4.3.2 Cuticular permeability analysis of cerg-1 ................................ 113

4.3.3 Genetic mapping of cerg-1 ................................................ 118 
4.3.4 Gene expression patterns of wax and cutin-associated genes in cer9-1..... 123

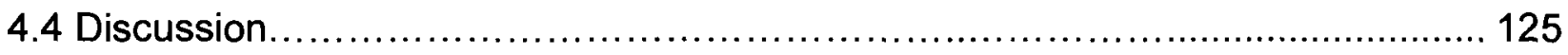

Chapter 5 General Conclusion and Discussion.............................. 128

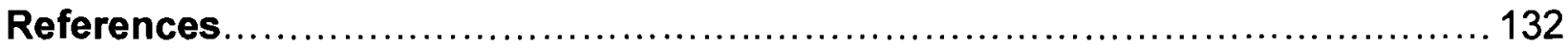




\section{List of Tables}

Table 1 Summary of all identified CER genes and other genes involved in cuticular wax

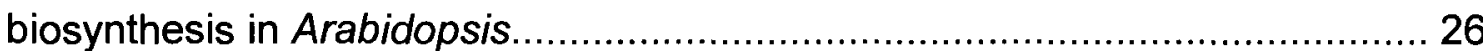

Table 2 Total stem wax load and wax composition from stems of cer mutants and wildtype ecotypes

Table 3 Total stem wax load and wax composition from stems of line mutants and wildtype ecotypes.................................................................... 43

Table 4 Complementation crosses between line and cer mutants ...........................58

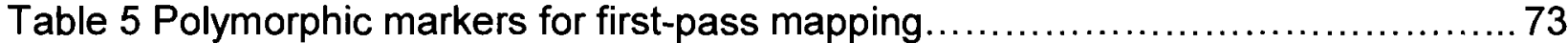

Table 6 Gene-specific primers used in RT-PCR .................................. 104

Table 7 Chemical composition of stem and leaf cuticular wax of Ler-WT and cer9-1.. 109 Table 8 Polymorphic markers for fine-pass mapping of cer9. 


\section{List of Figures}

Figure 1 Chemical structures of cutin and cuticular wax components................... 3

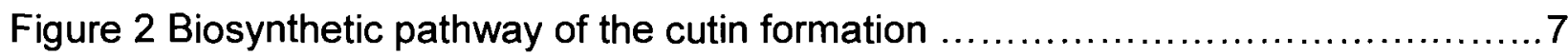

Figure 3 Proposed pathways for wax biosynthesis in Arabidopsis stems. .................11

Figure 4 Map positions of wax-deficient mutant loci on the five Arabidopsis

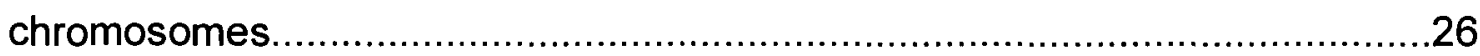

Figure 5 Model of stem cuticular wax biosynthesis in Arabidopsis..............................30

Figure 6 Scanning electron microscopy images of stem surfaces of Ler-WT and $11 \mathrm{cer}$ mutants

Figure 7 Scanning electron microscopy of stem surfaces of 10 line mutants and the corresponding wild-type ecotypes.

Figure 8 Comparison of wax crystal shape and cuticular wax composition between line6, line22, line 98 and cer4. 46

Figure 9 Comparison of wax crystal shape and cuticular wax composition between line11, line13, line14, and cer3.

Figure 10 Comparison of wax crystal shape and cuticular wax composition between line28 and cer1.

Figure 11 Comparison of wax crystal shape and cuticular wax composition between line23 and cer7.

Figure 12 Physical map positions of polymorphic markers for first-pass mapping.... .74

Figure 13 Comparison of wax crystal shape and cuticular wax composition between line 8 and cer6. 76

Figure 14 Amino acid sequence comparison of CER6(A397V), CER6(WT), KCS17, 
FAE1, LfKCS45, and StKCS6

Figure 15 Stem phenotypes of wild-type, cer6 and F1 plants from complementation crosses between line 8 and cer6 83

Figure 16 Comparison of wax crystal shape and cuticular wax composition between line 85 and cer19. 86

Figure 17 Stem phenotypes of wild-type, line85, cer19 and F1 plants from complementation crosses between line 85 and cer19. 89

Figure 18 Toluidine blue staining of Ler-WT, line85, and cer19 seedlings 92

Figure 19 Rate of water loss in fresh tissue of cer19-1, cer19-2, and Ler-WT 93

Figure 20 Chlorophyll-leaching assay of cer19-1, cer19-2, and Ler-WT leaves .95

Figure 21 Altered epicuticular wax crystals on stems of cer9-1. 106

Figure 22 Stem and leaf cuticular wax compositions of cer9-1 and the corresponding wild type ecotype (Ler-WT). 110

Figure 23 Toluidine blue staining on leaves of Ler-WT and cer9-1 seedlings. 114

Figure 24 Cuticular transpiration of Ler-WT and cer9-1. 115

Figure 25 Rate of chlorophll leaching in cer9-1 and Ler-WT leaves 117

Figure 26 Genetic mapping of CER9 the locus 120

Figure 27 RT-PCR analysis of transcripts encoding proteins involved in wax and cutin biosynthesis in Ler-WT and cer9-1. 124 


\section{List of Abbreviations}

ABC: ATP binding cassette

ABRC: Arabidopsis Biological Resource Center

ACP: acetyl-acyl carrier protein

BSTFA: bis-(trimethylsily)trifluoroacetamide

CAPs: cleaved amplified polymorphic sequences

CER: ECERIFERUM

CO: carbon monoxide

dCAPs: dereived cleaved amplified polymorphic sequences

DGAT: diacylglycerol acyltransferase

ECR: enoyl-CoA reductase

ER: endoplasmic reticulum

FAE: fatty acid elongase

FAR: fatty acyl-CoA reductase

FAS: fatty acid synthase complex

FAT: fatty acyl-ACP thioesterase

FID: flame ionization detector

FT-IR: fourier-transform infrared

GC: gas chromatography

GC-MS: gas chromatography-mass spectroscopy

GFP: green fluorescent protein

GPAT: glycerol-3-phosphate acyltransferase

HCD: hydroxyacyl-CoA dehydratase $n$ 
KAS: ketoacyl-ACP synthase

KCR: $\beta$-keto acyl reductase

KCS: $\beta$-keto acyl-CoA synthase

LACS: long-chain acyl-CoA synthetase

LCR: LACERATA

LD-RI: Lister and Dean recombinant inbred

LTP: lipid transfer protein

LTPG: glycosylphosphatidylinositol-anchored lipid transfer protein

MAH: mid-chain alkane hydroxylase

NMR: nuclear magnetic resonance

PCR: polymerase chain reaction

PM: plasma membrane

SEM: scanning electron microscopy

SSLP: simple sequence length polymorphism

TB: toluidine blue

VLCFA: very-long-chain fatty acid

WS: wax synthase

WS/DGAT: wax synthase/acyl-CoA diacylglycerol acyl-transferase 


\section{Chapter 1 General Introduction}

\subsection{The plant cuticle}

The cuticle is a lipophilic layer that coats the aerial parts of vascular plants. It is synthesized and secreted by epidermal cells. The cuticle structure varies widely among plant species, organ types and their developmental states (Jeffree, 1996, 2006). Despite this variability, all cuticles mainly consist of two types of lipophilic components: 1) cutin, a cross-linked polymer of $C 16$ and $C 18$ chain length fatty acid derivatives, and 2) cuticular wax, a mixture of very-long-chain fatty acids and derivatives that are not polymerized and that can be readily extracted by organic solvents such as chloroform or hexane.

Three distinct layers of the cuticle can be easily identified by transmission electron microscopy (from exterior inwards): epicuticular wax crystalloids, the 'cuticle proper', and the 'cuticular layer' (Kunst and Samuels, 2003). Intracuticular waxes are embedded in the cutin matrix of the cuticle proper, while epicuticular waxes lie above on the outer surface, often in the form of crystals that give the plant a whitish appearance.

The main function of the plant cuticle is to prevent non-stomatal water loss (Kerstiens, 1996; Schreiber et al., 1996). Cutin, owing to the covalent linkages between its monomers, forms the structural backbone of the cuticle (Samuels et al., 2008). The epicuticular wax layer plays important roles in the interaction of the plant with its environment. It repels rainwater, which minimizes the deposition of dust particles, pollen and spores (Holloway, 1969a, b, 1970). The cuticle serves to protect underlying tissues from mechanical damage and from excess levels of ultraviolet radiation (Krauss et al., 1997). It serves as a barrier against bacterial and fungal pathogens (Jenks et al., 1994; 
Riederer, 2006) and mediates interactions with insects (Eigenbrode and Espelie, 1995). Furthermore, the cuticle plays pivotal roles in cell-cell interactions, such as preventing fusion of cell walls from adjacent organs during plant development (Sieber et al., 2004).

\subsection{Cutin}

\subsubsection{Chemical composition of cutin}

Cutin is the insoluble polymeric component of the cuticle (Kolattukudy, 1981). It is a major constituent (typically $40-80 \%$ by weight) of the cuticle. It functions as a densely networked structural support and interacts with the epidermal cell wall.

In the 1970s, the major cutin monomers were identified by using chemical degradation (Kolattukudy, 2001). The monomeric constituents of cutin are mainly C16:0 and C18:0 fatty acids, which are typically hydroxylated (Kolattukudy, 1981) (Figure 1A). The predominant components of the C16 family of monomers are $9-$ or $10,16-$ dihydroxyhexadecanoic acid and 16-hydroxyhexadecanoic acid. Major components of the C18 family of monomers are 18-hydroxy-9, 10-epoxyoc-tadecaonoic acid and 9,10 , 18-trihydroxyoctadecanoic acid (Heredia, 2003). The chemical composition of cutin varies between organ types and location, and even varies the adaxial or abaxial surfaces of the same leaf (Holloway, 1982). For example, cutin of apple fruit contains both $\mathrm{C} 16$ and $\mathrm{C} 18$ families of monomers, while in flowers the $\mathrm{C} 18$ monomers are almost undetectable (Stark and Tian, 2006).

In Arabidopsis, the major depolymerization products of cutin are unusual because they consist of high amounts of $\mathrm{C} 16$ and $\mathrm{C} 18$ dicarboxylic acids, such as $\alpha, \omega$ dicarboxylic fatty acids and 2-hydroxy fatty acids (Bonaventure et al., 2004; Xiao et al., 2004; Franke 


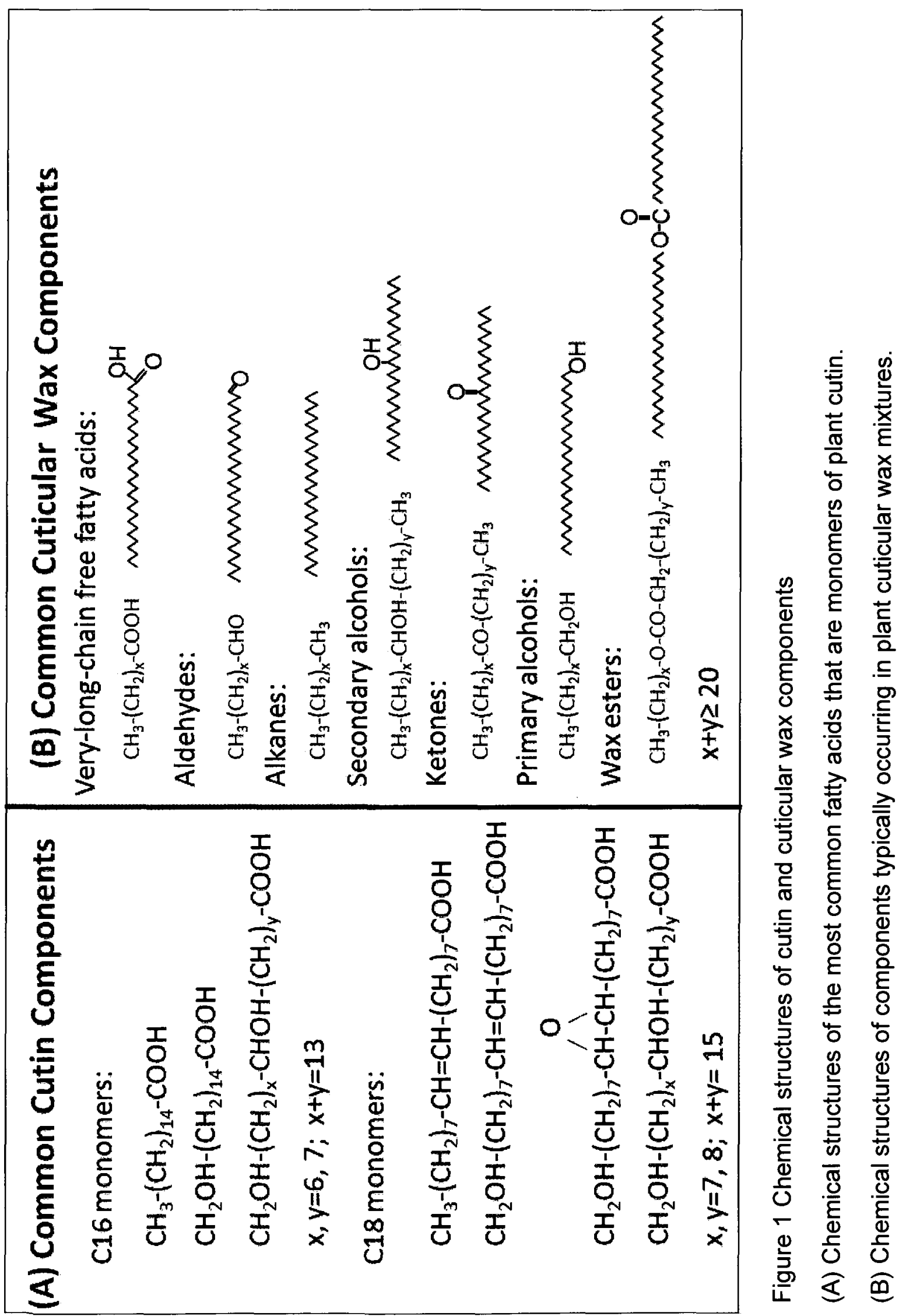


et al., 2005). Most of the cutin monomers from Arabidopsis only carry carboxyl groups, and therefore other hydroxylated fatty acids and glycerol are required to generate the cross-linked matrix (Graca et al., 2002).

In addition to the $\mathrm{C} 16$ and $\mathrm{C} 18$ major cutin monomers, numerous minor components have been found in cutin of various plant species. For example, hydroxy fatty acids that are longer and shorter than the major components, mid-chain hydroxylated compounds that do not contain a w-hydroxy group, and mid-chain hydroxylated dicarboxylic acids (Holloway et al., 1972; Hunneman and Eglington, 1972;

Deas et al., 1974; Caldicott and Eglington, 1976).

Cutan is the residual material left over when all the cuticular wax and cutin materials have been removed (Heredia, 2003). It is thought to represent cutin monomers that are held together by non-ester bonds and thus are not readily depolymerized. The ratio of cutin and cutan amounts varies between the different stages of cuticle development (Tegelaar et al., 1991). However, the structure, chemical composition, and functions of cutan are not clear.

\subsubsection{Molecular architecture of cutin}

Cutin is cross-linked in three dimensions mainly by ester bonds (Heredia, 2003). Also, half of the mid-chain hydroxyl groups in the polymer are involved in cross-linking. Most of the primary hydroxyl groups are involved in ester bonds. A very low number of unesterified carboxyl functional groups exists (Heredia, 2003). Other studies indicate that the $\omega$-hydroxy fatty acids can be polymerized to form a linear structure and that the mid-chain hydroxyl group can be esterified with carboxy groups of other fatty acids to form a dendri-form polymerized structure (Ray et al., 1998a, b). 
Since cutin soluble products are obtained by chemical depolymerization for subsequent gas chromatography-mass spectroscopy (GC-MS) analysis, they do not represent the properties or condition of the intact cutin matrix. Fourier-transform infrared (FT-IR) spectroscopical analysis and X-ray diffraction analysis have been performed to analyze the intact cuticle, which indicate that cutin has an amorphous structure with two hydrophobic interplanar spaces (Luque et al., 1995; Villena et al., 2000). Additionally, solid-state nuclear magnetic resonance (NMR) confirmed the classical chemical studies that the cutin polyester is held together mostly by primary alcohol ester linkages (Zlotnik-Mazori et al., 1988). Moreover, molecular dynamics calculations have been applied and suggest that cutin is a moderately flexible netting with motional constrains mainly located at the cross-link sites of functional ester groups (Matas et al., 1999).

\subsubsection{Biosynthesis of cutin monomers}

The initial step of cutin monomer biosynthesis is the synthesis of C16 and C18 long chain fatty acids in the plastid. The $\mathrm{C} 16$ and $\mathrm{C} 18$ fatty acid precursors are likely shared with wax biosynthesis and other cellular lipid metabolism, such as membrane lipid production. The second step of cutin biosynthesis is oxidative modification, which generates the variety of cutin monomers.

The biosynthetic pathways of both the $\mathrm{C} 16$ and $\mathrm{C} 18$ family of fatty acids were determined using radio-labeled precursors and identification of intermediates leading to cutin monomers (Kolattukudy, 1981). In Vicia sativa, studies indicate that the oxygenated octadecanoates are derived from oleic acid and linoleic acid, which undergo $\omega$-hydroxylation to form diol 9, 10-dihydroxyoctadecanoic acid and then followed by 
enantioselective epoxidation of the double bond, and finally hydroxylation of the latter functional group (Kolattukudy et al., 1981; Pinot et al., 1992) (Figure 2).

The identities of the enzymes involved in cutin biosynthesis remain elusive, but recently several key proteins required for the synthesis of cutin have been identified: fatty acyl oxidases (CYP86 family) (Benveniste et al., 2001; Wellesen et al., 2001; Xiao et al., 2004; Rupasinghe et al., 2007; Li et al., 2007, Molina et al., 2008), fatty acylactivation enzymes (LACS family) (Schnurr et al., 2004; Bessire et al., 2007), and acyltransferases (GPAT family) (Li et al., 2007).

A cytochrome P450-dependent $\omega$-hydroxylase fatty acyl oxidase is proposed to catalyze the epoxidation process (Heredia, 2003). Two genes that encode this class of enzyme have been found to be important for Arabidopsis cutin production: LACERATA (LCR, At2g45970) (Wellesen et al., 2001) and ATT1 (At4g00360) (Xiao et al., 2004). Icr mutants display a series of developmental abnormalities, such as postgenital organ fusions indicative of a cutin defect (Wellesen et al., 2001). The cloned LCR gene encodes for cytochrome P450 monooxygenase, CYP86A8, which is able to catalyze $\omega$ hydroxylation of fatty acids ranging from $\mathrm{C} 12: 0$ to $\mathrm{C} 18: 1$ in vitro (Wellesen et al., 2001). att1 (aberrant induction of type three genes) mutants show reduced cutin monomer amounts, a loose cuticle membrane ultrastructure and increased permeability to water vapor, indicating that ATT1 plays an important role in the biosynthesis of cutin monomers (Xiao et al., 2004). Cloning of the ATT1 gene indicated that it encoded CYP86A2, a cytochrome P450 monooxygenase catalyzing fatty acid oxidation (Xiao et al., 2004). 


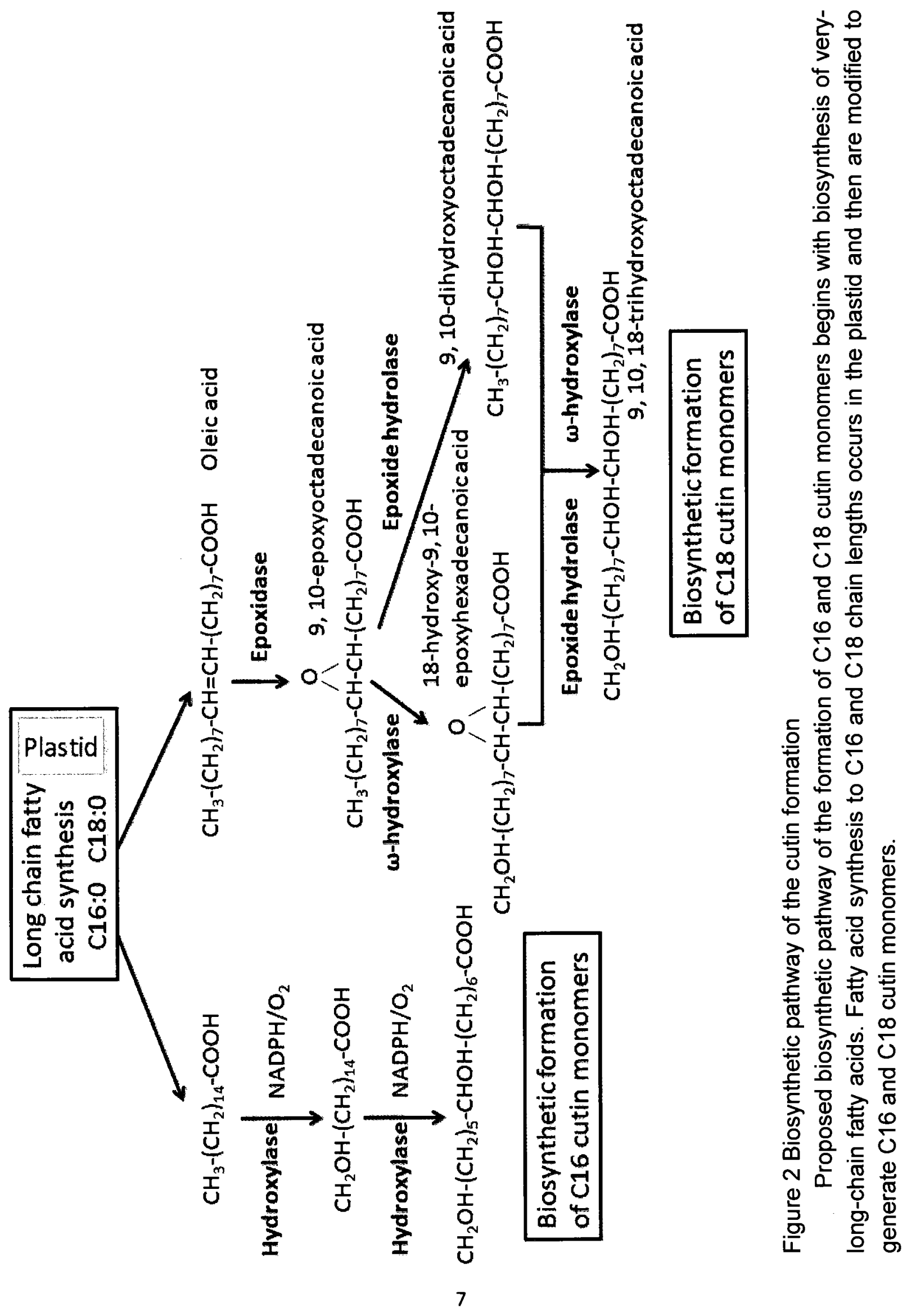


Glycerol-3-phosphate acyltransferase (GPAT) 4 and GPAT8 have been identified as being important for cutin biosynthesis. Double knockout gpat4 gpat8 mutants display a significantly reduced cutin load and striking defects in stomata structure including a lack of cuticular ledges between guard cells ( $\mathrm{Li}$ et al., 2007). These evidences indicate that GPAT4 and GPAT8 are not only involved in cutin formation, but also show that cutin integrity is important in stomatal development. Further analysis indicated that GPATs work together with fatty acyl oxidases in lipid polyester synthesis (Li et al., 2007). Additionally, Long-Chain Acyl-CoA Synthetase 2 (LACS2) protein is also involved in cutin biosynthesis. A lacs2 null mutant displays a reduced thickness of the cutin layer and high cuticle permeability (Schnurr et al., 2004). Lacs2 mutants (sma4 and bre1) have been shown to have enhanced susceptibility to avirulent Pseudomonas syringae and increased resistance to Botrytis cinerea (Tang et al., 2007; Bessire et al., 2007). These phenotypes may be due to more rapid leakage of cell fluids in the substomatal region and an increased release of antifungal compounds through a more permeable cuticle. Mutants of LACS1 (CER8) are deficient in C16 cutin monomers, while C18 cutin monomers are increased (Lu et al., 2009). This indicated that LACS1 is also involved in cutin metabolism. Moreover, a double mutant of lacs1 lacs2 has much lower amounts of both $\mathrm{C} 16$ and $\mathrm{C} 18$ cutin monomers and a highly disorganized cuticle ultrastructure, revealing that LACS1 and LACS2 have overlapping functions in cutin biosynthesis (Lu et al., 2009). Two other proteins, HOTHEAD (Kurdyukov et al., 2006a) and BODYGUARD (Kurdyukov et al., 2006b), have been identified and are proposed to be involved in di-acid formation and cutin assembly, respectively. SHINE1MIN1 is the only known regulatory protein of cutin biosynthesis pathway (Kannangara et al., 2007). 
SHN1MIN1 is a transcription factor belonging to a clade of the AP2-domain/ethylene response element binding protein super family of Arabidopsis (Aharoni et al., 2004; Broun et al., 2004). LACS2 has been shown to be a direct target of SHN1MIN1 (Kannangara et al., 2007).

\subsection{Cuticular wax}

\subsubsection{Chemical profiles of plant cuticular waxes}

Waxes are the second major chemical component of plant cuticles. Waxes have been defined as the lipids that are removed from plant surfaces after brief immersion in an organic solvent, such as chloroform (Post-Beittenmiller, 1996). Wax is predominantly comprised of very-long-chain fatty acids ( $\geq \mathrm{C} 20$ in length) and derived components. Cuticles also contain triterpenoids and minor secondary metabolites, such as sterols and flavonoids (Kunst and Samuels, 2003). However, the composition of wax varies substantially between organ types and species (Mladenova and Stoyanova-Ivanova, 1977; Baker and Hunt, 1979).

Plant cuticular waxes are typically mixtures of free fatty acids, primary and secondary alcohols, aldehydes, alkanes and ketones with chain lengths ranging from 20 to 34 carbons. Alkyl wax esters with chain lengths ranging from $\mathrm{C} 36-\mathrm{C} 70$ have also been identified in waxes (Figure 1B). In the model plant Arabidopsis thaliana, the predominant wax components in stems are C29 alkane, C29 ketone, and C29 secondary alcohols, together with smaller quantities of C30 aldehyde, C26-C30 even chain length primary alcohols, C22-C30 even chain length free fatty acids, and C44-C54 even chain length alkyl wax esters. Alternately, the most abundant cuticular wax 
component in leaves of Arabidopsis is C31 alkane, and leaves lack ketones and secondary alcohols (Jenks et al., 1995).

\subsubsection{Biosynthesis of cuticular wax}

Wax biosynthesis consists of two distinct stages. The first stage is the production of very-long-chain fatty acids (VLCFAs) wax precursors, which is accomplished sequentially in two different cellular compartments: fatty acid synthesis in the plastid to produce $\mathrm{C} 16$ and $\mathrm{C} 18$ fatty acyl precursors and fatty acid elongation in the endoplasmic reticulum to produce C20-C34 VLCFAs. The second stage is modification of monofunctional aliphatics and alkanes. In this latter stage, there are two principal wax biosynthetic pathways: a primary alcohol forming pathway (sometimes called the acyl reduction pathway), which generates primary alcohols and wax esters, and an alkane forming pathway (sometimes called the decarbonylation pathway), which generates aldehydes, alkanes, secondary alcohols and ketones (Figure 3).

Stage 1 Synthesis of VLCFA wax precursors

Saturated VLCFAs with chain lengths between C20 and C34 are the precursors for the biosynthesis of aliphatic cuticular wax components. The formation begins with synthesis of C16 and C18 acyl chains in the plastid (Ohlrogge et al., 1993; Ohlrogge and Browse, 1995). In this process, long carbon chains are assembled through a cycle of four reactions: first, condensation of a $\mathrm{C} 2$ moiety originating from malonyl-acyl carrier protein (ACP) with an acetyl-CoA. After this condensation step, a series of reactions occurs: reduction of the $\beta$-ketoacyl-ACP, dehydration of the $\beta$-hydroxyacyl-ACP, and reduction of the trans- $\Delta^{2}$-enoyl-ACP, resulting in fatty acyl-ACP extended by two carbons longer than the original acetyl molecule. Fatty acid synthesis is catalyzed by 
Figure 3 Proposed pathways for wax biosynthesis in Arabidopsis stems.

Fatty acid synthesis to generate $\mathrm{C} 16$ and $\mathrm{C} 18$ chain lengths occurs in the plastid, and further elongation to $\mathrm{C} \geq 20$ fatty acyl chain lengths occurs in the endoplasmic reticulum. Also, in the ER, very-long-chain fatty acids precursors are modified to generate wax components through two pathways: a primary alcohol forming pathway and an alkane forming pathway.

ACP: acetyl-acyl carrier protein;

FAS: fatty acid synthase complex;

KASI/ KASII/ KASIII: ketoacyl-ACP synthase I/ II/ III;

FATB/FATA: fatty acyl-ACP thioesterase $A$ / fatty acyl-ACP thioesterase $B$;

LACS: long-chain acyl-CoA synthetase;

KCS: $\beta$-keto acyl-CoA synthase;

KCR: $\beta$-ketoacyl reductase;

ECR: enoyl-CoA reductase;

HCD: hydroxyacyl-CoA dehydratase;

VLCFA: very long chain fatty acid;

FAR: fatty acyl-CoA reductase;

WS/DGAT: wax synthase/acyl-CoA diacylglycerol acyl-transferase;

MAH1: mid-chain alkane hydroxylase;

FAE: fatty acid elongase;

CER: ECERIFERUM; 


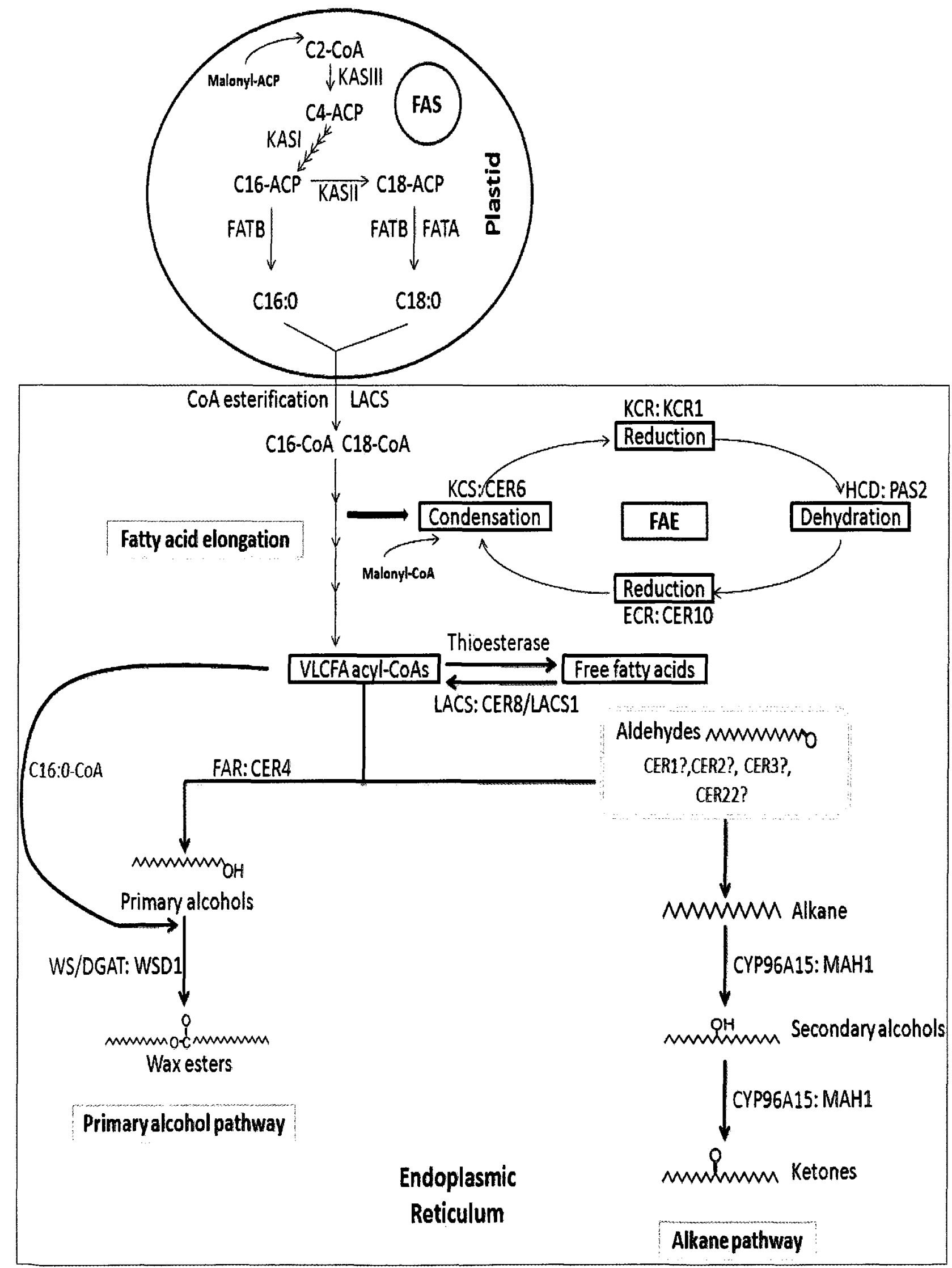


the well characterized soluble fatty acid synthase complexes (FASs), which are localized in the stroma of the plastid (Shimakata and Stumpf, 1982; Stumpf, 1984). These FASs can be divided into three different types based on which ketoacyl-ACP synthase condensing enzyme is present: ketoacyl-ACP synthase III (KASIII) initiates fatty acid biosynthesis using acetyl-CoA as a substrate (Clough et al., 1992); KASI catalyzes C4-C16 elongation, and KASII extends the chain length to C18 (Shimakata and Stumpf, 1982). Chain length specificity of the FAS is thus determined by the condensing enzyme, and the two reductases and dehydratase lack strict specificity and are shared by all three enzyme complexes (Stumpf, 1984).

Once $\mathrm{C} 16$ and $\mathrm{C} 18$ fatty acids are synthesized, they are exported from the plastid to the endoplasmic reticulum (ER) for further elongation. To reach this site, $\mathrm{C} 16$ and C18 acyl chains are liberated from ACP by fatty acyl-ACP thioesterase and then exported out of the plastid by an unknown mechanism. There are two classes of fatty acyl-ACP thioesterases: fatty acyl-ACP thioesterase A (FATA) and fatty acyl-ACP thioesterase B (FATB). The FATA class specifically cleaves C18:0-ACP, while the FATB thioesterases are able to cleave both $\mathrm{C} 16: 0$ and $\mathrm{C} 18: 0$ acyl-chains from acylACP in vitro (Voelker et al., 1996). In Arabidopsis, the fatb mutant has a $20 \%$ and $50 \%$ reduction in total wax load of leaf and stem cuticular wax, respectively, but without significant changes in the distribution of wax composition (Bonaventure et al., 2003). This indicates that a major portion of $\mathrm{C} 16$ and C18 acyl-ACPs are channeled through thioesterase cleavage toward wax biosynthesis (Samuels et al., 2008). Moreover, knockout lines of the FATB gene results in an altered seed morphology, a lower germination rate, reduced growth rate and a reduced level of both palmitate (16:0) and 
stearate (18:0) content in flowers, root, leaves, and seeds. All of these phenotypes indicate that FATB plays an important role in production of saturated fatty acids, which are essential for the generation of cellular membrane components, seed oil production as well as cuticular wax biosynthesis. Free $\mathrm{C} 16$ and $\mathrm{C} 18$ fatty acids become esterified to CoA catalyzed by a long-chain acyl-CoA synthetase (LACS). Nine LACS genes have been identified in Arabidopsis (Shockey et al., 2002). Among these LACS family members, LACS1, LACS2, LACS6, LACS7, and LACS9 have been characterized in detail (Fulda et al., 2002; Schnurr et al., 2002; Schnurr et al., 2004; Tang et al., 2007; Bessire et al., 2007; Lu et al., 2009). CER8/LACS1 (At2g47240) plays a direct role in the modification of VLCFAs, with highest activity for C30 acids (Lu et al., 2009). Mutants of lacs1 have elevated VLCFAs and alkane deficiency of both leaves and stems. The double mutant of lacs1 lacs2 has much lower total wax load and lower amounts of most wax classes than the single mutants, which revealed that LACS1 and LACS2 have overlapping functions associated with the wax biosynthesis in addition to cutin biosynthesis (Lu et al., 2009).

Once the C16:0-CoA and C18:0-CoA are synthesized, they are translocated to the ER for further elongation. This process is catalyzed by fatty acid elongases (FAE) (von Wettstein-Knowles, 1982), multienzyme complexes that are located in the ER (Xu et al., 2002; Zheng et al., 2005; Joubes et al., 2008). Similar to fatty acid synthesis in the plastid, elongation of long-chain acids (C16, C18) to VLCFAs involves a 2-carbon extension cycle. The $\mathrm{C} 2$ units for FAE are malonyl-CoA, rather than malonyl-ACP. The four consecutive enzymatic reactions are: condensation of malonyl-CoA with fatty acylCoA, reduction of the resulting $\beta$-ketoacyl-CoA, dehydration of $\beta$-hydroxyacyl-CoA and 
reduction of trans- $\Delta^{2}$-enoyl-CoA (Fehling and Mukherjee, 1991). Several elongation cycles are needed to synthesize $\mathrm{C} 20-\mathrm{C} 34$ acyl chains for further production of aliphatic wax components. The entire elongation process is carried out by several distinct FAE complexes with different chain length specificities (von Wettstein-Knowles, 1993). The substrate specificity of the FAE complex is largely determined by the $\beta$-ketoacyl-CoA synthase (KCS), which catalyzes the first step of the cycle (Millar and Kunst, 1997). In the Arabidopsis genome, a large family of $21-K C S$ like sequences have been identified (Lechelt-Kunze et al., 2003; Dunn et al., 2004; Blacklock and Jaworski, 2006; Joubes et al., 2008). Among all the condensing enzymes, CER6 is thus far the only wax-specific KCS and it is involved in elongation of fatty acyl-CoA longer than C22 (Millar et al., 1999; Fiebig et al., 2000; Hooker et al., 2002). Disruption of the CER6 gene results in only a $19 \%$ total wax load compared to wild-type levels and the mutant is completely devoid of epicuticular wax crystals (Millar et al., 1999). In addition, cer6 mutants are male-sterile indicating that reduced levels of cuticular wax influences stigma-pollen interaction (Millar et al., 1999; Fiebig et al., 2000). Other KCS genes in Arabidopsis, KCS1 and FIDDLEHEAD, are upregulated in Arabidopsis stem epidermal cells and likely have roles in fatty acid elongation in epidermal cells for wax production (Todd et al., 1999; Yephremov et al., 1999; Suh et al., 2005). Maize GLOSSY8 (GL8) is found to be a $\beta$ keto acyl reductase $(K C R)$ that catalyzes the reduction of $\beta$-ketoacyl-CoAs during VLCFA synthesis (Xu et al., 1997). In the Arabidopsis genome, two putative KCRs, AtKCR1 and AtKCR2, were identified and both of them are expressed at high levels in the stem epidermis (Suh et al., 2005; Beaudoin et al., 2009). A knockout of AtKCR1 is embryo lethal, while partial suppression of AtKCR1 activity results in reduced cuticular 
wax load in addition to affecting VLCFA composition in sphingolipids, seed triacylglycerols, and root glycerolipids. The role of AtKCR2 is unknown. Arabidopsis PASTICCINO2 (PAS2) encodes the fatty acyl-CoA dehydratase, which is limiting for VLCFA synthesis and essential for plant development (Bath et al., 2008). In the pas2 mutant, lipid fractions of Arabidopsis that contain VLCFAs were perturbed, while complete loss of PAS2 function causes embryo lethality. In Arabidopsis, CER10 was identified as an enoyl-CoA reductase (ECR) that is required for the synthesis of all the VLCFA-containing lipids (Zheng et al., 2005). cer10 mutants exhibit reduced organ size, have $40 \%$ of the cuticular wax load of wild-type, and a reduction of VLCFAs in seed triacylglycerols and sphingolipids (Zheng et al., 2005). Interestingly, knockout mutants of CER10 are not lethal and still accumulate some VLCFAs and cuticular waxes. This indicates that unknown enzyme(s) function in a partially redundant manner with CER10/ECR (Zheng et al., 2005).

Stage 2 Synthesis of wax compounds

Once the VLCFAs are generated in the ER, two main wax biosynthetic pathways generate the different aliphatic wax components (Samuels et al., 2008).

The primary alcohol pathway is also called the acyl reduction pathway (Kunst et al., 2003). Biochemical studies suggested that generation of primary alcohols can occur via a two-step reaction carried out by two independent enzymes: an NADH-dependent acylCOA reductase and an NADPH-dependent aldehyde reductase, such that the aldehyde intermediate can be isolated (Kolattukudy, 1971). Later studies indicated that the reduction of VLCFAs to alcohols can alternatively be carried out by single alcoholforming FAR enzymes (Kolattukudy, 1970; Vioque and Kolattukudy, 1997; Metz et al., 
2000). In the Arabidopsis genome, eight FAR-like genes encoding enzymes of this latter type have been identified and one of them is CER4 (Rowland et al., 2006). CER4 is the major FAR responsible for primary alcohol formation in Arabidopsis cuticular wax (Hannoufa et al., 1993; Jenks et al., 1995; Rowland et al., 2006). Although much of the primary alcohols are secreted to the cuticle in free form, some of them become esterified to acyl groups through the action of a wax synthase (WS) enzyme (Lai et al., 2007). Recently, one gene, WSD1, from Arabidopsis has been found to be important for cuticular wax ester formation in stems ( $\mathrm{Li}$ et al., 2008). This gene is a putative bifunctional enzyme, which has high WS activity and substantially lower acylCoA:diacylglycerol acyltransferase activity (Li et al., 2008).

The alkane-forming pathway is also called the decarbonylation pathway. A decarbonylation reaction was first proposed by Kolattukudy et al (1972) to be part of this pathway. Later biochemical experiments provided support for aldehydes as intermediates leading to formation of alkanes (Kolattukudy et al., 1974). A decarbonylation reaction releases one carbon monoxide (CO) from aldehydes to generate odd chain length alkanes (Cheesbrough and Kolattukudy, 1984; SchneiderBelhaddad and Kolattukudy, 2000). However, the enzymes responsible for aldehyde and alkane formation remain elusive and chemical mechanisms other than decarbonylation are possible. Radioactive labeling studies and genetic evidence indicated that secondary alcohols and ketones were generated by hydroxylation and oxidation, respectively (Kolattududy and Liu, 1970; Khan and Kolattukudy, 1974). In a reverse genetics study, a mid-chain alkane hydroxylase (MAH1) responsible for these reactions was identified in Arabidopsis (Greer et al., 2007). MAH1 encodes a 
cytochrome P450-dependent enzyme (CYP96A15) and is responsible for secondary alcohol and ketone formation in cuticular wax of Arabidopsis stems.

\subsubsection{Regulation of wax biosynthesis}

Resistance of plants to environmental stresses, microbial pathogens and insects are linked to wax accumulation and wax composition (Post-Beittenmiller, 1996). However, knowledge about the regulatory mechanisms governing wax production is currently limited.

The influence of environmental stresses on the expression of two genes encoding wax biosynthetic enzymes in Arabidopsis, CER2 and CER6, has been investigated (Xia et al., 1997; Hooker et al., 2002). CER6 transcription is induced by light and osmotic stress, while CER2 expression is rather influenced by cytokinin hormone (Xia et al., 1997; Hooker et al., 2002).

Currently two transcription factors, SHN1MIN1 and MYB30, have been reported to be involved in wax biosynthesis (Broun et al., 2004; Aharoni et al., 2004; Raffaele et al., 2008). Overexpression of WIN1/SHN1 results in an increased of wax load on both leaves and stems and also the induction of some wax genes, such as CER1, CER2, and KCS1 (Aharoni et al., 2004; Broun et al., 2004). Additionally, WIN1/SHN1 can activate genes encoding cutin biosynthetic enzymes, such as LACS2 and GPAT4, (Kannagara et al., 2007). MYB30 knockout and overexpression lines display alterations in leaf epidermal wax components and DNA microarray analysis of overexpressing MYB30 lines show that fatty acid elongase (FAE) components are putative target genes of MYB30 (Raffaele et al., 2008). Additionally, the MYB41 gene, which encodes an R2R3-MYB transcription factor, has been suggested to be involved in cuticle 
biosynthesis based on the fact that over-expression of AtMYB41 led to higher rates of water loss, faster chlorophyll leaching in ethanol, and a patchy toluidine-blue staining phenotype (Cominelli et al., 2007).

CER7, a subunit of the exosome, is also important for wax biosynthesis. The exosome is involved in RNA processing and degradation (Hooker et al., 2007). An mRNA encoding a CER3 repressor was postulated to be the target of CER7 ribonuclease in epidermal cells (Hooker et al., 2007). Therefore, specific degradation of the repressor's mRNA by CER7 may allow CER3 expression and the alkane forming pathway to proceed (Hooker et al., 2007).

\subsubsection{Transportation of cuticular wax components}

The transport of cuticular waxes can be divided into three stages. First, the wax constituents generated in the endoplasmic reticulum (ER) are delivered to the plasma membrane (PM). Second, all the wax components are released from the PM into the cell wall. Third, the wax components are transported through the cell wall and to the cuticle at the surface.

A number of wax biosynthetic enzymes have been found to be associated with the ER using green fluorescent protein (GFP) as a tag: these are CER6, CER10, CER4, MAH1, and WSD1 (Millar et al., 1999; Fiebig et al., 2000; Zheng et al., 2005; Rowland et al., 2006; Greer et al., 2007; Li et al., 2008). These evidences indicate that both the intermediate metabolites and the final products of the wax biosynthesis pathway are produced in the ER. However, the mechanisms of exportation of wax components from the ER to PM are unknown. Two models have been proposed by analogy with other lipid transport processes (Kunst and Samuels, 2003; Schulz and Frommer, 2004). One 
possibility is that wax components are directly transferred to the PM at ER-PM sites of contact. The second model suggests that wax molecules are transported by vesicles moving along the secretory pathway from ER to Golgi and then to the PM.

When wax components reach the PM, they must be exported to the apoplastic environment. Two ATP binding cassette (ABC) transporters, CER5 and WBC11, have been shown to be important for this process (Pighin et al., 2004; Bird et al., 2007; Panikashvili et al., 2007; Ukitsu et al., 2007). Both CER5 and WBC11 ABC transporters are localized to the PM, and cer5 and wbc11 mutants accumulate wax molecules inside the epidermal cells (Pighin et al., 2004; Bird et al., 2007; Panikashvili et al., 2007; Ukitsu et al., 2007). Since there is no direct evidence that CER5 and WBC11 can interact with wax molecules as substrates, the details on how these two transporters transport wax molecules across the lipid bilayer remains to be investigated. Moreover, the double mutant cer5 wbc11 still accumulats wax in stems to a low level (Bird et al., 2007). This suggests that additional transporters are involved in this process.

After reaching the cell wall, wax components are further secreted across the hydrophilic cell wall layer to accumulate and crystallize at the surface. Wax molecules are highly lipophilic and have very low solubility in the cell wall environment. It is possible that this transport process is carried out by special structures that help guide the wax molecules to the cuticle, but evidence for this is lacking. Lipid transfer proteins (LTPs) have also been suggested as being involved in shuttling waxes through the cell wall. LTPs are very small proteins of a large superfamily that can diffuse and move through the pectin matrix of the primary cell wall (Kader, 1996; Arondel et al., 2000; Rogers and Bankaitis, 2000). LTPs are highly expressed in the epidermis and secreted 
into the apoplast (Thoma et al., 1993; Pyee et al., 1994). Additionally, the structures of LTPs revealed that they contain a flexible hydrophobic pocket which can bind to linear acyl lipids (Bouliez et al., 2000). A glycosylphosphatidylinositol-anchored lipid transfer protein (LTPG) involved in cuticle development was identified in a reverse genetic screen (Debono et al., 2009). It is highly expressed in the epidermis of Arabidopsis stems during cuticle biosynthesis. LTPG is localized in the plasma membrane on the cell wall side, where wax is actively secreted (Lee et al., 2009). Disruption of the LTPG gene resulted in an alteration of cuticular lipid composition, and a more diffuse cuticular layer structure (Debono et al., 2009). All these data indicate that LTPG is involved either directly or indirectly in cuticular lipid deposition and may function as a component of the cuticular lipid export machinery (Debono et al., 2009; Lee et al., 2009).

\subsection{Objective of project}

The purpose of my thesis project was to identify and characterize novel cer mutants and to characterize the uncloned CER9 gene. The epicuticular wax crystal structures and cuticular wax compositions of a set of novel wax deficient mutants were compared to a set of previously identified CER mutants, in order to predict allelism between the sets of mutants. I further phenotypically characterized two potentially novel mutants called line8 and line85. I mapped the two loci to specific chromosome locations and tested for allelism with other cer mutant loci mapping to the same region, and found that LINE8 and LINE85 were in fact novel alleles of CER6 and CER19, respectively. Finally, I used a map-based cloning approach to localize the position of CER9 on chromosome IV, and did further phenotypic characterization of the cer9 mutant to gain insights into the function of CER9 in cuticular wax biosynthesis. 


\section{Chapter 2 Identification of Novel Eceriferum Mutants}

2.1 Introduction: eceriferum (cer) mutants in Arabidopsis thaliana

Arabidopsis mutants with defects in the production of epicuticular wax are usually called eceriferum (cer) mutants (Koornneef et al., 1989). These mutants can be easily identified visually because of their brighter green stems and siliques compared to wildtype plants. Studies of wax-deficient mutants in other plant species have been performed, including Brassica oleracea (Baker, 1974; Macey, 1974), Brassica napus (Holloway et al., 1977), Zea maize (Bianchi, 1979), Hordeum vulgare (von WettsteinKnowles, 1979), and Pisum sativum (Macey and Barber, 1970).

To date, many wax-deficient loci have been identified in Arabidopsis including 24 CER loci, FATB, KCS1, WSD1, MAH1, and LTPG (Koornneef et al., 1989; McNevin et al., 1993; Rashotte et al., 2004; Bonaventure et al., 2003; Todd et al., 1999; Li et al., 2008; Greer et al., 2007; Debono et al., 2009) (Table 1). Koornneef et al. (1989) identified 89 cer mutants in Arabidopsis that mapped to 21 different loci (CER1 to CER20 and TRANSPARENT TESTA5) by screening for plants with glossy stems. A CER21 locus was reported (McNevin et al., 1993), but later revealed to be allelic to CER3 (Rowland et al., 2007). Rashotte et al. (2004) conducted a non-visual screen using screening by gas chromatography and identified three novel genes that have been designated CER22, CER23, and CER24. In Arabidopsis, CER1-CER8, CER10, FATB, KCS1, WSD1, MAH1, and LTPG have been cloned so far (Aarts et al., 1995; Negruk et al., 1996; Fiebig et al., 2000; Chen et al., 2003; Bonaventure et al., 2003;

Pighin et al., 2004; Zheng et al., 2005; Rowland et al., 2006; Rowland et al., 2007; 
Hooker et al., 2007; Greer et al., 2007; Li et al., 2008; Debono et al., 2009; Lu et al., 2009) (Figure 4). The biosynthetic or transport activities of these protein products were described in Chapter 1 and summarized here in Table 1 and Figure 5.

In Arabidopsis, the genes disrupted in the cer mutant collection are involved in wax biosynthesis, wax intra- or extra-cellular transportation, or regulation. The cer loss-offunction mutants generally exhibit changes in the wax crystalline structures and in some cases the wax crystals are absent. These structures are typically observed by scanning electron microscopy (SEM). The major wax crystalline structures observed on plant surfaces are platelets, tubules, threads, transverse rodlets, dendrites, and longitudinal rodlets (Jeffree, 2006). On Arabidopsis stems the wax structures are predominated by longitudinal bundles of rodlets. There are no epicuticular wax crystals on wild-type Arabidopsis leaves. Studies indicate that the epicuticular wax crystalline structures are largely determined by the composition of wax components (Jeffree, 2006). Epicuticular wax that has a single predominating component usually has a characteristic structural type. For example, the primary alcohol-rich waxes have plate-shaped crystals, while wax rich in $\beta$-diketones have a tubular morphology (Jeffree, 2006). However, the correlation between wax composition and crystalline structure is not very strict, especially in complex mixtures of cuticular waxes.

The visual screening method can only identify mutants with a sufficiently low density of crystals that would give a detectable glossy stem phenotype due to a difference in light scattering. Mutants having an altered chemical composition that does not lead to changed visual reflectance are not detected in visual screens. For example, MAH1 is critical for secondary alcohol and ketone formation in Arabidopsis stems, but 
mah1 mutant still has a glaucous wild-type stem phenotype (Greer et al., 2007). A more rigorous method to screen wax-deficient mutants using gas chromatography (GC) analysis can more sensitively detect alterations in total wax load and/or cuticular wax constituents (Rashotte et al., 2004).

The objective of this chapter was to characterize a set of unpublished wax deficient mutants identified by the Rowland lab. These were temporally termed 'line' mutants until allelism with existing cer mutants could be established. It was anticipated that some of these line mutants would represent novel cer loci, as the forward screen to identify the original set of cer mutants was not done to saturation (Koorneef et al., 1989). The stem wax crystal morphology and stem wax composition of ten line mutants and 11 cer were characterized by SEM and GC, respectively, to place them into phenotypically similar groups. Genetic complementation crosses were then performed to test for allelism within these groups. 


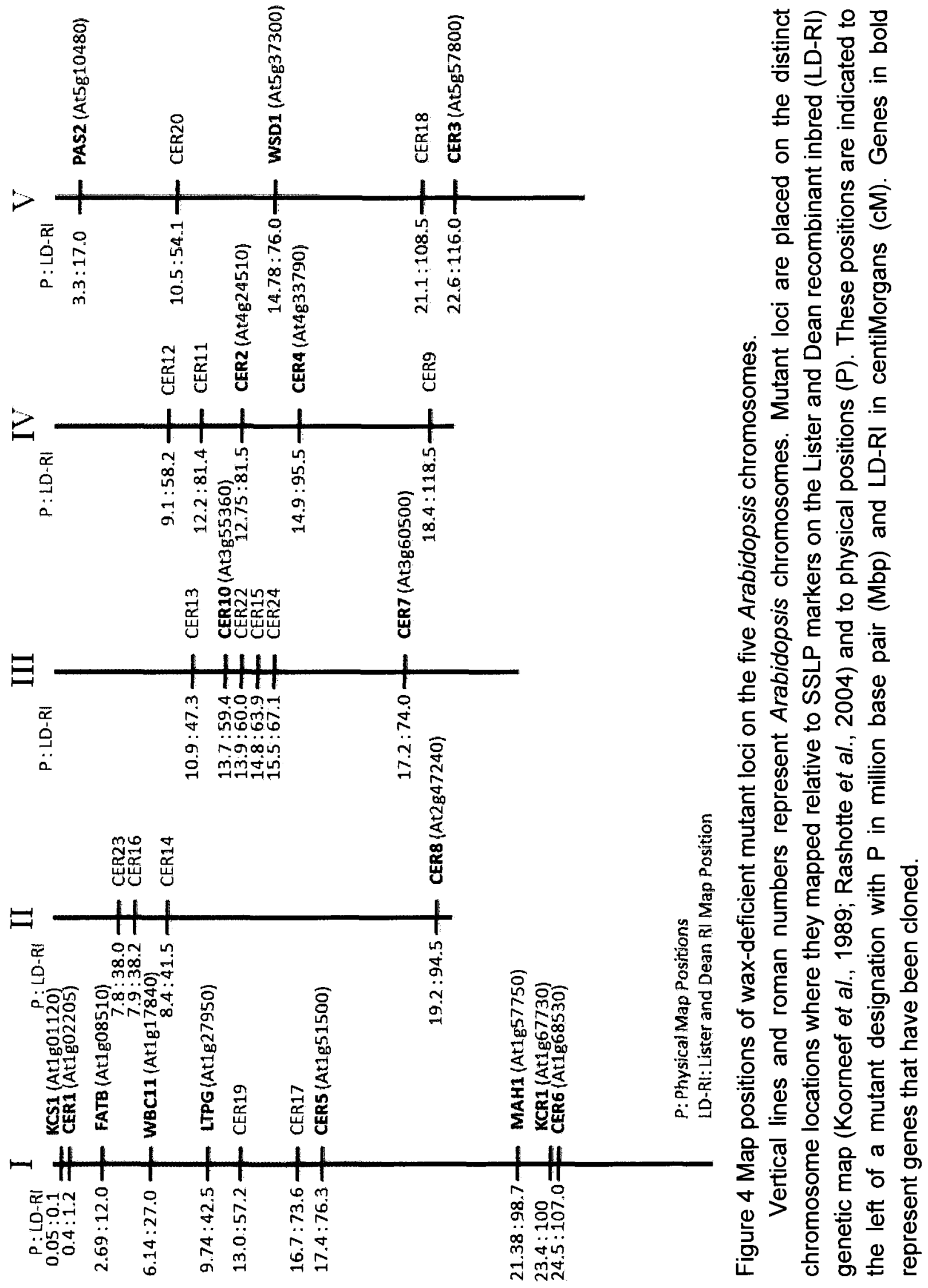




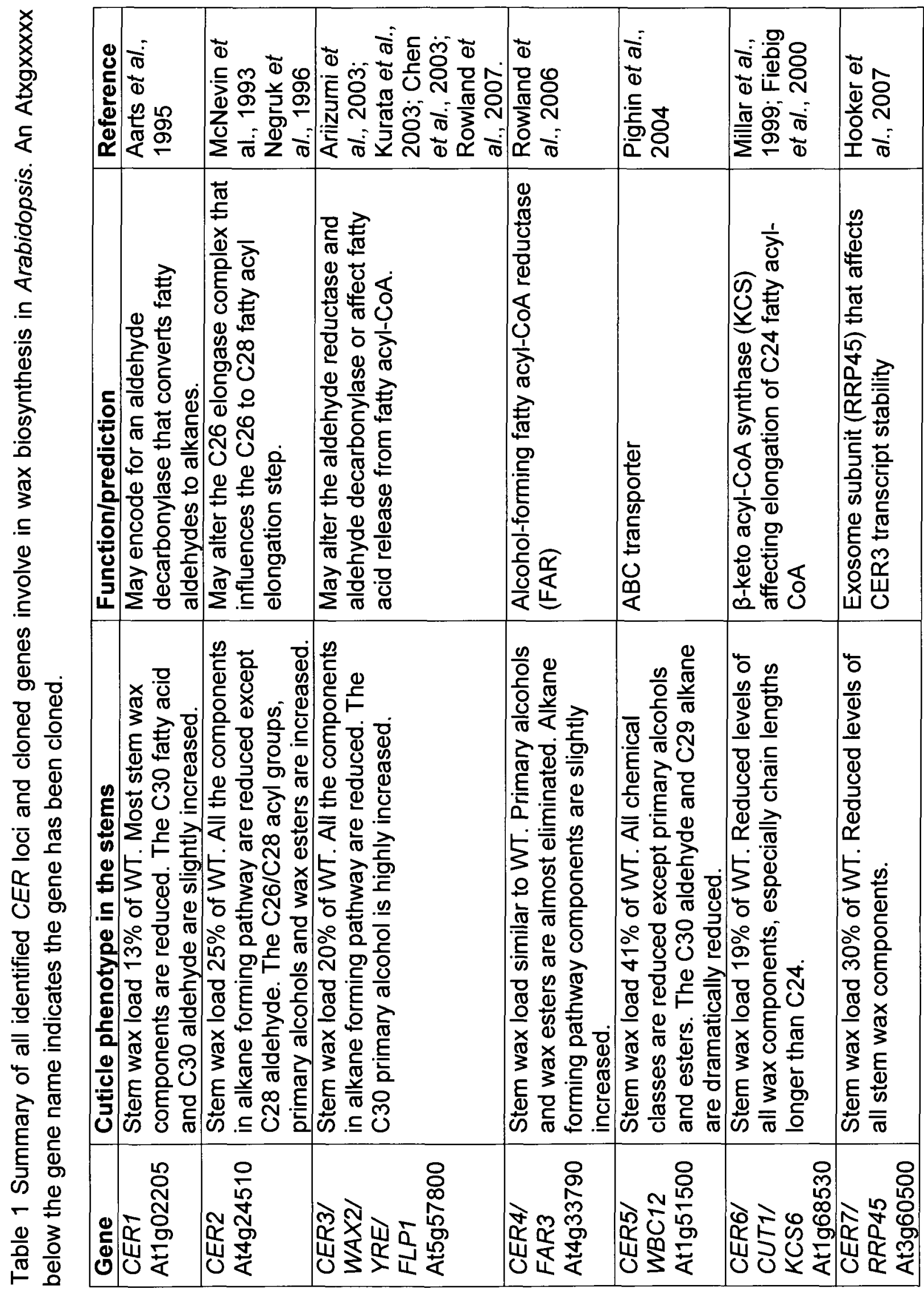




\begin{tabular}{|c|c|c|c|c|c|c|c|c|c|}
\hline 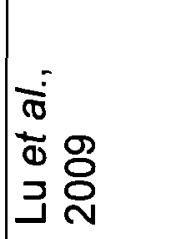 & 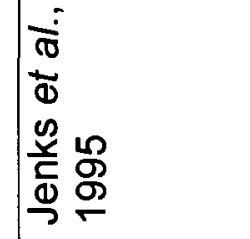 & 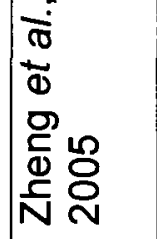 & 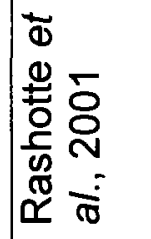 & 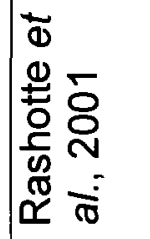 & 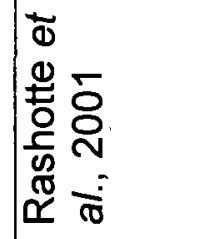 & 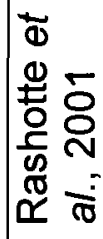 & 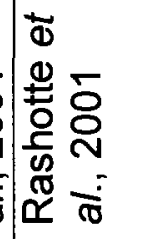 & 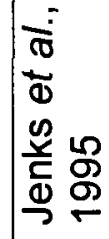 & 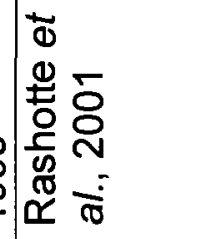 \\
\hline 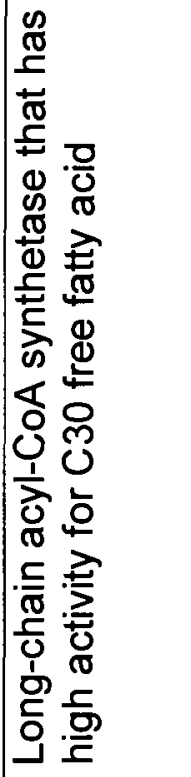 & 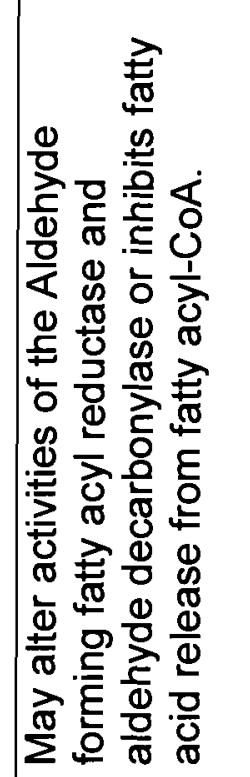 & 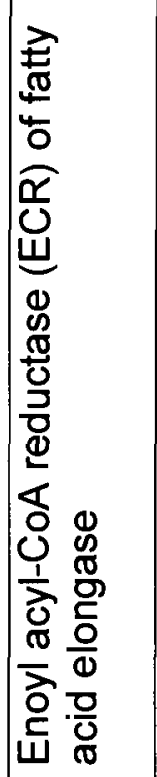 & 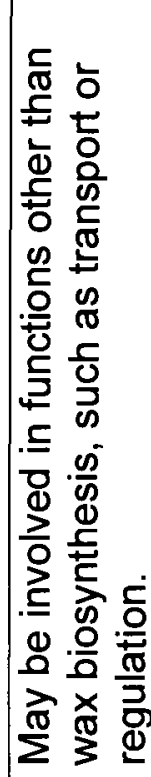 & 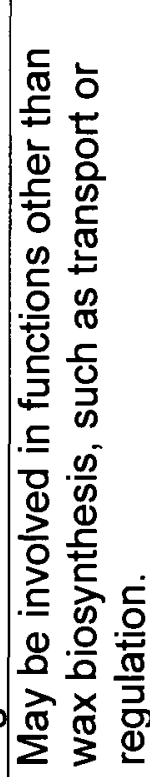 & 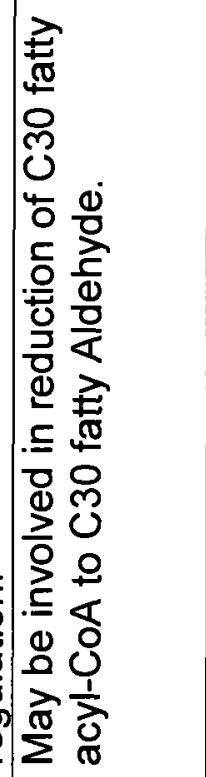 & 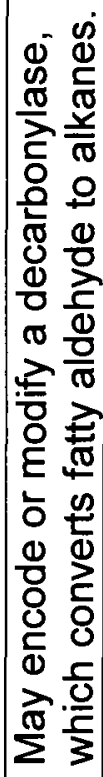 & 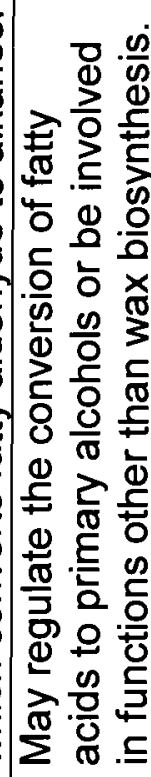 & 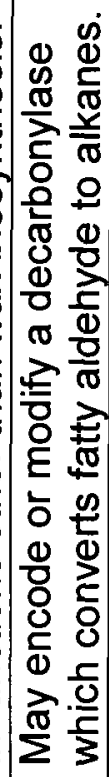 & 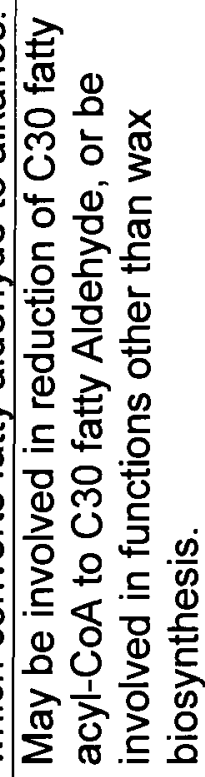 \\
\hline 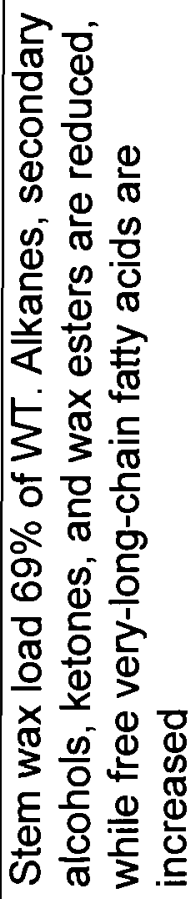 & 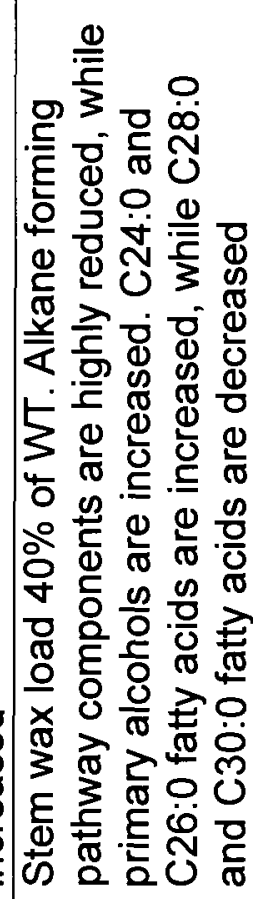 & 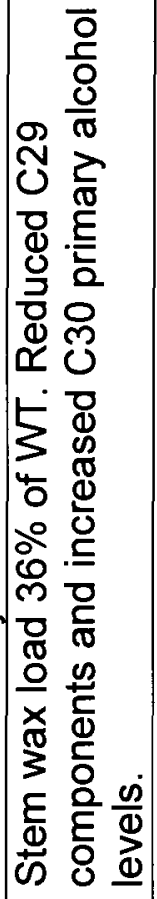 & 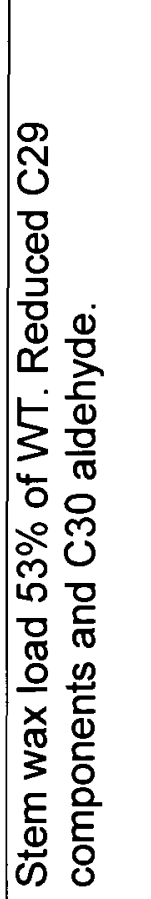 & 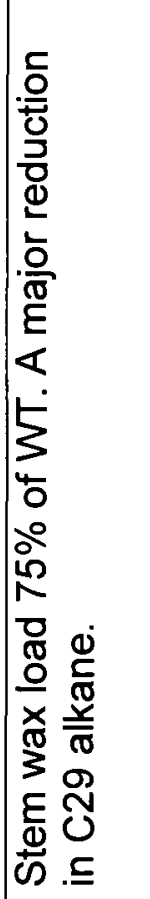 & 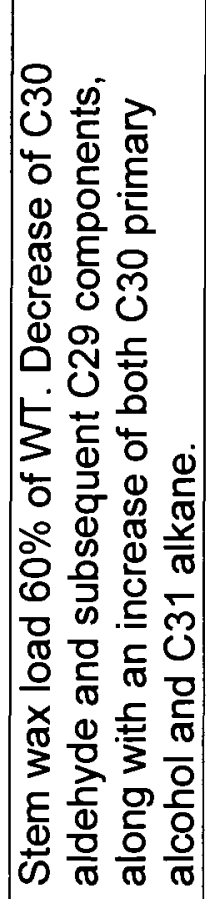 & 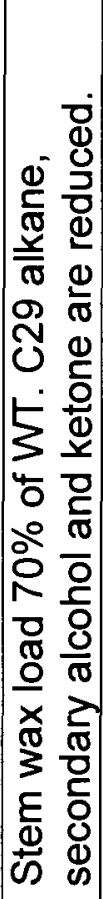 & 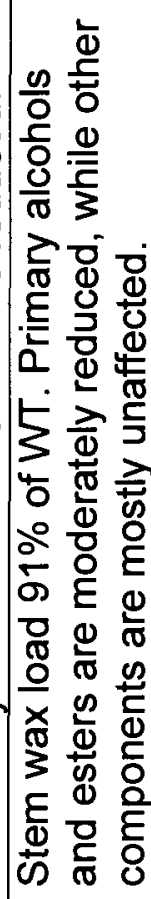 & 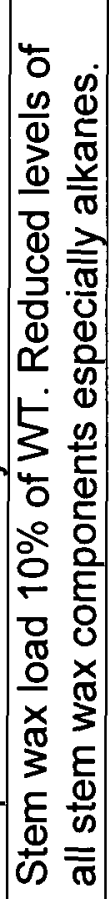 & 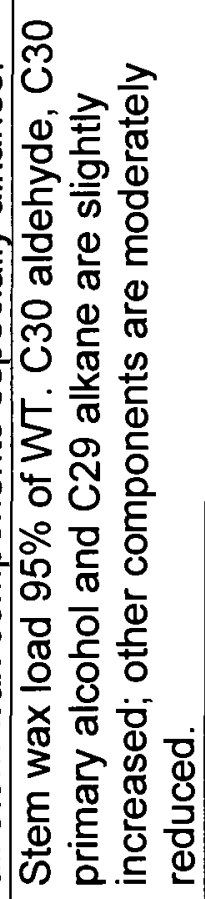 \\
\hline 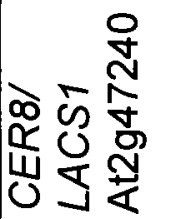 & $\frac{9}{\frac{1}{0}}$ & 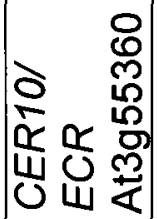 & & $\frac{N}{\frac{N}{\alpha}}$ & & $\frac{⿱ 亠}{\frac{\pi}{\alpha}}$ & $\frac{n}{\frac{10}{\alpha}}$ & $\frac{6}{\frac{1}{4}}$ & $\frac{N}{\frac{1}{\alpha}}$ \\
\hline
\end{tabular}




\begin{tabular}{|c|c|c|c|c|c|c|c|c|c|}
\hline 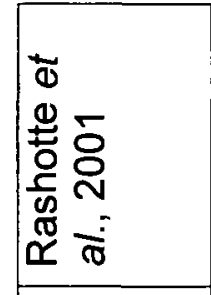 & 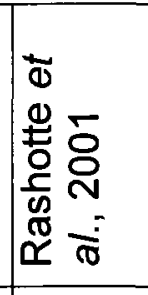 & 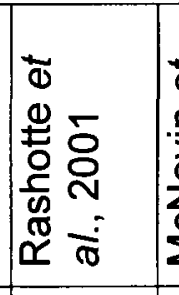 & 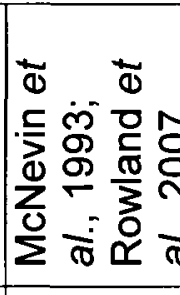 & 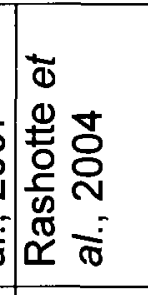 & 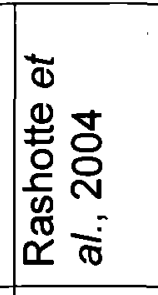 & 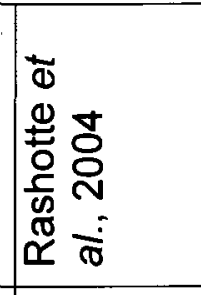 & 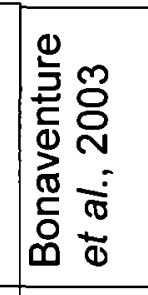 & 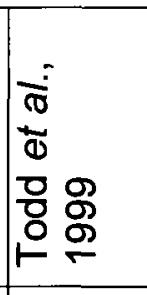 & 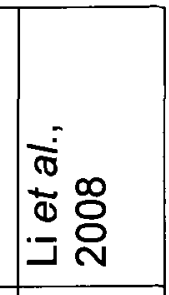 \\
\hline 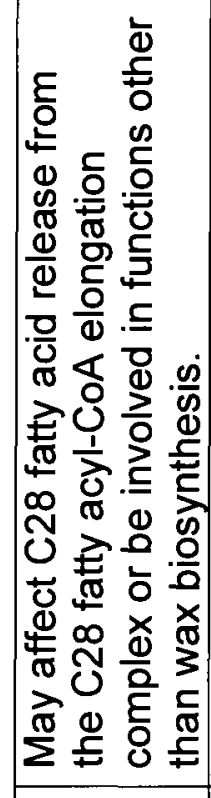 & 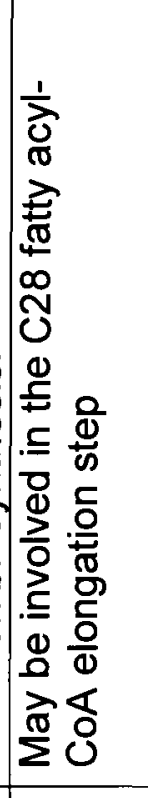 & 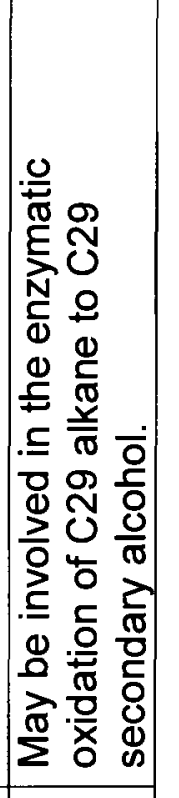 & & 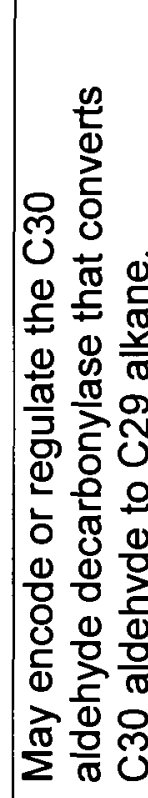 & 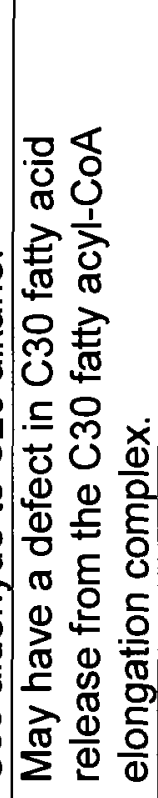 & 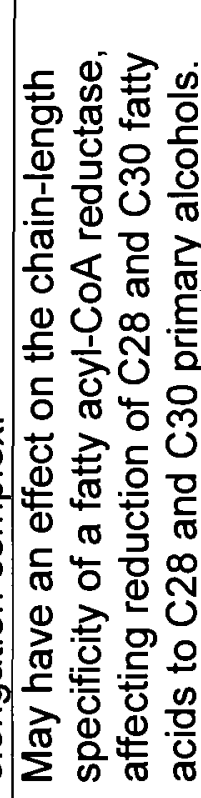 & 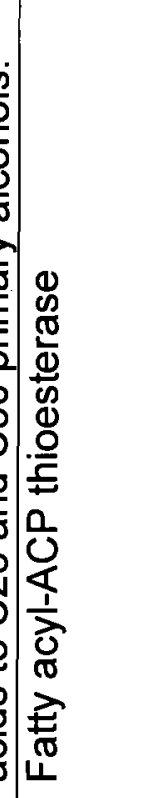 & 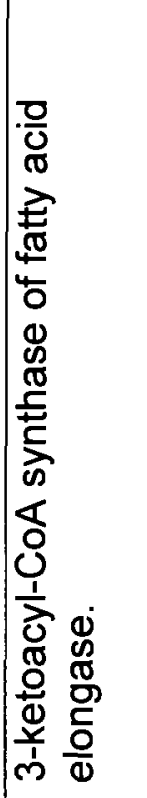 & 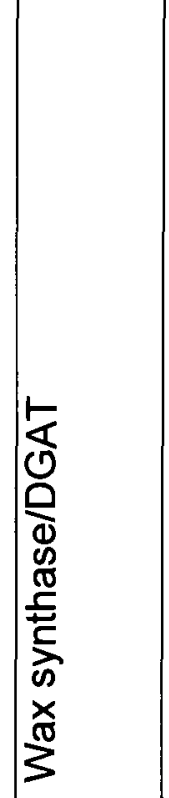 \\
\hline 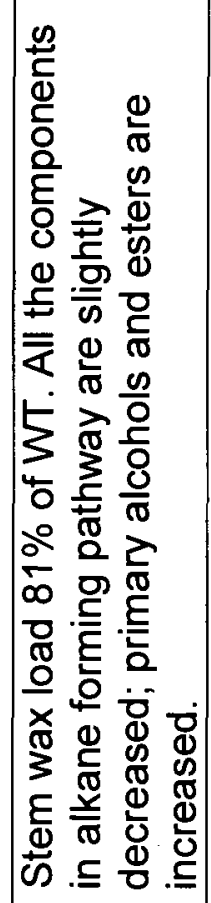 & 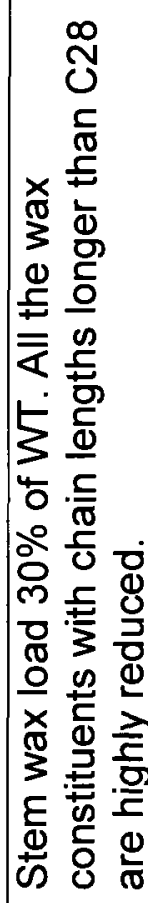 & 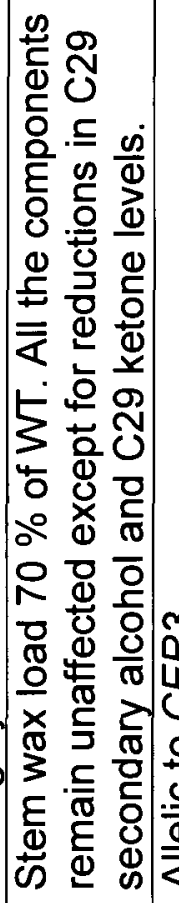 & & 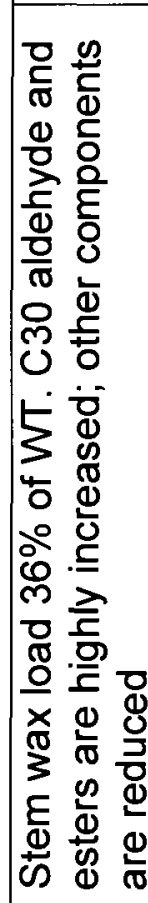 & 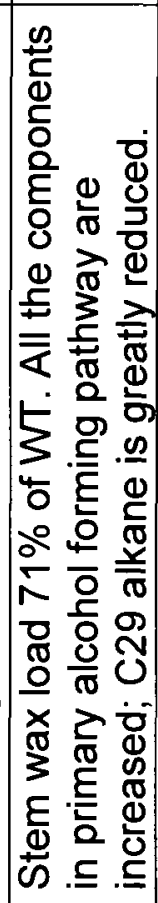 & 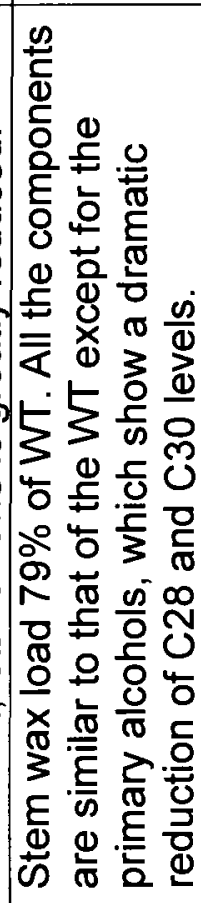 & 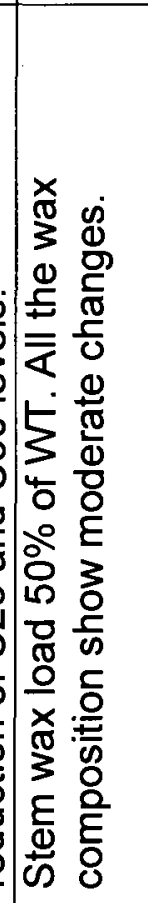 & 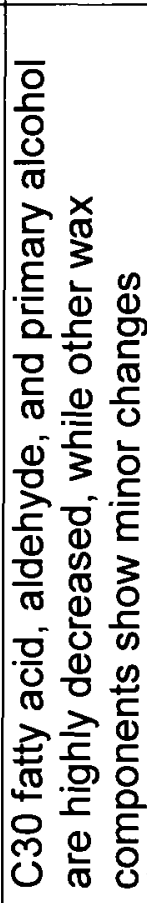 & 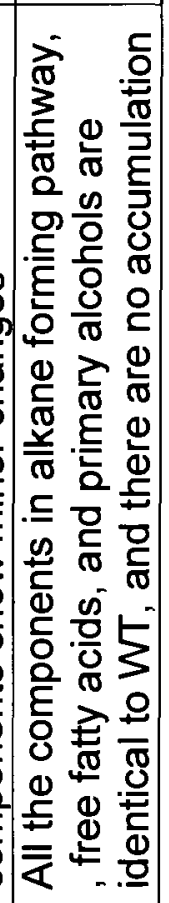 \\
\hline & 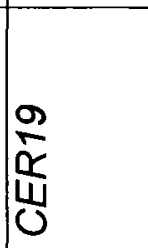 & 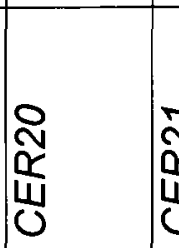 & & & & jo & 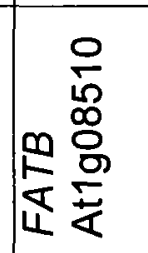 & 总 & 旡 \\
\hline
\end{tabular}




\begin{tabular}{|c|c|c|c|c|c|}
\hline & 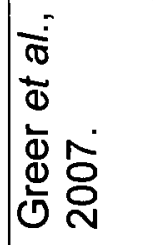 & 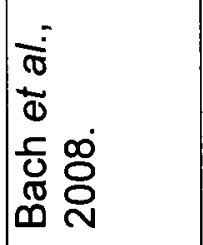 & 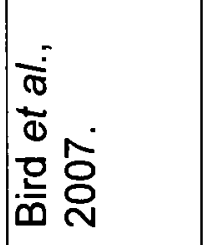 & 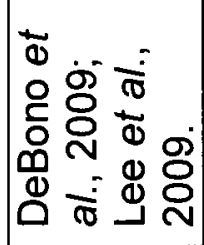 & 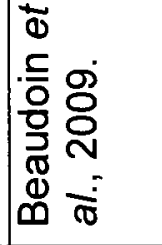 \\
\hline & 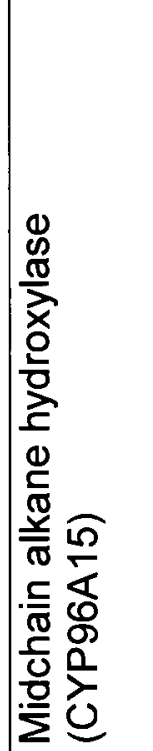 & 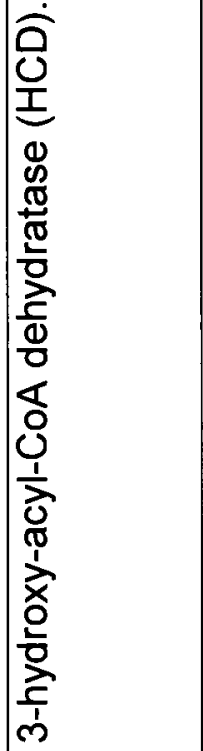 & 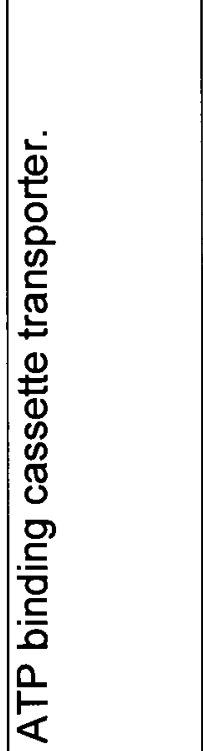 & 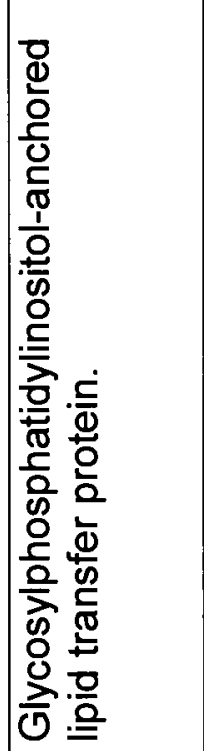 & 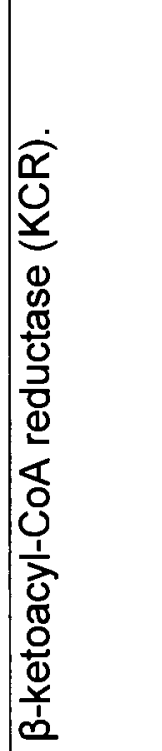 \\
\hline & 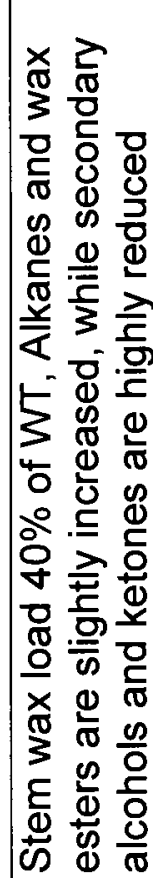 & 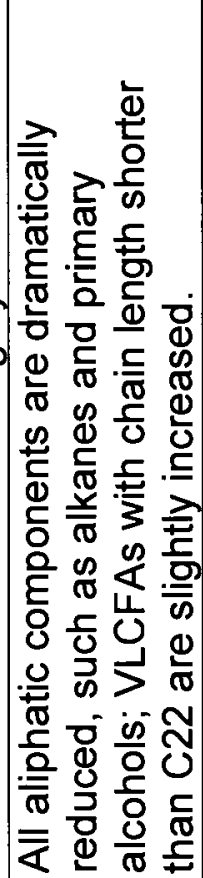 & 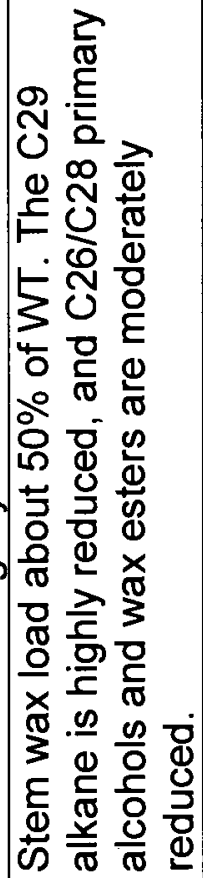 & 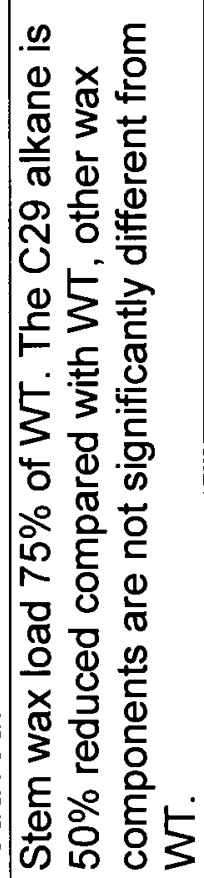 & 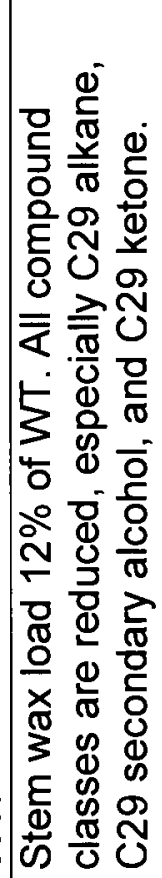 \\
\hline & 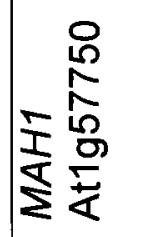 & 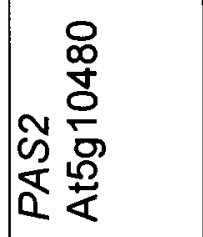 & 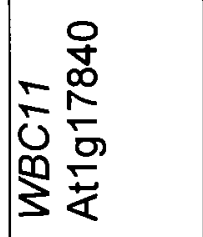 & 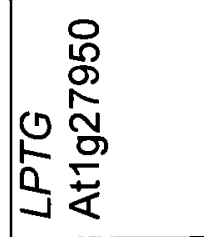 & 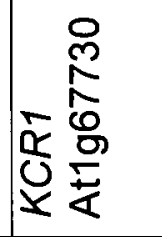 \\
\hline
\end{tabular}




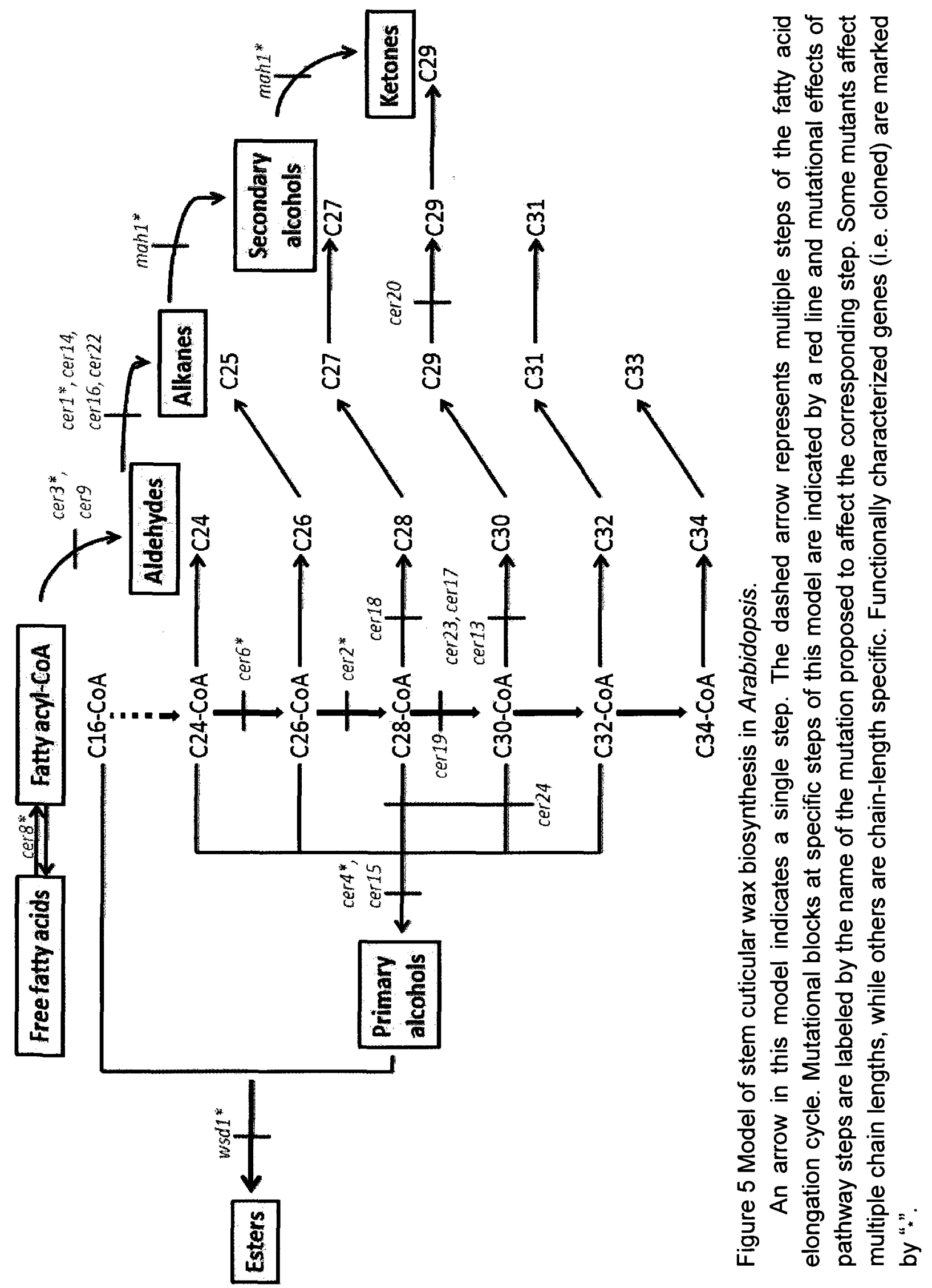




\subsection{Materials and Methods}

\subsubsection{Plant materials and growth conditions}

Two wild-type ecotypes of Arabidopsis thaliana were used: Columbia-0 (Col-0) and Landsberg erecta (Ler), which were kindly provided by Professor Ljerka Kunst of the Department of Botany, University of British Columbia. Eceriferum (cer) mutants were obtained from Professor Ljerka Kunst who obtained them from ABRC (Arabidopsis Biological Resource Centre). All the line mutants were identified by Professor Owen Rowland.

Seeds were sown onto AT plates. AT medium contents: $50 \mathrm{mM}$

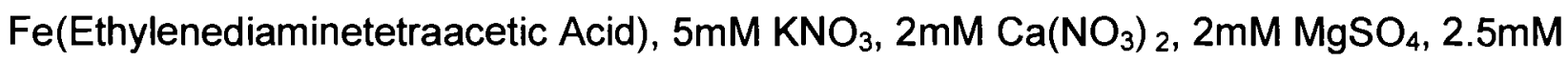
$\mathrm{KH}_{2} \mathrm{PO}_{4}, 1 \mathrm{~mL} / \mathrm{L}$ micronutrients (stock: $70 \mathrm{mM} \mathrm{H} \mathrm{H}_{3} \mathrm{BO}_{3}, 15 \mathrm{mM} \mathrm{MnCl}_{2} \times 2 \mathrm{H}_{2} \mathrm{O}, 0.5 \mathrm{mM}$ $\mathrm{CuSO}_{4}, 1 \mathrm{mM} \mathrm{ZnSO}_{4} \times 7 \mathrm{H}_{2} \mathrm{O}, 0.2 \mathrm{mM} \mathrm{NaMoO}_{4} \times 2 \mathrm{H}_{2} \mathrm{O}, 10 \mathrm{mM} \mathrm{NaCl}, 0.01 \mathrm{mM} \mathrm{CoCl}_{2} \times 6 \mathrm{H}_{2} \mathrm{O}$ ) and $7 \mathrm{~g} / \mathrm{L}$ agar (BioShop, Burlington ON). Plates containing sown seeds were incubated at $4^{\circ} \mathrm{C}$ for 3-4 days to break dormancy and to give synchronous seed germination. The plates were then moved to a tissue culture cabinet with continuous light and at $22^{\circ} \mathrm{C}$ temperature for 1-2 weeks. The seedlings were then transferred to soil. Soil was purchased from Berger Peat Moss-Le Group Berger Itee (Saint-Modestem, QC). The soil was soaked with water and autoclaved before using. $45 \mathrm{~mL}$ of $1 \mathrm{~g} / \mathrm{L} 20-20-20$ all purpose fertilizer Plant-Pod ${ }^{\circledR}$ (Plant Product Co. Ltd, Brampton ON) was added to each square pot $(9 \mathrm{~cm}$ by $9 \mathrm{~cm})$. Plants were grown in an environment-controlled growth chamber (Conviron, Controlled Environment Ltd, Winnipeg, MB) with a long-day photoperiod (18 hours light and 6 hours dark) and at $22^{\circ} \mathrm{C}$.

\subsubsection{Scanning electron microscopy}


Scanning electron microscopy (SEM) was used to examine the densities and shapes of epicuticular wax crystals of each mutant and wild-type plant. One $\mathrm{cm}$ stem fragments taken from the middle of the plant inflourescence were cut from 6 week-old plants and placed on an SEM stub, which had been affixed with a double-sided sticky tab. The SEM sample platform was then placed in a sealed plastic container containing a layer of drierite (W.A.Hammond Drierite). Samples were dried for 3-4 days, and then coated with $15 \mathrm{~nm}$ of gold using a gold sputter coater (Anatech Hummer). The surfaces were subsequently imaged on a JSM-6400 scanning electron microscope (JEOL) located at the Nanoprobe (SEM) facility of Carleton University.

\subsubsection{Gas chromatography}

Gas chromatography (GC) was performed to determine the total wax load and chemical composition of stems and leaves of each mutant. All stem samples were isolated from six week-old plants and rosette leaf samples were taken from 5-6 weekold plants.

A digital caliper was used to measure the diameter of the stem, taken at 3 positions: the top, the middle, and the bottom of the selected segment and the average was then used to calculate the surface area of the stem. The length of stem used was typically $9 \mathrm{~cm}-12 \mathrm{~cm}$. For each repeat, $2-3$ stem segments of similar lengths were used in order to ensure a sufficient amount of wax was extracted for GC analysis. Three duplicates of each mutant and wild-type were prepared. For leaves, each repeat contained at least five rosette leaves and three duplicates were prepared.

All the GC extraction tubes were rinsed with HPLC-grade chloroform three times to eliminate contaminants. $10 \mu \mathrm{l}$ of $0.1 \mathrm{mg} / \mathrm{ml}$ internal standard: methyl heptadecanoate 
(17:1), was added to each $G C$ vial by using a $10 \mu l$ Hamilton syringe. Each tube was then filled up with HPLC-grade chloroform. One extra GC extraction tube containing no wax sample was prepared and used as a blank control.

The stem segments were dipped together in HPLC-grade chloroform for exactly 30 seconds. Leaves were dipped in HPLC-grade chloroform one at a time for exactly 30 seconds, and then the leaves were wrapped in plastic wrap maintaining the shape of each rosette leaf. After dipping, the leaves were imaged on a stereomicroscope and the Zeiss Axio software used to determine the surface area of the leaves.

Following dipping, the chloroform was evaporated under a gentle stream of nitrogen. $100 \mu \mathrm{l}$ of bis-(Trimethylsily) trifluoroacetamide (BSTFA; Pierce) was added to each tube for derivatization, and then incubated at $80^{\circ} \mathrm{C}$ for $90 \mathrm{~min}$. All the samples were transferred to GC sample vials containing conical glass inserts and then $1 \mu \mathrm{L}$ injected on to a Varian GC-3900 gas chromatograph, equipped with a flame ionization detector (FID). The oven was programmed to maintain an initial temperature of $150^{\circ} \mathrm{C}$ for $1 \mathrm{~min}$, increased to $300^{\circ} \mathrm{C}$ at a rate of $4^{\circ} \mathrm{C} / \mathrm{min}$, and then maintained at $300^{\circ} \mathrm{C}$ for 15 $\min$.

\subsubsection{Complementation crosses}

Complementation crosses were performed to determine allelism between line and cer mutants. Plants used for crossing were 4-5 weeks old. For the female parent, all the flowers and siliques near the top portion were removed using small scissors. Flower buds, typically 2-3, were identified whose anthers had not begun to shed pollen onto the stigma. The rest of the flower buds were removed. Crossing tweezers were used to open each flower bud. All the sepals, petals and anthers were removed, leaving only the 
carpels intact. An open flower from the male parent was removed using crossing tweezers and the convex surface of the anthers was brushed against the stigmatic surface of the exposed carpels on the female parent. Crosses were covered in plastic wrap until the siliques turned yellow. All the siliques were collected separately in sandwich bags (DE LUXE, Mississauga ON), and allowed to dry at room temperature for at least 2 weeks before sowing. 


\subsection{Results}

\subsubsection{Survey of stem epicuticular wax crystal of cer mutants and line mutants}

Scanning electron microscopy (SEM) was performed to examine the epicuticular crystals of a collection of mutant plants with glossy stems. I analyzed 10 uncharacterized line mutants, which had been earlier identified by the Rowland lab (unpublished). For comparison, I also analyzed 11 cer mutants, which were previously reported, but in many cases the SEM images of wax crystals were not of high clarity (Koornneef et al., 1989; Aarts et al., 1995; Negruk et al., 1996; Fiebig et al., 2000; Chen et al., 2003; Pighin et al., 2004; Rashotte et al., 2004; Zheng et al., 2005; Rowland et al., 2006; Hooker et al., 2007) (Figure 6 and 7). A variety of alterations were observed in the wax crystal density and/or crystal shapes in the sets of mutants. The cuticle surface features were different for all of the cer and line mutants compared to wild-type, as described in more detail below when comparing groups of phenotypically related mutants.

\subsubsection{Wax load and composition of cer mutants and line mutants}

Gas chromatography was performed to determine the total wax load and wax composition of cer mutants, line mutants, and corresponding wild type ecotypes (Col-0 or Ler) (Table 2 and 3). As expected, all of the cer and line mutants had alterations in their total wax load and/or composition. The proportional changes in wax compositions of the cer mutants were consistent with that reported in the literature (Jenks et al., 1995; Rashotte et al., 2001). The alterations of wax compositions in the line mutants are described in more detail below when comparing groups of phenotypically related mutants. 
Figure 6 Scanning electron microscopy images of stem surfaces of Ler-WT and $11 \mathrm{cer}$ mutants

Epicuticular wax crystals on stems of 11 Arabidopsis cer mutants and the corresponding wild type ecotype (Ler-WT) were imaged by SEM and images taken at magnifications of $1500 \mathrm{X}, 5000 \mathrm{X}$, and 10000X, respectively. The scale bars are shown at the bottom of each panel. 


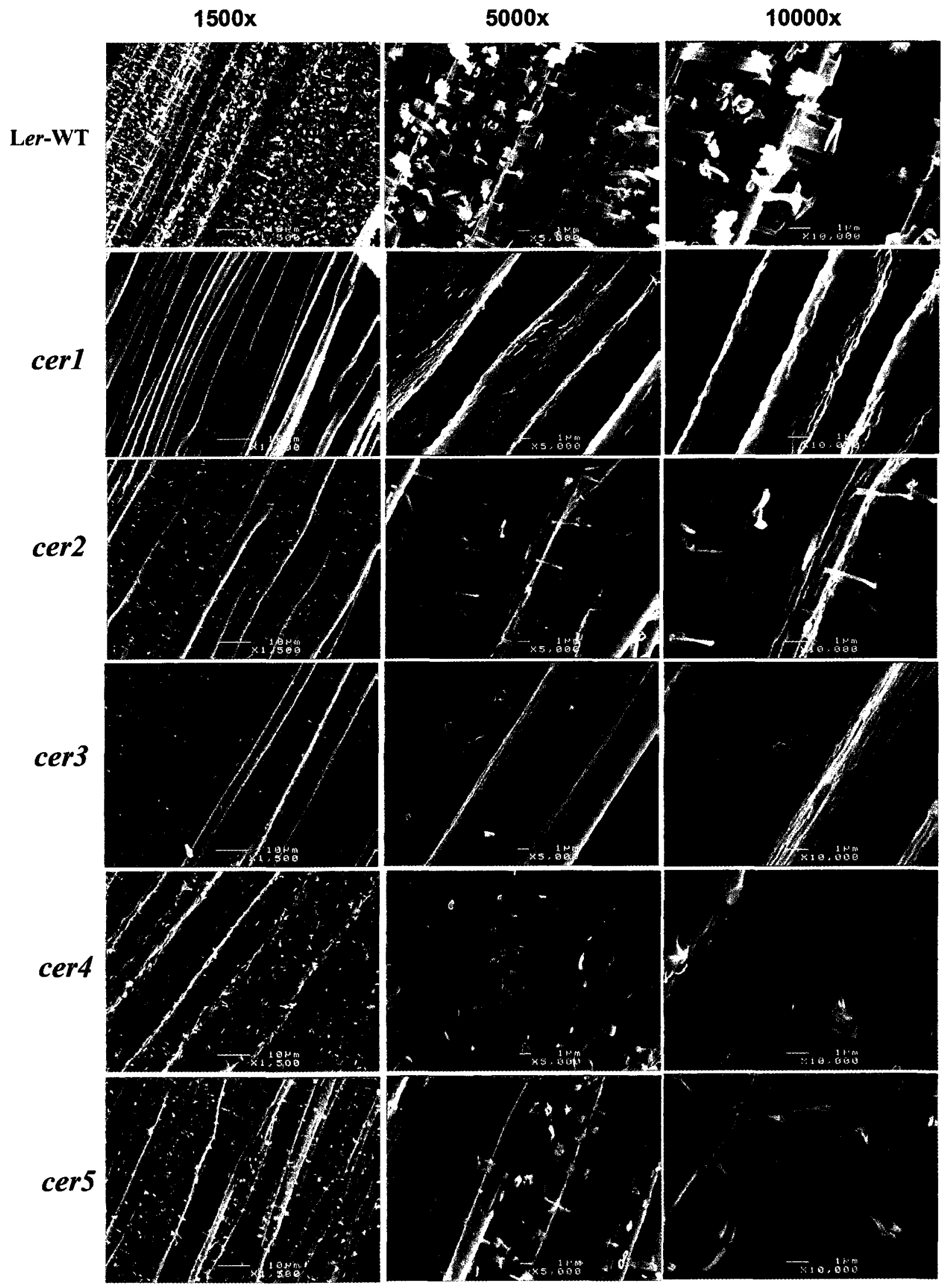




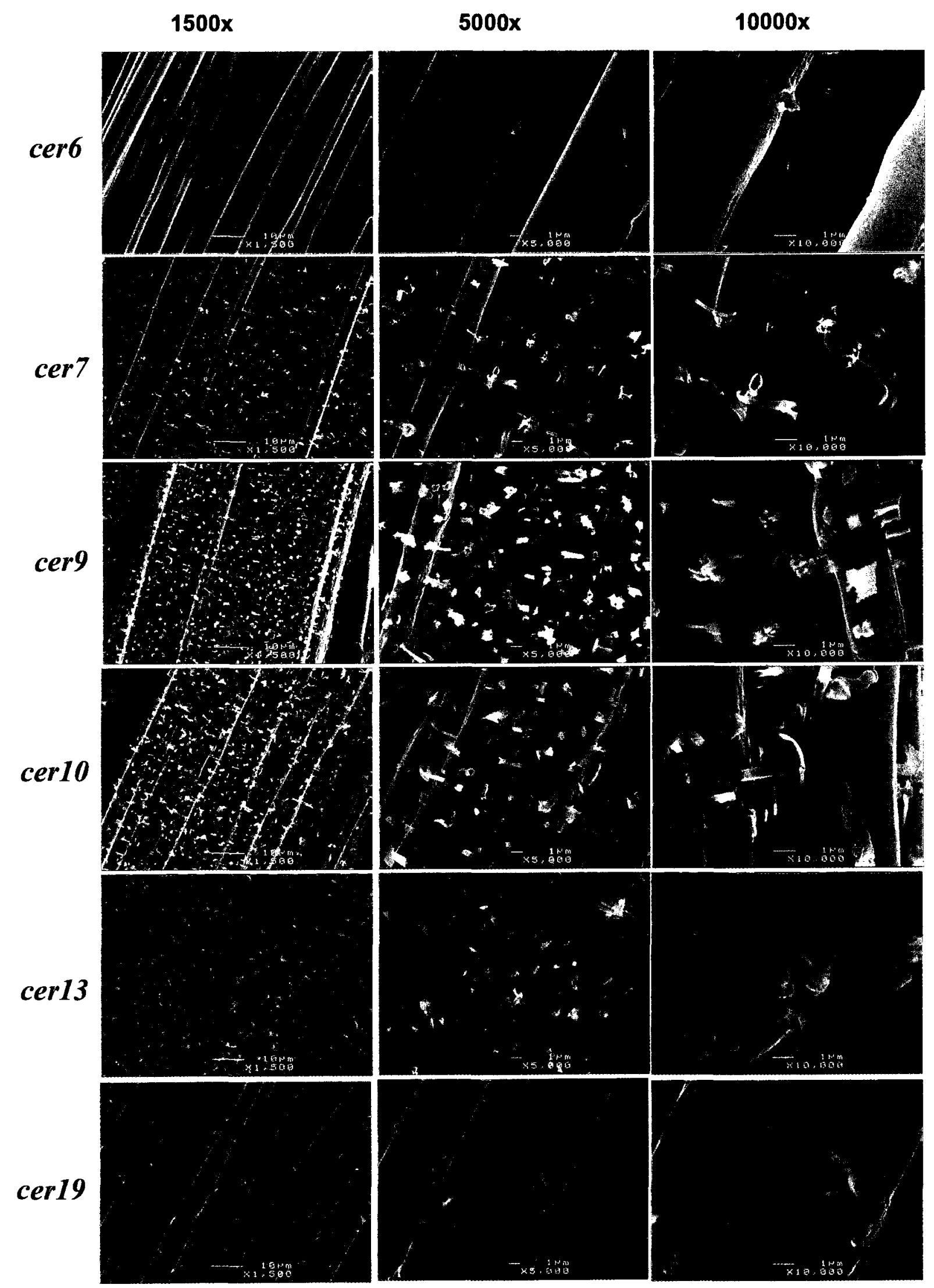


Figure 7 Scanning electron microscopy of stem surfaces of 10 line mutants and the corresponding wild-type ecotypes

Epicuticular wax crystals on stems of 10 Arabidopsis line mutants and the corresponding wild type ecotypes (Col-0 or Ler) were imaged by SEM and images taken at magnifications of $1500 \mathrm{X}, 5000 \mathrm{X}$, and $10000 \mathrm{X}$, respectively. The scale bars are shown at the bottom of each panel. 


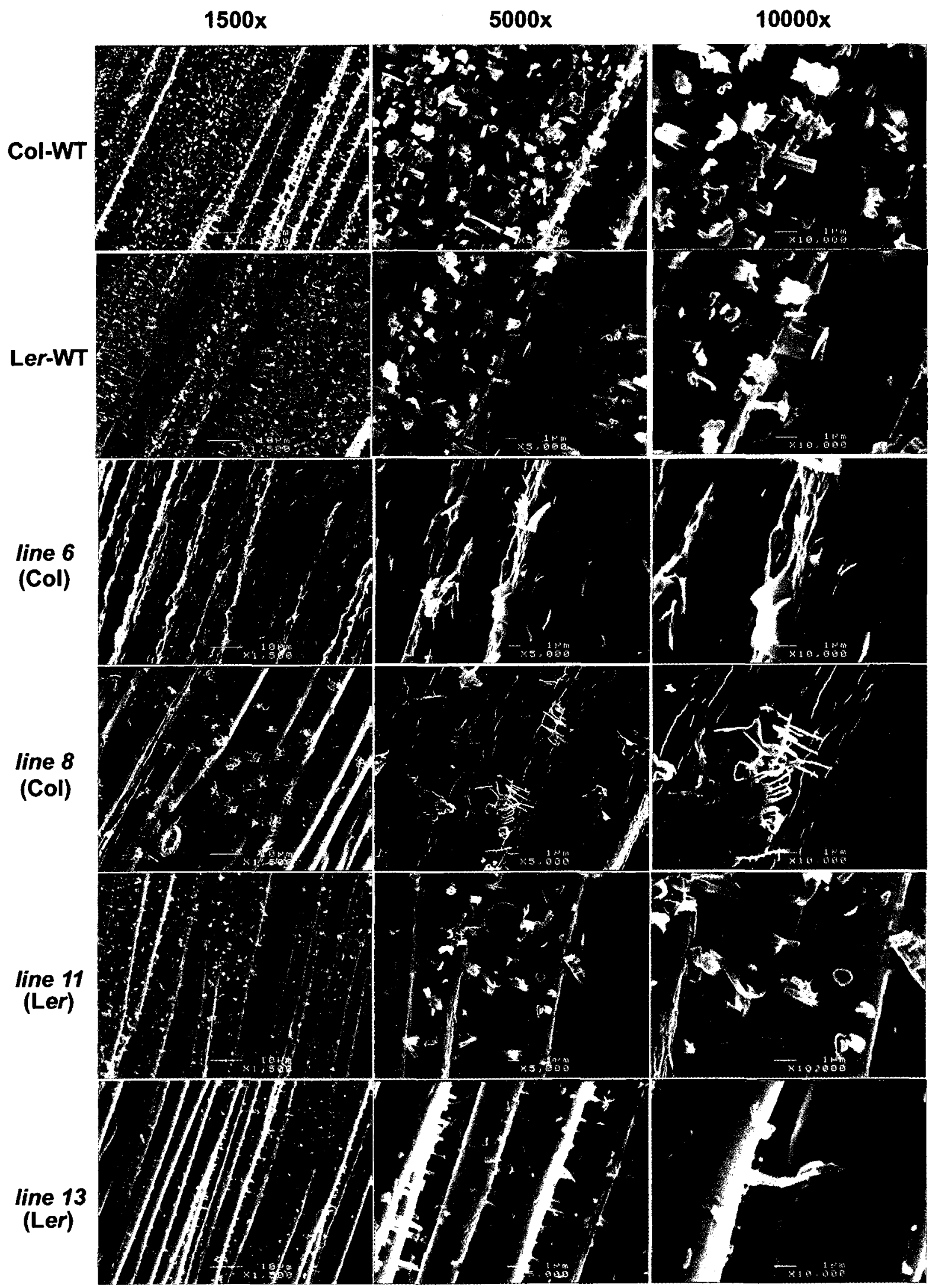




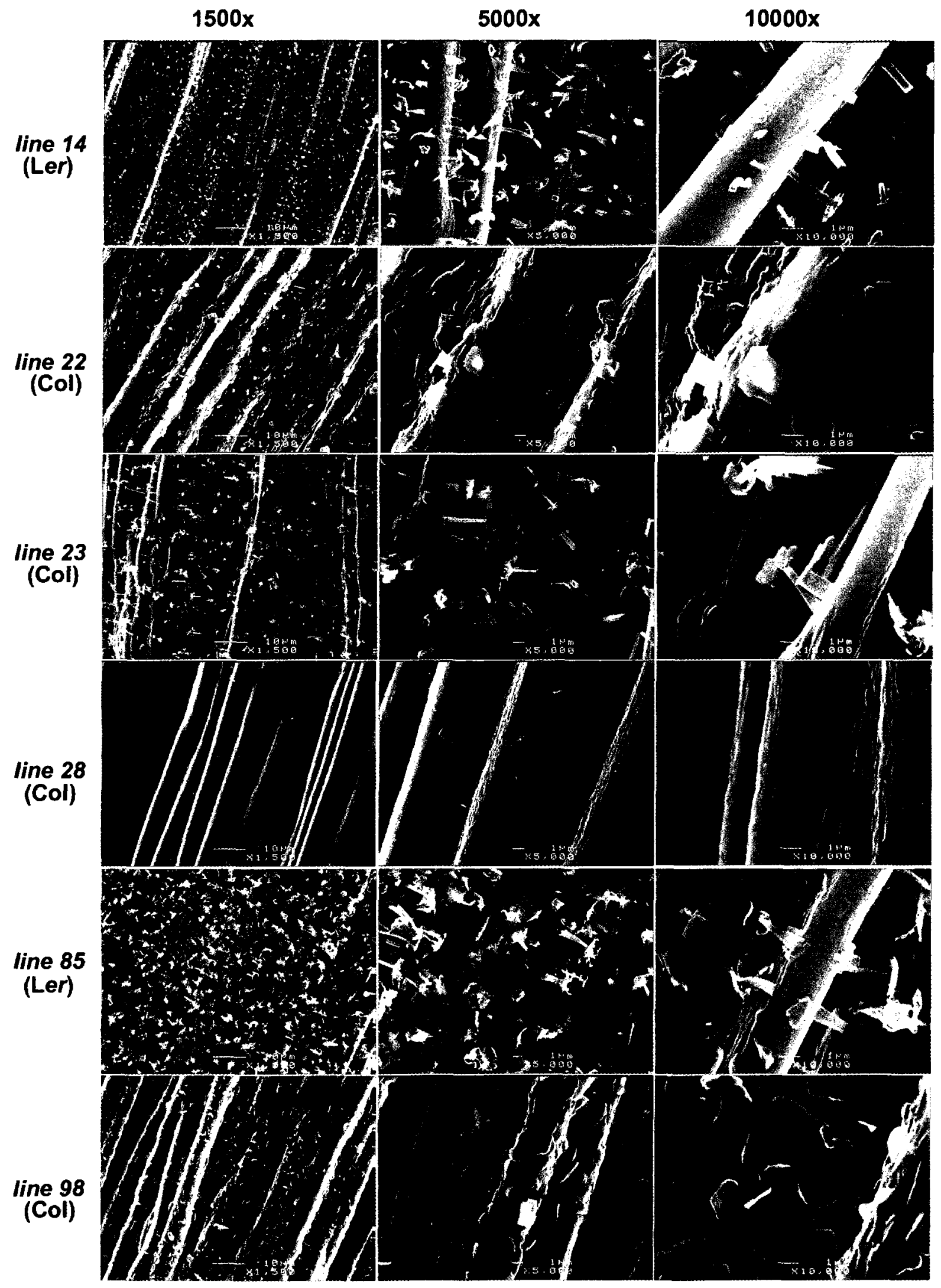


Table 2 The total wax load and wax composition from stems of cer mutants and wild type ecotypes.

\begin{tabular}{|c|c|c|c|c|c|c|c|}
\hline & $\begin{array}{l}\text { Total } \\
\text { wax } \\
\text { load }\end{array}$ & $\begin{array}{l}\text { Total } \\
\text { FFA }\end{array}$ & $\begin{array}{l}\text { Total } \\
1^{\circ} \mathrm{OH}\end{array}$ & $\begin{array}{l}\text { Total } \\
\text { ALK }\end{array}$ & $\begin{array}{l}\text { Total } \\
\text { ALD }\end{array}$ & $\begin{array}{l}\text { Total } \\
\text { KETO }\end{array}$ & $\begin{array}{l}\text { Total } \\
2^{\circ} \mathrm{OH}\end{array}$ \\
\hline Ler-WT & $\begin{array}{r}17.78 \pm \\
1.12\end{array}$ & $\begin{array}{r}0.83 \pm \\
0.04\end{array}$ & $\begin{array}{r}1.21 \pm \\
0.02\end{array}$ & $\begin{array}{r}6.87 \pm \\
0.41\end{array}$ & $\begin{array}{r}0.74 \pm \\
0.09\end{array}$ & $\begin{array}{r}1.73 \pm \\
0.11\end{array}$ & $\begin{array}{r}4.25 \pm \\
0.24\end{array}$ \\
\hline cer1 & $\begin{array}{r}4.25 \pm \\
0.98 \\
\end{array}$ & $\begin{array}{r}0.58 \pm \\
0.11 \\
\end{array}$ & $\begin{array}{r}0.78 \pm \\
0.21 \\
\end{array}$ & $\begin{array}{r}0.51 \pm \\
0.15\end{array}$ & $\begin{array}{r}0.66 \pm \\
0.02\end{array}$ & $\begin{array}{r}0.08 \pm \\
0.02 \\
\end{array}$ & $\begin{array}{r}0.35 \pm \\
0.08 \\
\end{array}$ \\
\hline cer2 & $\begin{array}{r}27.42 \pm \\
2.19 \\
\end{array}$ & $\begin{array}{r}0.78 \pm \\
0.18 \\
\end{array}$ & $\begin{array}{r}3.93 \pm \\
0.12 \\
\end{array}$ & $\begin{array}{r}11.52 \pm \\
1.46 \\
\end{array}$ & $\begin{array}{r}0.87 \pm \\
0.06 \\
\end{array}$ & $\begin{array}{r}2.11 \pm \\
0.08 \\
\end{array}$ & $\begin{array}{r}6.07 \pm \\
0.61 \\
\end{array}$ \\
\hline cer3 & $\begin{array}{r}9.36 \pm \\
1.73 \\
\end{array}$ & $\begin{array}{r}0.61 \pm \\
0.12 \\
\end{array}$ & $\begin{array}{r}1.03 \pm \\
0.17 \\
\end{array}$ & $\begin{array}{r}1.61 \pm \\
0.66 \\
\end{array}$ & $\begin{array}{r}0.08 \pm \\
0.03 \\
\end{array}$ & $\begin{array}{r}0.18 \pm \\
0.11 \\
\end{array}$ & $\begin{array}{r}0.83 \pm \\
0.42 \\
\end{array}$ \\
\hline cer4 & $\begin{array}{r}26.07 \pm \\
3.11 \\
\end{array}$ & $\begin{array}{r}0.72 \pm \\
0.02 \\
\end{array}$ & $\begin{array}{r}0.16 \pm \\
0.03 \\
\end{array}$ & $\begin{array}{r}15.34 \pm \\
1.25 \\
\end{array}$ & $\begin{array}{r}0.67 \pm \\
0.19 \\
\end{array}$ & $\begin{array}{r}2.34 \pm \\
0.21 \\
\end{array}$ & $\begin{array}{r}6.23 \pm \\
0.44 \\
\end{array}$ \\
\hline cer5 & $\begin{array}{r}12.84 \pm \\
2.59 \\
\end{array}$ & $\begin{array}{r}0.45 \pm \\
0.12 \\
\end{array}$ & $\begin{array}{r}1.17 \pm \\
0.24 \\
\end{array}$ & $\begin{array}{r}1.41 \pm \\
0.29 \\
\end{array}$ & $\begin{array}{r}0.09 \pm \\
0.01 \\
\end{array}$ & $\begin{array}{r}0.88 \pm \\
0.11 \\
\end{array}$ & $\begin{array}{r}2.11 \pm \\
0.14 \\
\end{array}$ \\
\hline cer6 & $\begin{array}{r}13.01 \pm \\
2.49 \\
\end{array}$ & $\begin{array}{r}0.58 \pm \\
0.12 \\
\end{array}$ & $\begin{array}{r}2.19 \pm \\
0.19 \\
\end{array}$ & $\begin{array}{r}1.21 \pm \\
0.27\end{array}$ & $\begin{array}{r}0.78 \pm \\
0.14 \\
\end{array}$ & $\begin{array}{r}0.11 \pm \\
0.02 \\
\end{array}$ & $\begin{array}{r}0.36 \pm \\
0.01 \\
\end{array}$ \\
\hline cer7 & $\begin{array}{r}14.12 \pm \\
3.17 \\
\end{array}$ & $\begin{array}{r}0.48 \pm \\
0.17 \\
\end{array}$ & $\begin{array}{r}1.83 \pm \\
0.19 \\
\end{array}$ & $\begin{array}{r}2.66 \pm \\
0.18 \\
\end{array}$ & $\begin{array}{r}0.14 \pm \\
0.01 \\
\end{array}$ & $\begin{array}{r}0.41 \pm \\
0.03 \\
\end{array}$ & $\begin{array}{r}1.37 \pm \\
0.15 \\
\end{array}$ \\
\hline cer9 & $\begin{array}{r}11.06 \pm \\
1.61\end{array}$ & $\begin{array}{r}1.32 \pm \\
0.28\end{array}$ & $\begin{array}{r}2.51 \pm \\
0.19 \\
\end{array}$ & $\begin{array}{r}2.01 \pm \\
0.13\end{array}$ & $\begin{array}{r}0.38 \pm \\
0.04 \\
\end{array}$ & $\begin{array}{r}0.44 \pm \\
0.02\end{array}$ & $\begin{array}{r}1.53 \pm \\
0.11 \\
\end{array}$ \\
\hline cer10 & $\begin{array}{r}14.61 \pm \\
2.05 \\
\end{array}$ & $\begin{array}{r}0.41 \pm \\
0.08 \\
\end{array}$ & $\begin{array}{r}1.01 \pm \\
0.15 \\
\end{array}$ & $\begin{array}{r}4.62 \pm \\
0.45 \\
\end{array}$ & $\begin{array}{r}0.25 \pm \\
0.2 \\
\end{array}$ & $\begin{array}{r}0.57 \pm \\
0.07 \\
\end{array}$ & $\begin{array}{r}1.63 \pm \\
0.24 \\
\end{array}$ \\
\hline cer19 & $\begin{array}{r}10.01 \pm \\
1.98\end{array}$ & $\begin{array}{r}0.82 \pm \\
0.21\end{array}$ & $\begin{array}{r}1.35 \pm \\
0.11\end{array}$ & $\begin{array}{r}1.11 \pm \\
0.04\end{array}$ & $\begin{array}{r}0.23 \pm \\
0.03\end{array}$ & $\begin{array}{r}0.27 \pm \\
0.01\end{array}$ & $\begin{array}{r}0.99 \pm \\
0.05\end{array}$ \\
\hline
\end{tabular}

FFA: Free fatty acid; $1^{\circ} \mathrm{OH}$ : Primary fatty alcohol; ALK: Alkane; ALD: Aldehyde; $2^{\circ} \mathrm{OH}$ : Secondary alcohol

All values are the means from three replicates and \pm standard deviation is shown. The amounts, presented in $\mu \mathrm{g} / \mathrm{cm}^{2}$, of the various chain lengths of the homologue series of each chemical type were combined to give the 'total' amount for each. 
Table 3 The total wax load and wax composition from stems of line mutants and wild type ecotypes.

\begin{tabular}{|l|r|r|r|r|r|r|r|}
\hline & $\begin{array}{l}\text { Total } \\
\text { wax }\end{array}$ & \multicolumn{1}{l|}{$\begin{array}{l}\text { Total } \\
\text { load }\end{array}$} & \multicolumn{1}{l|}{$\begin{array}{l}\text { Total } \\
\text { FFA }\end{array}$} & \multicolumn{1}{l|}{$\begin{array}{l}\text { Total } \\
\text { ALK }\end{array}$} & $\begin{array}{l}\text { Total } \\
\text { ALD }\end{array}$ & $\begin{array}{l}\text { Total } \\
\text { KETO }\end{array}$ & $\begin{array}{l}\text { Total } \\
2^{\circ} \mathrm{OH}\end{array}$ \\
\hline Col- WT & \multicolumn{1}{l}{$19.99 \pm$} & $0.74 \pm$ & $1.68 \pm$ & $7.75 \pm$ & $0.87 \pm$ & $1.69 \pm$ & $4.71 \pm$ \\
& 0.84 & 0.05 & 0.14 & 0.27 & 0.09 & 0.06 & 0.24 \\
\hline Ler-WT & $17.79 \pm$ & $0.83 \pm$ & $1.2 \pm$ & $6.87 \pm$ & $0.74 \pm$ & $1.73 \pm$ & $4.25 \pm$ \\
& 1.13 & 0.04 & 0.02 & 0.41 & 0.09 & 0.1 & 0.24 \\
\hline line6 & $21.54 \pm$ & $0.32 \pm$ & $0.25 \pm$ & $8.58 \pm$ & $1.4 \pm$ & $1.39 \pm$ & $3.87 \pm$ \\
& 1.15 & 0.11 & 0.11 & 1.2 & 0.84 & 0.41 & 0.56 \\
\hline line8 & $10.23 \pm$ & $0.98 \pm$ & $2.36 \pm$ & $1.18 \pm$ & $0.79 \pm$ & $0.18 \pm$ & $0.78 \pm$ \\
& 1.12 & 0.11 & 0.37 & 0.14 & 0.22 & 0.02 & 0.11 \\
\hline line11 & $11.67 \pm$ & $0.38 \pm$ & $1.57 \pm$ & $2.27 \pm$ & $0.13 \pm$ & $0.36 \pm$ & $1.13 \pm$ \\
& 3.91 & 0.2 & 0.41 & 0.53 & 0.04 & 0.1 & 0.24 \\
\hline line13 & $9.91 \pm$ & $0.57 \pm$ & $1.17 \pm$ & $2.02 \pm$ & $0.04 \pm$ & $0.24 \pm$ & $1.13 \pm$ \\
& 0.92 & 0.07 & 0.05 & 0.29 & 0.01 & 0.04 & 0.16 \\
\hline line14 & $10.25 \pm$ & $0.48 \pm$ & $1.29 \pm$ & $2.43 \pm$ & $0.16 \pm$ & $0.33 \pm$ & $1.12 \pm$ \\
& 1.49 & 0.11 & 0.48 & 0.55 & 0.1 & 0.07 & 0.18 \\
\hline line22 & $17.34 \pm$ & $0.34 \pm$ & $0.25 \pm$ & $6.14 \pm$ & $0.59 \pm$ & $1.17 \pm$ & 3.44 \\
& 3.39 & 0.12 & 0.08 & 1.59 & 0.23 & 0.29 & 0.84 \\
\hline line23 & $13.24 \pm$ & $0.49 \pm$ & $1.21 \pm$ & $2.08 \pm$ & $0.98 \pm$ & $0.27 \pm$ & $0.82 \pm$ \\
& 4.16 & 0.21 & 0.56 & 0.67 & 0.27 & 0.01 & 0.3 \\
\hline line28 & $10.16 \pm$ & $0.66 \pm$ & $1.02 \pm$ & $0.64 \pm$ & $1.1 \pm$ & $0.06 \pm$ & $0.21 \pm$ \\
& 2.21 & 0.18 & 0.21 & 0.29 & 0.12 & 0.02 & 0.04 \\
\hline line85 & $8.77 \pm$ & $0.77 \pm$ & $1.27 \pm$ & $1.07 \pm$ & $0.19 \pm$ & $0.26 \pm$ & $0.98 \pm$ \\
& 1.3 & 0.11 & 0.12 & 0.08 & 0.03 & 0.02 & 0.09 \\
\hline line98 & $18.44 \pm$ & $0.29 \pm$ & $0.20 \pm$ & $7.08 \pm$ & $1.23 \pm$ & $1.16 \pm$ & $3.27 \pm$ \\
& 1.63 & 0.16 & 0.11 & 1.01 & 0.26 & 0.15 & 0.71 \\
\hline
\end{tabular}

FFA: Free fatty acid; $1^{\circ} \mathrm{OH}$ : Primary fatty alcohol; ALK: Alkane; ALD: Aldehyde; $2^{\circ} \mathrm{OH}$ : Secondary alcohol

All values are the means from three replicates and \pm standard deviation is shown. The amounts, presented in $\mu \mathrm{g} / \mathrm{cm}^{2}$, of the various chain lengths of the homologue series of each chemical type were combined to give the 'total' amount for each. 
2.3.3 Comparisons of phenotypically similar cer and line mutants

After comparing the epicuticular wax crystal density and shape between cer and line mutants based on SEM images and the cuticular wax compositions of cer and line mutants based on GC data, I predicted the following: 1) line6, line22, and line98 are allelic with cer4 (Figure 8), 2) line11, line13, and line14 are allelic with cer3 (Figure 9), 3) line28 is allelic with cer1 (Figure 10), 4) line23 is allelic with cer7 (Figure 11), and 5) line8 and line85 are novel loci affecting cuticular wax production (chapter 3 ).

SEM images of line6, line22, and line98 have a similar wax crystal alteration as found for cer4. They all contain platelet-shape wax crystals, while the typical tube-shape wax crystals on wild-type were missing (Figure 8A). The total stem wax loads of all four mutants are similar to corresponding wild-type ecotype (Table 2, 3). Primary alcohols with chain lengths $\mathrm{C} 26-\mathrm{C} 30$ of line6, line22, line98, and cer4 were dramatically reduced, while all the individual components of the alkane forming pathway were slightly increased (Figure 8B). There was considerable variability of the $\mathrm{C} 30$ aldehyde, but this peak may be co-eluting with other unknown compounds. The F1 generations of complementation crosses between each line mutant and cer mutant had a glossy phenotype, confirming that line6, line22, and line98 are allelic to cer4 (Table 4).

The wax crystal density of line11, line13, line14, and cer3 were significantly reduced and were characterized by dispersed longitudinal rodlets with a cap on top (Figure 9A). The total stem wax loads of line11, line13, and line14 were highly reduced. All the components in alkane forming pathway are reduced, while primary alcohols are increased, especially the C30 primary alcohol (Figure 9B). These wax composition defects are similar to that of cer3. A complementation cross test confirmed that line13 is 
allelic to cer3 (Table 4). Because these mutants are partially sterile, the complementation crosses between line11, line14, and cer3 were not successful and no definitive conclusion on allelism could be made.

The wax crystals of the stem surfaces of line 28 and cer1 are completely absent (Figure 10A). The total stem wax load and all wax components of both these two mutants are highly reduced, especially the major wax components, such as C29 alkane, C29 secondary alcohol, and C29 ketone (Table 2, 3; Figure 10B). Complementation crosses confirmed that line28 is allelic to cer1 (Table 4).

The SEM images are very similar between line23 and cer7, characterized by similar reductions in wax crystal density and slender longitudinal rodlets capped with dendritic formations (Figure 11A). line23 and cer7 have similar reductions in C29 alkanes, secondary alcohols, and dramatic increases in C24 primary alcohols and C33 alkanes (Figure 11B). However, there were some differences. For example, cer7 had a major increase of C30 primary alcohols while line23 is decreased in C30 primary alcohols relative to wild-type. Also cer7 had a reduction in C30 aldehydes, while line23 was not significantly affected in this chemical class relative to wild-type. The F1 plants derived from complementation crosses between line23 and cer7 had glaucous (waxy) stems, indicating that line23 is in fact not allelic to cer7 (Table 4). 
Figure 8 Comparison of wax crystal shape and cuticular wax composition between line6, line22, line98 and cer4

(A) Scanning electron microscopy images of line6, line22, line98, cer4 and the corresponding wild type ecotypes. The SEM images were taken at magnifications of 1500X, 5000X, and 10000X, respectively. The scale bars are shown at the bottom of each panel.

(B) Stem cuticular wax composition of line6, line22, line98, cer4 and corresponding wild type ecotypes sorted by chemical type.

All values are the means, presented in $\mu \mathrm{g} / \mathrm{cm}^{2}$, derived from three replicates $(n=3)$. Error bars represent the standard deviation. 


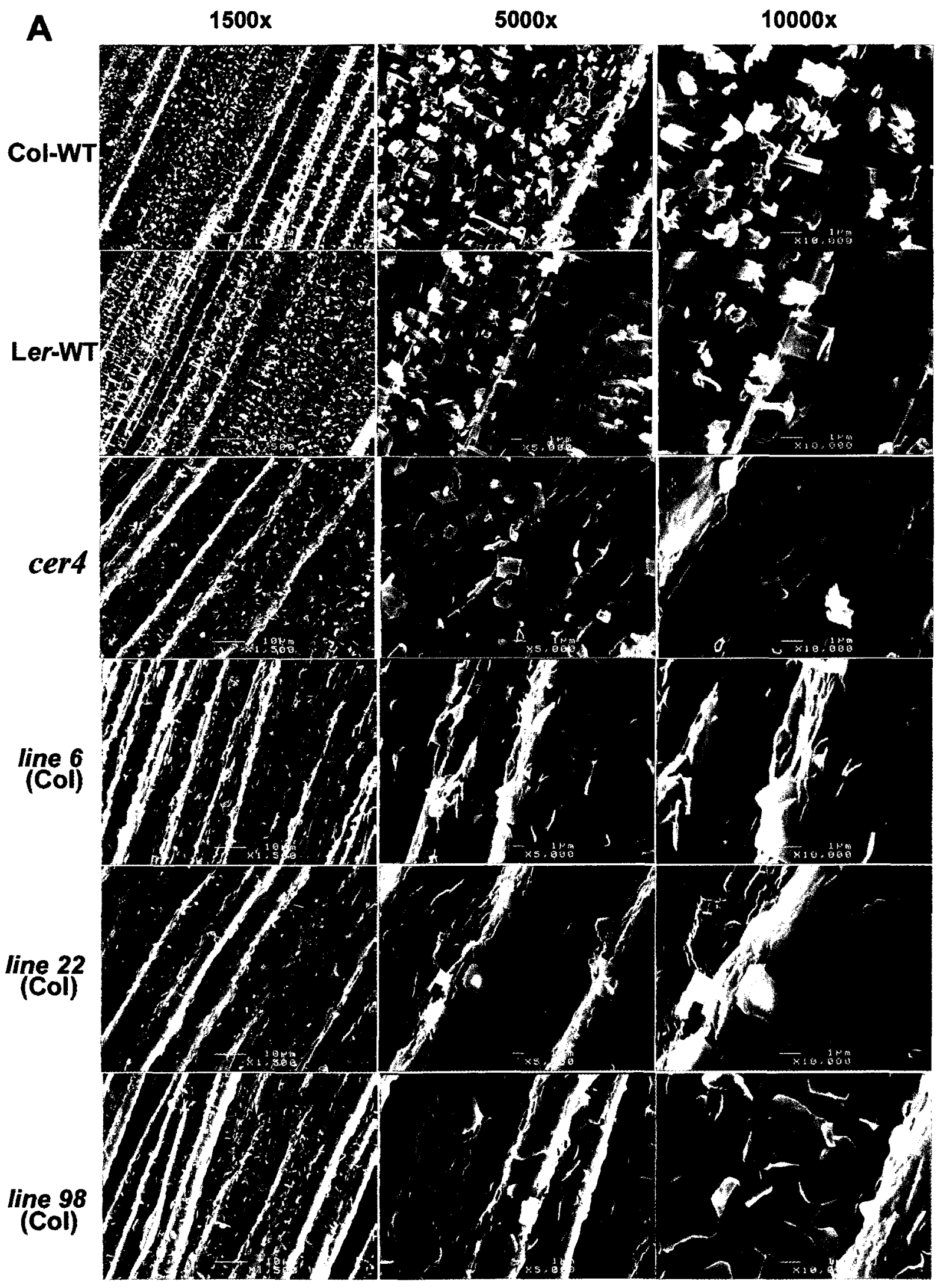




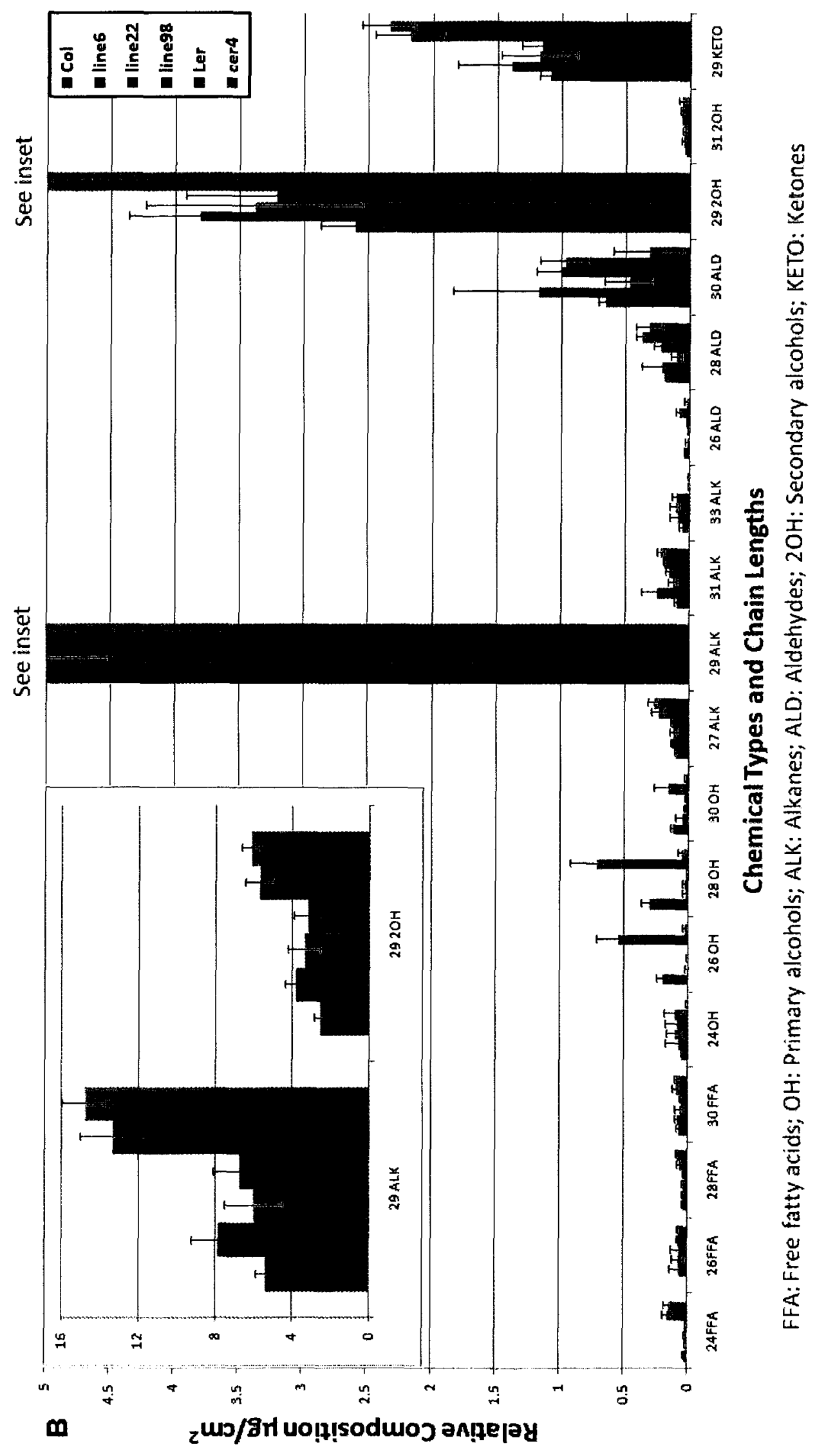


Figure 9 Comparison of wax crystal shape and cuticular wax composition between line11, line13, line14, and cer3.

(A) Scanning electron microscopy images of line11, line13, line14, cer3 and the corresponding wild type ecotypes. The SEM images were taken at magnifications of 1500X, 5000X, and 10000X, respectively. The scale bars are shown at the bottom of each panel.

(B) Stem cuticular wax composition of line11, line13, line14, cer3 and corresponding wild type ecotypes sorted by chemical type.

All values are the means, presented in $\mu \mathrm{g} / \mathrm{cm}^{2}$, derived from three replicates $(n=3)$. Error bars represent the standard deviation. 


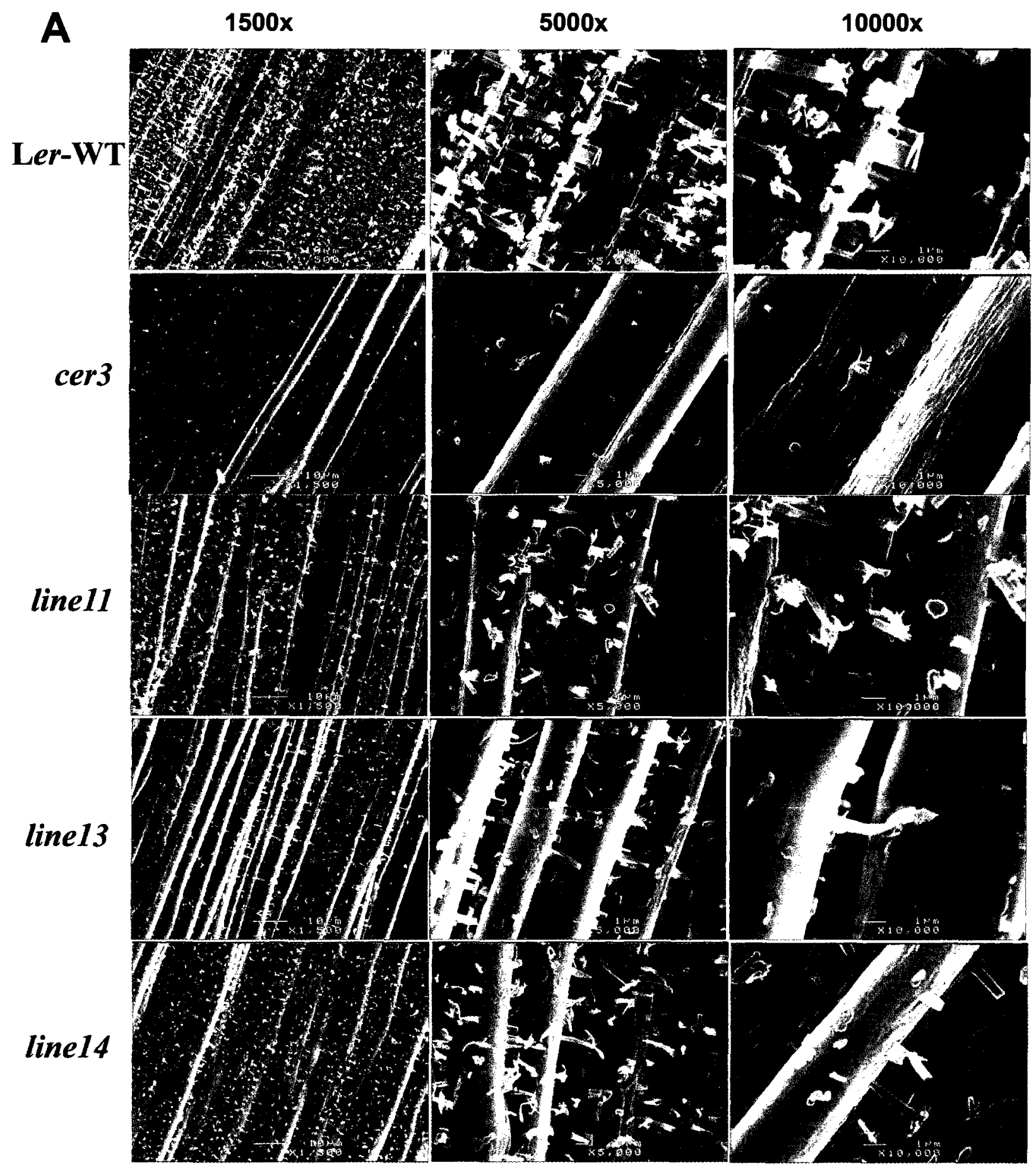




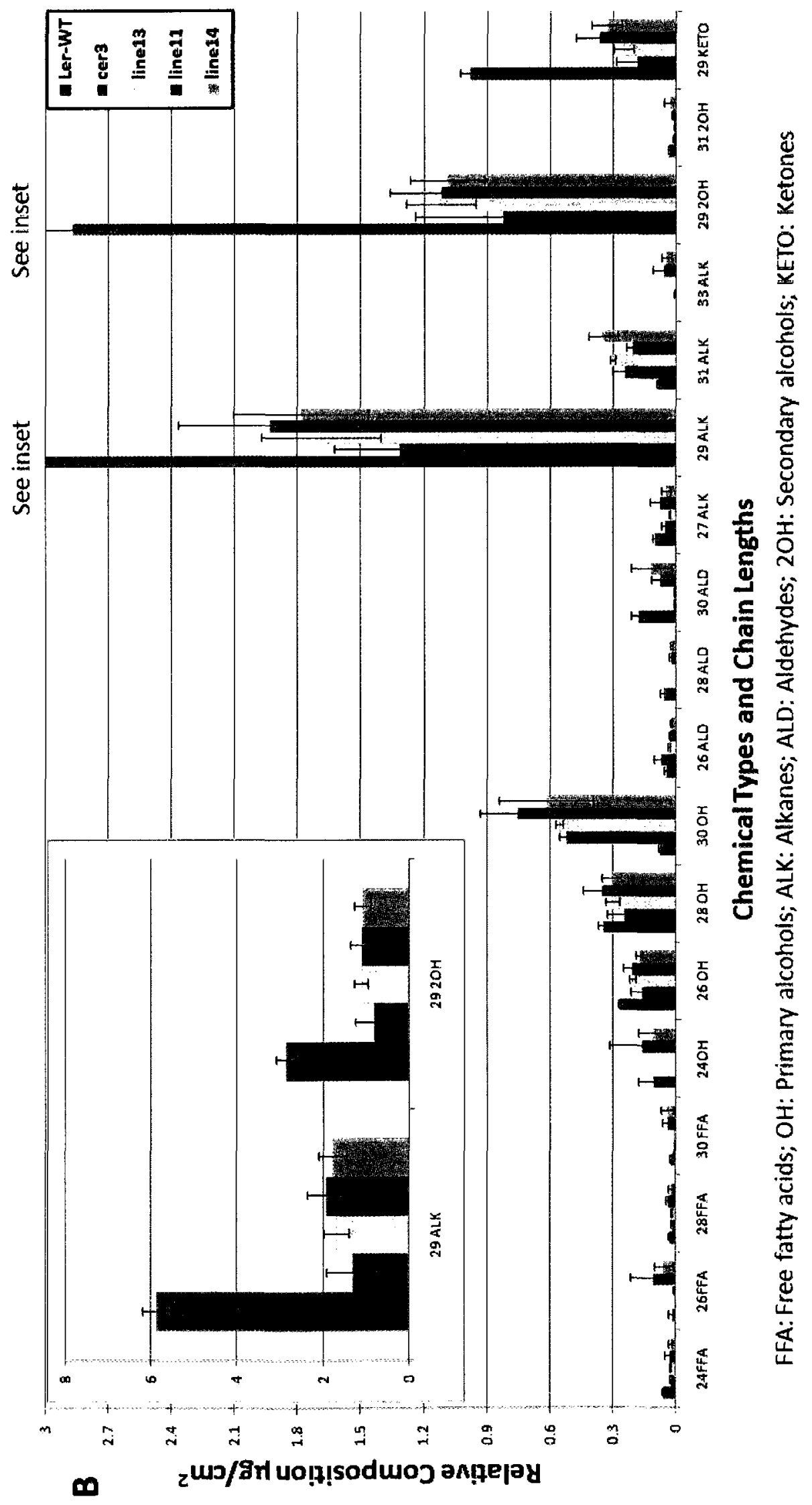


Figure 10 Comparison of wax crystal shape and cuticular wax composition between line28 and cer1.

(A) Scanning electron microscopy images of line28, cer1 and the corresponding wild type ecotype. The SEM images taken at magnifications of $1500 \mathrm{X}, 5000 \mathrm{X}$, and $10000 \mathrm{X}$, respectively. The scale bars are shown at the bottom of each panel.

(B) Stem cuticular wax composition of line28, cer1 and corresponding wild type ecotype sorted by chemical type.

All values are the means, presented in $\mu \mathrm{g} / \mathrm{cm}^{2}$, derived from three replicates $(n=3)$. Error bars represent the standard deviation. 


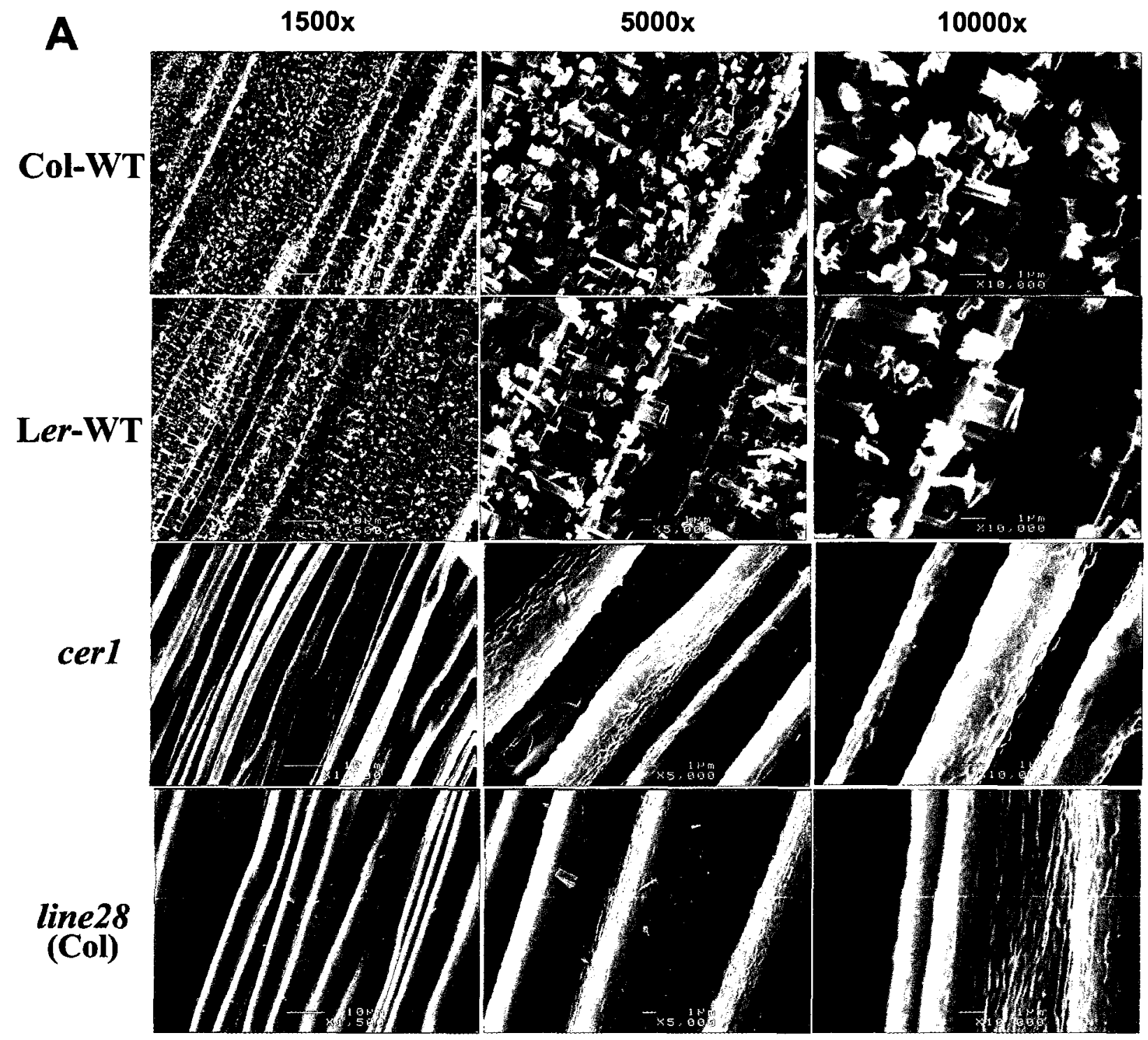




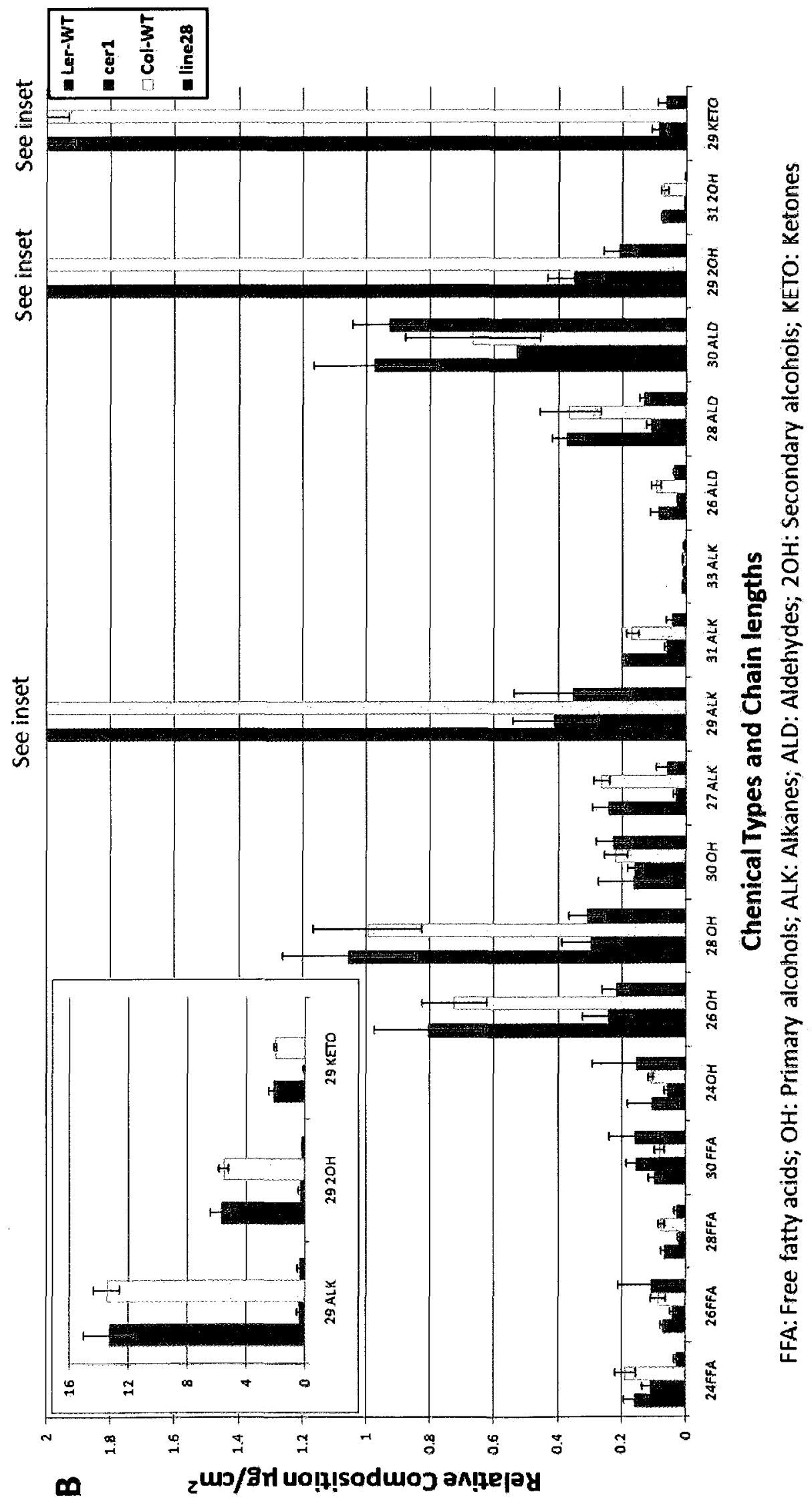


Figure 11 Comparison of wax crystal shape and cuticular wax composition between line23 and cer7.

(A) Scanning electron microscopy images of line23, cer7 and the corresponding wild type ecotypes. The SEM images taken at magnification of 1500X, 5000X, and 10000X, respectively. The scale bars are shown at the bottom of each panel.

(B) Stem cuticular wax composition of line23, cer7 and corresponding wild type ecotypes sorted by chemical type.

All values are the means, presented in $\mu \mathrm{g} / \mathrm{cm}^{2}$, derived from three replicates $(n=3)$. Error bars represent the standard deviation. 


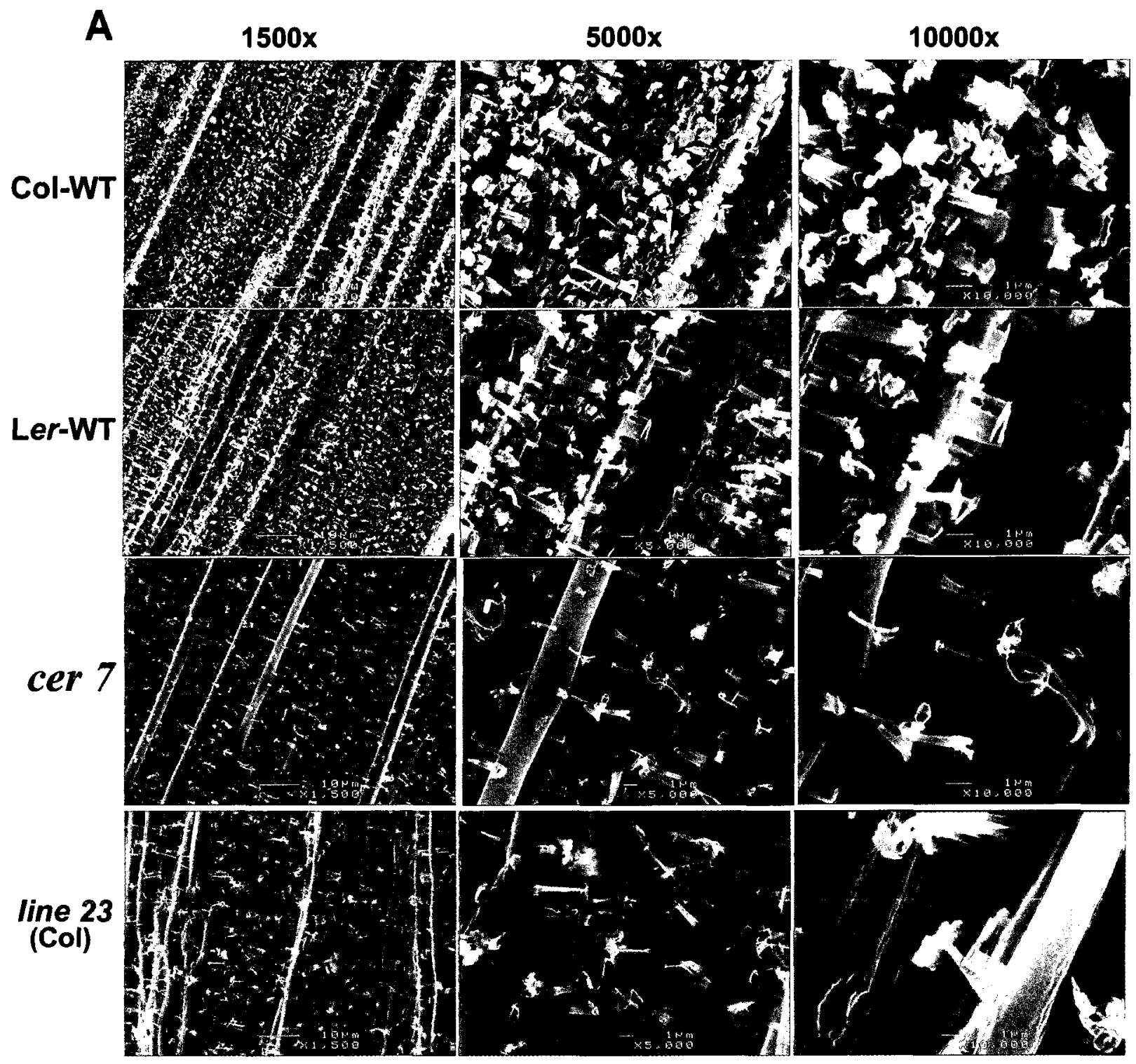




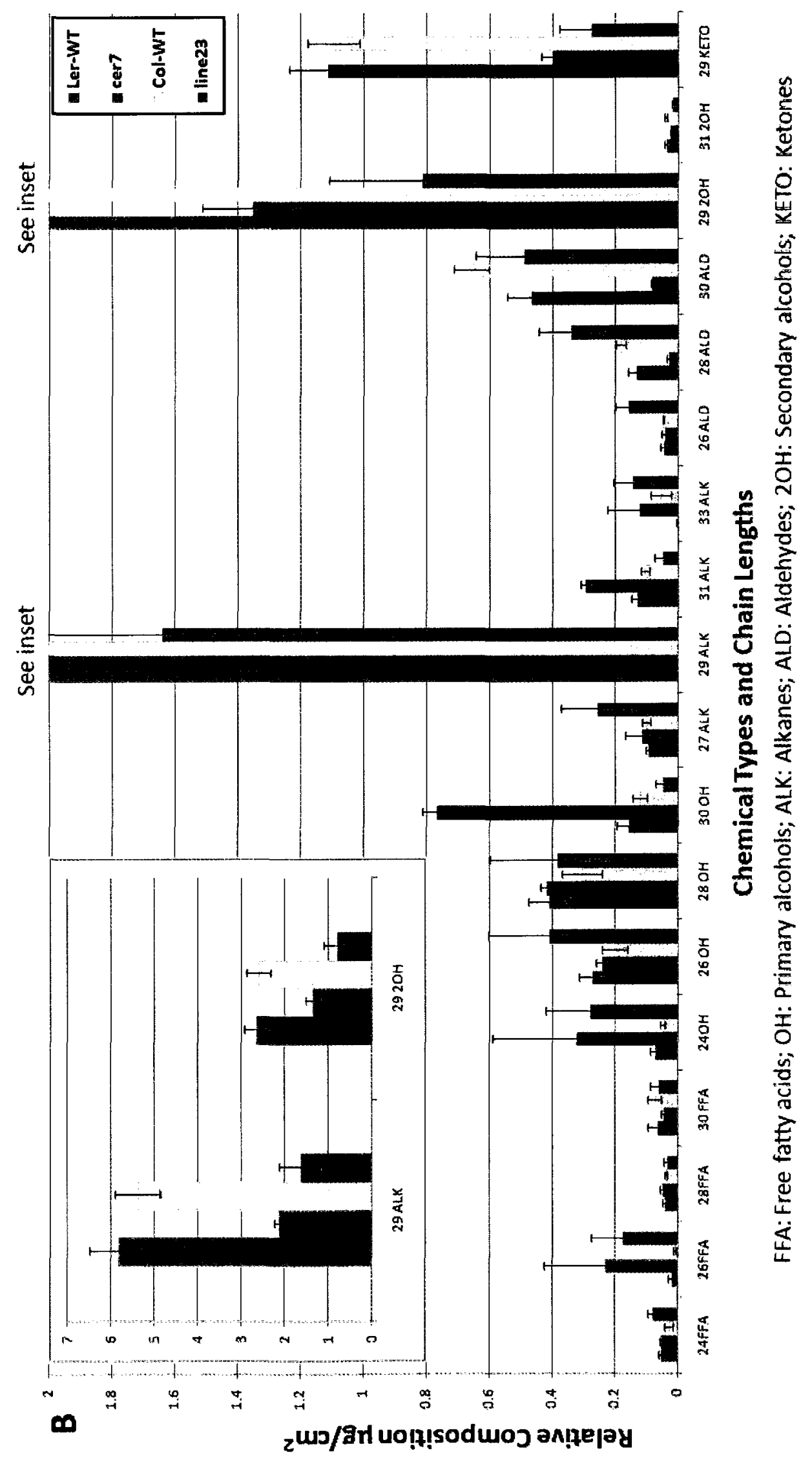


Table 4 Complementation crosses between similar line mutants and cer mutants

\begin{tabular}{|c|c|c|c|}
\hline$q \times 0$ & $\begin{array}{l}\text { No. of successful } \\
\text { crosses/total crosses }\end{array}$ & $\begin{array}{l}\text { F1 generation } \\
\text { phenotype }\end{array}$ & Allelism \\
\hline line $6 \mathrm{X}$ cer 4 & $2 / 3$ & Glossy & Yes \\
\hline cer $4 \times$ line 6 & $1 / 3$ & Glossy & Yes \\
\hline line $22 \times$ cer 4 & $1 / 4$ & Glossy & Yes \\
\hline cer4 $\mathrm{X}$ line22 & $1 / 3$ & Glossy & Yes \\
\hline line $98 \times$ cer 4 & $3 / 3$ & Glossy & Yes \\
\hline cer4 $X$ line98 & $2 / 3$ & Glossy & Yes \\
\hline line $13 \times$ cer 3 & $6 / 12$ & Glossy & Yes \\
\hline cer3 $\times$ line 13 & $4 / 9$ & Glossy & Yes \\
\hline line $28 \times$ cer1 & $1 / 11$ & Glossy & Yes \\
\hline line $23 \times$ cer7 & $2 / 2$ & Waxy & No \\
\hline cer7 $X$ line23 & $1 / 2$ & Waxy & No \\
\hline
\end{tabular}




\subsection{Discussion}

The cuticular wax phenotypes of a set of new wax deficient mutants, designated line mutants were compared to previously characterized Arabidopsis cer mutants. Sets of phenotypically similar line and cer mutants were crossed to test for allelism. From this analysis, I made the following conclusions: 1) line6, line22, line98, and cer4 are allelic (Figure 8A, B; Table 4), 2) line13 and cer3 are allelic (Figure 9A, B; Table 4), 3) line28 and cer1 are allelic (Figure 10A, B; Table 4), 4) line23 and cer7 are not allelic (Figure 11A, B; Table 4). Line 8 and line 85 were predicted to be novel alleles and are the subject of Chapter 3.

CER4 encodes an alcohol-forming FAR, which is the major FAR responsible for primary alcohol formation in Arabidopsis stems (Hannoufa et al., 1993; Jenks et al., 1995; Rowland et al., 2006). To date, several cer4 mutants lines in different wild-type background have been identified and characterized (Ler-cer4-1, WS-cer4-2, Col-cer4-3, Col-cer4-4) (Rowland et al., 2006). However, these are all T-DNA insertion mutants except for cer4-1, which is a deletion of most of the gene. Since line6, line22, and line98 are all EMS mutagenized mutants, sequencing the CER4 gene in these mutants may reveal important amino acids for enzyme activity or stability. Characterization of independently derived mutant alleles of HOTHEAD revealed important amino acids for activity of the encoded FAD-containing oxidoreductase involved in cutin formation (Krolikowski et al., 2003). A similar approach could be taken with CER4 as part of a structure function analysis.

CER3, which is also called WAX2NRE/FLP1, has been cloned, but its function remains enigmatic (Chen et al., 2003; Rowland et al., 2007). Also, the predicted protein 
is related to the CER1 gene product, whose function is also currently unknown (Aarts et al., 1995). The sequences of the two predicted proteins contain His-rich motifs characteristic of a class of integral membrane enzymes, and the $\mathrm{N}$-terminal of CER3, which has six putative transmembrane domains, is highly related to that of CER1 (Chen et al., 2003; Rowland et al., 2007). Both CER3 and CER1 protein sequences suggest enzymatic roles in wax biosynthesis and the phenotypes of the mutants suggest roles in the upstream part of the alkane-forming pathway. This is the least understood part of cuticular wax biosynthesis. The identification of line13 and line28 as mutant alleles of CER3 and CER1, respectively, may be useful for further molecular and biochemical characterization of CER3 and CER1. For example, the mutations may reside in the Hisrich motifs of the predicted proteins.

It was found that line23 and cer7 are not allelic. The wax composition profile of line23 does not precisely match any of the cer mutants (this study; Jenks et al., 1995; Rashotte et al., 2001), but there was considerable variability in our wax composition analysis of line23 and this could be masking phenotypic relationships. Products of both the alcohol-forming and alkane-forming pathways appear to be affected and thus the LINE23 protein may have an upstream role in the biosynthetic pathways, or affect transport or secretion of waxes. It is possible that line23 is a novel cer loci, but more thorough analysis of line23 wax composition and tests of allelism are required.

In summary, the SEM and GC methods were effective in phenotypically characterizing a collection of new wax-deficient mutants, such that they could be grouped together for subsequent tests of allelism by genetic complementation crosses. The Rowland lab has a much larger collection of uncharacterized mutants that could be 
characterized in this manner. SEM analysis of plant surfaces is a relatively quick and easy way to phenotypically group mutants. However, the wax composition analysis is quite time-consuming and subject to distortion when low numbers of repeats are analyzed. A large-scale crossing strategy may be a more prudent approach, after general phenotypic groupings are assigned following imaging of wax crystals by SEM. 


\section{Chapter 3 Characterization and Mapping of Two Novel eceriferum Mutants}

\subsection{Introduction}

\subsubsection{Map-based cloning}

Map-based cloning, also known as positional cloning, is widely used to identify the underlying genetic cause of a mutant phenotype by identifying the mutated gene responsible for it (Jander et al., 2002). Map based cloning is based on the fact that genetic recombination and physical distance are directly related. As recombination events between homologous chromosomes decrease, so does the physical distance (Bortiri et al., 2006).

In the past several years, map-based cloning has become a relatively straightforward and time efficient process in Arabidopsis thaliana because of three breakthroughs. First, the entire Columbia (Col-0) Arabidopsis genome has been sequenced (The Arabidopsis Genome Initiative, 2000). Secondly, the partial sequencing of Landsberg erecta (Ler) and other ecotypes which revealed a wide selection of randomly distributed genetic markers polymorphisms available for analyses (Alonso and Ecker, 2006). Third, the methods for rapidly detecting DNA polymorphisms have improved (Jander et al., 2002).

Col-0 crossed to Ler (Col- $0 \times$ Ler) is the most commonly used combination for mapping purposes. Thousands of DNA polymorphisms between these two ecotypes have been identified, which allows easy design of new molecular markers (Lukowitz et al., 2000). Simple sequence length polymorphisms (SSLPS) are the most useful markers for first-pass mapping. The SSLP markers exploit the variability of short repetitive sequences in genomes. Additionally, cleaved amplified polymorphic 
sequences (CAPs) and derived CAPs (dCAPs) are also effective markers. CAPs markers exploit polymorphic restriction endonuclease sites, while dCAPs marker can exploit almost all single nucleotide changes for mapping purposes. All of these markers are codominant and can be visualized quickly and inexpensively using polymerase chain reaction (PCR), followed by DNA digestion with restriction endonucleases in the case of CAPs and dCAPs markers, and then gel electrophoresis (Peters et al., 2003).

By taking advantage of a sequenced Arabidopsis genome and a large collection of genetic markers, map-based cloning becomes a relatively straightforward and timeefficient process. The mapping process can be generally divided into four steps. The first step in map-based cloning is to generate a mapping population. The mutant (e.g. in Col-0 ecotype) is out-crossed to another ecotype (e.g. in Ler ecotype), and then F1 individuals are allowed to self-pollinate. F2 seeds are collected for growth of the mapping population and plants identified that are homozygous mutant (in the case of recessive mutations). Linkage tests to markers polymorphic spread over the five Arabidopsis chromosomes are determined during this stage and a three-point cross used to define flanking molecular markers. A dense collection and variety of genetic markers and a larger F2 population are used for fine-resolution mapping in order to narrow down the location of the mutated gene. Once a small genomic region containing the mutation of interest has been determined, typically three procedures are undertaken to identify the gene. One method is sequencing of candidate genes, a second is molecular complementation by transforming wile-type candidate genes into the mutant line(s), and the third is to perform a genetic complementation cross of the mutant with plants that have the candidate genes knocked out by a T-DNA insertion. 


\subsubsection{Physiological experiments}

The cuticle is a lipophylic barrier that protects plants from damage caused by abiotic and biotic stresses. Loss-of-function mutants that affect cuticular wax biosynthesis typically have glossy stems due to altered light reflection from the epicuticular wax crystals. Some of these mutants also display additional phenotypes, such as stunted growth, elevated transpiration rate, increased sensitivity to chemicals, morphological irregularities in cell shape, and strongly perturbed cuticle permeability (Jenks et al., 2002; Yephremov and Schreiber, 2005; Nawrath, 2006). The movement of water between the outer cell wall of the epidermis and the atmosphere adjacent to the plant is largely controlled by the plant cuticle and stomata. Transportation across the cuticle is controlled through simple diffusion along a concentration gradient (Riederer and Schreiber, 2001).

In order to investigate the integrity and permeability properties of the cuticle, specific physiological assays have been developed. These include the fresh water loss assay, chlorophyll leaching assay, and toluidine blue (TB) assay (Lolle et al., 1997; Wellesen et al., 2001; Tanaka et al., 2003). Studies indicate that the epicuticular wax and inner cuticular layers highly influence the rate of water loss (Aharoni et al., 2004). Therefore, water loss can be taken as a measure of cuticular permeability. The mechanism of chlorophyll leaching assay is similar to the water loss assay, except that the non-polar molecule chlorophyll is used. Toluidine blue (TB) is a hydrophilic dye that is generally used for histological staining of plant cell walls. Leaf surfaces without a functional cuticle are more permeable to TB and stain blue (Tanaka et al, 2003). 
Therefore applying the TB assay can reveal cuticle defective mutants even as a forward genetic screen.

Line8 and line85 were described in Chapter 2 and speculated to be novel wax deficient loci based on their surface wax crystals and wax composition. The objective of this chapter was to further characterize line8 and line85. The specific chromosome locations of line 8 and line 85 were determined by linkage analysis to molecular markers. Allelism test with nearby cer loci revealed that line 8 and line 85 were unique alleles of cer6 and cer19, rather than novel loci. The permeability defects of these mutants were measured to gain further insights into the nature of the cuticular defects. 
3.2 Materials and Methods

3.2.1 Plant materials and growth conditions

Plants were grown in a semi-controlled growth room (Nesbitt Biology Building, Room $113 \mathrm{~A}$ ) with a continuous light photoperiod at approximately $22^{\circ} \mathrm{C}$, which was controlled by a Mitsubishi air conditioner.

\subsubsection{Plant genomic DNA extraction}

Plant genomic DNA was extracted in two different ways. All the DNA samples used for first-pass mapping were extracted using the 'Whatman FTA protocol'. A young leaf was directly pressed onto a Whatman FTA classic card (Cat. No. WB120205; Whatman International Ltd, England). A piece of parafilm was laid over the leaf. The end of a $50 \mathrm{~mL}$ falcon tube was used to mash the leaf sample. The material on the FTA card was allowed to dry overnight at room temperature. A 1.2mm Micro-Punch (Harris) was used to punch each sample disc into a tube for subsequent PCR amplification of DNA markers. $50 \mu \mathrm{L}$ of FTA purification reagent $(10 \mathrm{mM}$ Tris- $\mathrm{HCl}, \mathrm{PH} 7.5,2 \mathrm{mM}$ EDTA, PH $8.0,0.1 \mathrm{~mL} / 100 \mathrm{~mL}$ Tween 20 ) was added to each tube containing the sample disk and incubated for 5 minutes at room temperature. The solution was then removed and this step was repeated. $200 \mu \mathrm{L}$ of $1 \mathrm{X}$ TE buffer ( $10 \mathrm{mM}$ Tris-HCl, $0.1 \mathrm{mM}$ EDTA, PH 8.0$)$ was added to each tube and incubated for 5 minutes at room temperature. This solution was removed and this step was repeated. All the reaction tubes containing the prepared disks were dried overnight at room temperature for PCR amplification.

The genomic DNA of samples used for fine-resolution mapping and sequencing was extracted by the 'Quick and Dirty protocol'. One rosette leaf was removed and placed into a $1.5 \mathrm{~mL}$ microcentrifuge tube. A pestle was used to grind samples by hand 
for about 15 seconds. $400 \mu \mathrm{L}$ of extraction buffer $(200 \mathrm{mM}$ Tris- $\mathrm{HCl}, \mathrm{pH} 7.5,250 \mathrm{mM}$ $\mathrm{NaCl}, 25 \mathrm{mM}$ EDTA, $0.5 \%$ SDS) was added and vortexed for 5 seconds. After all the samples were ground, the mixtures were centrifuged at $15000 \mathrm{rpm}$ in a microcentrifuge for 5 minutes. The supernatant was transferred to a new tube and $400 \mu \mathrm{L}$ of isopropanol was added. The solution was vortexed for 2 seconds and incubated for $20-30 \mathrm{~min}$ at room temperature. The solution was centrifuged at $15000 \mathrm{rpm}$ for $5 \mathrm{~min}$ and the supernatant was discarded. $500 \mu \mathrm{L}$ of $70 \%$ ethanol was added to each tube to remove excess salts and the pellet was allowed to dry overnight. The pellet was dissolved in $\mathbf{5 0}$ $\mu \mathrm{L}$ of deionized water (Sigma).

\subsubsection{Strategy for designing primers for amplification of polymorphic markers}

First, all the files for the Arabidopsis polymorphism collection and the Ler sequence were downloaded from the TAIR website: http://www.arabidopsis.org. Second, polymorphisms were chosen based on position and Insertion/Deletion (InDel) size. Usually, InDels were chosen that a difference in size of 10-40bp between Col-0 and Ler. Third, the immediate flanking sequences were used to search the Col-0 sequence using the TAIR website BLAST program (http://www.arabidopsis.org/Blast/index.jsp), choosing $A$. thaliana GB genomic (DNA) database. The 'hit' from the result was checked for consistency with the information from the original downloaded files, such as BAC name and left-coordinate number. The sequence surrounding the polymorphism was obtained from TAIR Sequence Viewer (http://www.arabidopsis.org/servlets/sv). The total sequence copied was around $200 \mathrm{bp}$. This region was pasted into the Primer 3 website: http://frodo.wi.mit.edu/ to design primers (parameters minimum size: 20; optimum size: 25 ; maximum size: 27 ). It was important to verify the polymorphism was 
present in the amplified region from Col-0 and Ler genomic sequences. For this, a pairwise BLAST using the TAIR BLAST program was used to compare the Col-0 amplified product sequence and the Ler amplified product sequence, which was downloaded as described above.

\subsubsection{PCR conditions}

An Eppendorf AG 22331 Hamburg thermo cycler (No. 5341, Germany) was used to amplify DNA templates. Each $20 \mu \mathrm{L}$ volume PCR reaction mixture consisted of $2 \mu \mathrm{L}$ of $5 \mathrm{ng} / \mu \mathrm{L}$ DNA template, $2 \mu \mathrm{L}$ of $10 \mathrm{mM}$ reverse primer, $2 \mu \mathrm{L}$ of $10 \mathrm{mM}$ forward primer, $2 \mu \mathrm{L}$ of $10 \mathrm{X}$ PCR buffer $\left[100 \mathrm{mM} \mathrm{KCl}, 100 \mathrm{mM}\left(\mathrm{NH}_{4}\right)_{2} \mathrm{SO}_{4}, 200 \mathrm{mM}\right.$ Tris-HCl PH $8.3,1 \%$ Triton $X-100], 0.5 \mu \mathrm{L}$ of $10 \mathrm{mM}$ dNTPs, $2 \mu \mathrm{L}$ of $25 \mathrm{mM} \mathrm{MgCl}_{2}, 0.1 \mu \mathrm{L}$ of Taq DNA polymerase, and 9.4 $\mu \mathrm{L}$ of deionized water. PCR reactions were performed with the following reaction conditions: initial denaturation at $94^{\circ} \mathrm{C}$ for 1 minute, followed by 40 cycles at $94^{\circ} \mathrm{C}$ for 30 seconds, annealing for 30 seconds (annealing temperature varies depending on the different pairs of primers, typically $55-60^{\circ} \mathrm{C}$ ), and extension at $72^{\circ} \mathrm{C}$ for 30 seconds. These cycle parameters were followed by incubation at $72^{\circ} \mathrm{C}$ for 10 minutes, followed by $4^{\circ} \mathrm{C}$ for an indefinite time.

\subsubsection{Agarose gel electrophoresis conditions}

Gel electrophoresis was performed to analyze the PCR results. A $4 \%(w / v)$ agarose gel comprised of $1 \times$ TAE buffer and $0.05 \%$ ethidium bromide was used to electrophoresis all the PCR products. $5 \mu \mathrm{L}$ of $5 \mathrm{X}$ blue dye was added to each $20 \mu \mathrm{L}$ volume sample. Then, $13 \mu \mathrm{L}$ of mixed samples were loaded on to the gel. The agarose gel was run at 80 volts for about an hour. The gel bands were visualized using an Alphalmager 2200 gel-imager system (Alpha Innotech, San Leandro, CA). For 
amplification of genomic DNA fragments for seqencing, a $0.8 \%$ agarose gel was used, and run at 100 volts for about 20 minutes.

\subsubsection{Mutation site verification}

In order to verify the mutation sites in the CER6 gene of line8, PCR primers were designed to amplify fragments of the CER6 gene, based on the genomic DNA sequence obtained from TAIR (http://www.arabidopsis.org). Genomic annotation obtained from TAIR indicated that the length of the wild type CER6 coding region was 2220 bps (from 5'-UTR to 3'-UTR). Two pairs of primers were designed using the same strategy for designing primers for amplification of polymorphic markers: LP1 (5'GCAGCGATGAAAACAAA-3'), LP2 (5'-TGGTGATGTTTGCCTTGA-3'), RP1 (5'GGCAAACATCACCACAAT-3'), RP2 (5'-TTCCTCTGTTCGCCTTTA-3'). CER6 can be represented completely by the two PCR products (LP1+RP1 and LP2+RP2), which were each around 1200 bps. DNA fragments generated by PCR were purified using Wizard® Plus SV Minipreps DNA Purification System (Promega). The sequencing reactions were carried out at DNA Landmarks (Montreal, QC). The sequenced DNA fragments were assembled together using Seq Man II Lasergene software (DNASTAR, US).

\subsubsection{Toluidine-blue (TB) assay}

All the seeds were surface-sterilized with $50 \%$ bleach for $10 \mathrm{~min}$ and washed with $\mathrm{dH}_{2} \mathrm{O}$ 5-6 times. The sterilized seeds were sown on a $0.7 \%$ AT plate (Somerville and Ogren, 1982) and incubated at $4^{\circ} \mathrm{C}$ for $3-4$ days. All the plates were then moved to a Conviron chamber for 10 days. Each seedling was taken from the plate and soaked in the toluidine-blue (TB) solution for $2 \mathrm{~min}$. TB solution is an aqueous solution of $0.05 \%$ 
TB (Fluka) filtered through a $0.2 \mu \mathrm{m}$ syringe filter (Acrodisc). Stained seedlings were washed gently with $\mathrm{dH}_{2} \mathrm{O}$ to remove excess TB from surfaces. The Zeiss Axio program was used to take pictures immediately following staining.

\subsubsection{Fresh water loss assay}

A fresh water loss assay was performed to analyze epidermal permeability. Stem and leaf samples were used from 4-5 week-old plants. Plants were kept in the dark for at least 4 hours prior to analysis. Detached-rosette leaves and stems with cauline leaves removed were soaked in water for $60 \mathrm{~min}$ in the dark. Rosettes and stems were shaken gently to remove excess water and kept in complete darkness to keep stomata closed. The tissues were weighed at regular intervals for 1 hour using a microbalance (TE2145, Sartoris). The rate of water loss per gram of fresh weight tissue was determined by the formula (initial weight - measured weight)/initial weight.

\subsubsection{Chlorophyll-leaching assay}

Chlorophll-leaching was performed using rosette leaves of 3-week old plants. The detached-rosette leaves were weighed and then immersed in $30 \mathrm{~mL}$ of $80 \%$ ethanol at room temperature in the dark. Test tubes with rosette and ethanol mixtures were shaken gently and $200 \mu \mathrm{L}$ aliquots of supernatant were transferred separately into different $1.5 \mathrm{~mL}$ tubes at $10,20,30,40,50,60,90,120$ minutes after starting. After all of the samples were taken, $100 \mu \mathrm{L}$ aliquots were transferred into a 96 microwell plate (Lot No. 05370115 , Greiner bio-one) from each $1.5 \mathrm{~mL}$ tube. The plate was read on a UV microwell spectrophotometer (Molecular Devices Spectra max 340 PC 384) at absorbances of $647 \mathrm{~nm}$ and $664 \mathrm{~nm}$. The total micromoles of chlorophyll leached per 
gram of fresh weight tissue were calculated using the equation: total micromoles of chlorophyll=7.93( $\left.\mathrm{A}_{664}\right)+19.53\left(\mathrm{~A}_{647}\right)($ Lolle et al., 1997$)$. 


\subsection{Results}

3.3.1 Rough mapping: linkage analysis of line 8 and line 85 to molecular markers.

A collection of polymorphic DNA markers were selected that span the five chromosomes (Figure 12). Some of these were taken from Lukowitz et al (2000) and some were designed by myself. The detailed information of these markers and primers for amplification by PCR are summarized in Table 5 .

Upon first-pass mapping of selected markers from Figure 12, LINE8 showed linkage to marker nga111 at the bottom of chromosome I, with a recombination frequency of $7.5 \%$ based on 50 samples; LINE85 showed linkage to marker CER449723 at the top of chromosome I, with a recombination frequency of $17.7 \%$ based on 50 samples (Figure 12). LINE8 had a recombination frequency of $40-55 \%$ with all of the other markers indicating that they are not linked. LINE85 had recombination frequencies of $51.8 \%$ and $44.7 \%$ to markers on the bottom of chromosome IV and V, respectively, indicating that these two markers were unlinked. 


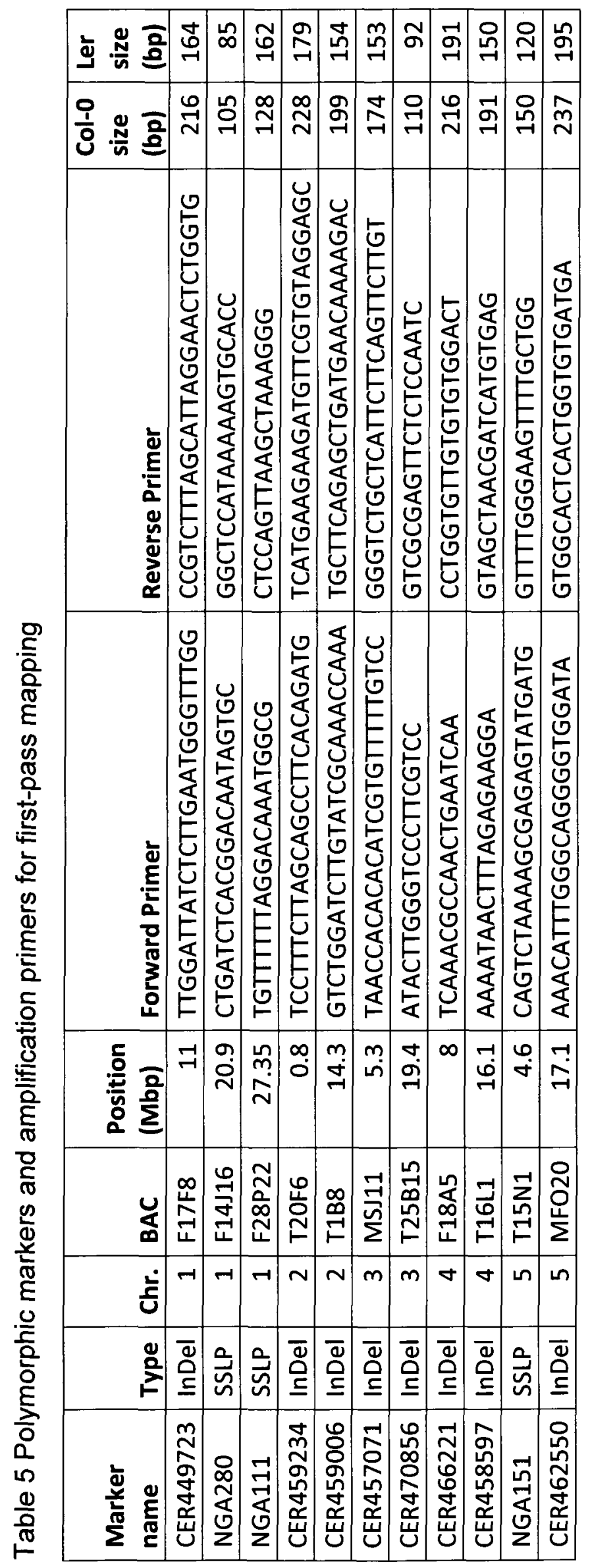




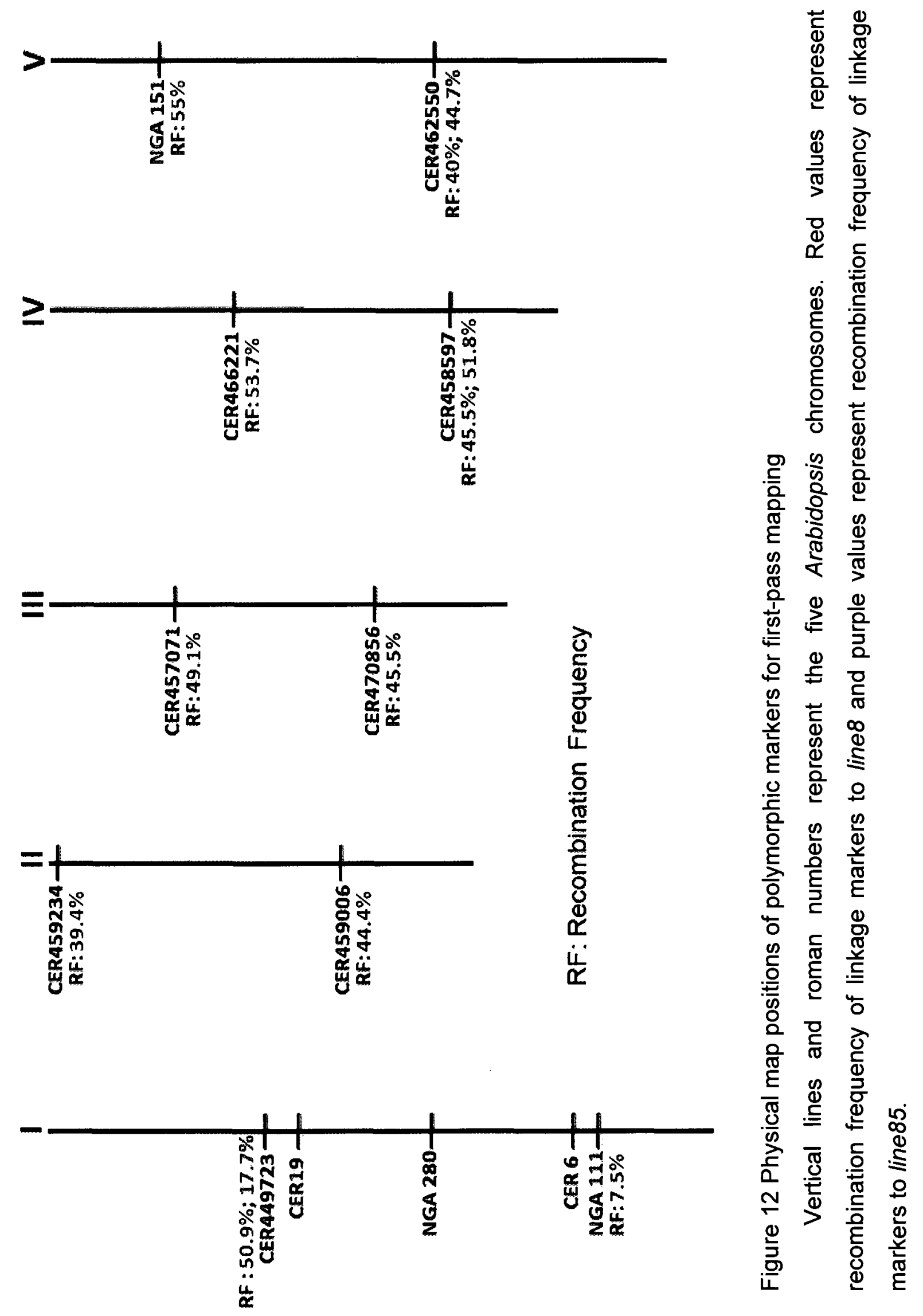


3.3.2 Comparisons of crystal morphology and wax composition between line8 and cer6.

First-pass mapping of line8 showed linkage to the marker NGA111 at position 27.35 Mbp on chromosome I. This position was physically close to the CER6 locus (Fiebig et al., 2000). Thus, a detailed comparison of the epicuticular wax crystal density and shape were conducted using SEM, and comparison of wax load and composition conducted by GC.

The wax crystals on the stem surfaces of line 8 were reduced in density, but not absent (Figure 13A). The shape of line8 crystals was very unique, being flattened and irregular in shape reminiscent of fishbones. This, in part, led us to speculate that line 8 was a unique loci affecting cuticular wax metabolism. The stem surfaces of cer6 mutants are devoid of crystals (Figure 13A).

However, inspection of the cuticular wax composition of line8 stems revealed much similarity with the reported cer6-1 phenotype (Jenks et al., 1995). Both line8 and cer6 have reductions in C28 and C30 free fatty acids and large increase in C24 and C26 free fatty acids (Figure 13B). Primary alcohols of chain lengths C24 and C26 are also significantly increased in both mutants. Aldehydes, alkanes, secondary alcohols, and ketones (alkane-forming pathway components) with chain lengths beyond C26 are reduced in both mutants, while chain lengths C26 and lower are increased. CER6 encodes a ketoacyl CoA synthase involved in fatty acid elongation, consistent with this phenotype (Millar et al., 1999; Fiebig et al., 2000). 
Figure 13 Comparison of wax crystal shape and cuticular wax composition between line8 and cer6

(A) Scanning electron microscopy images of line8, cer6 and the corresponding wild type ecotype. The SEM images taken at magnifications of 1500X, 5000X and 10000X, respectively. The scale bars are shown at the bottom of each panel.

(B) Stem cuticular wax composition of line8, cer6 and the corresponding wild-type ecotype (Col-0) sorted by chemical type.

All values are means, presented in $\mu \mathrm{g} / \mathrm{cm}^{2}$, derived from three replicates $(n=3)$. Error bars represent the standard deviation. 


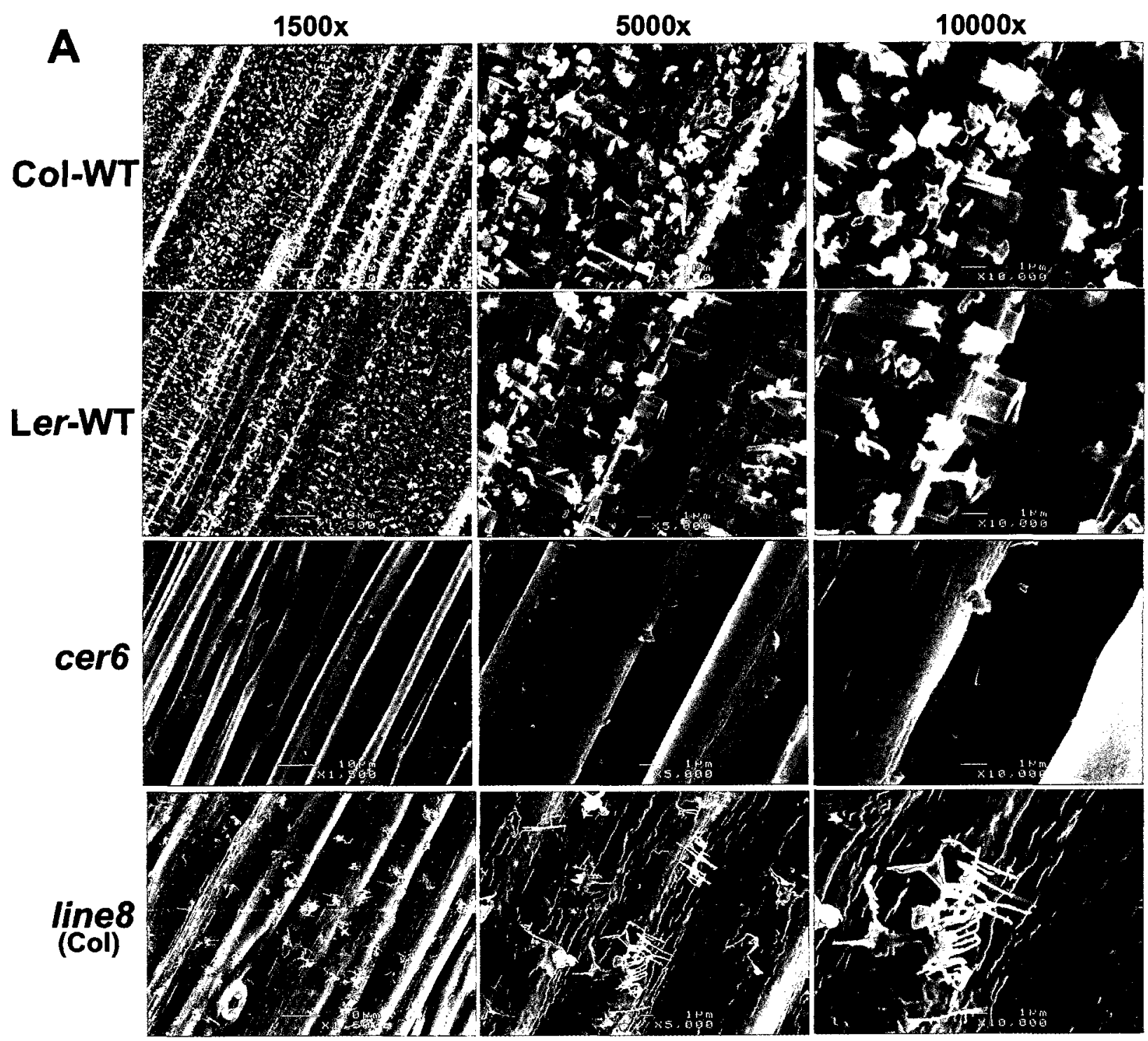




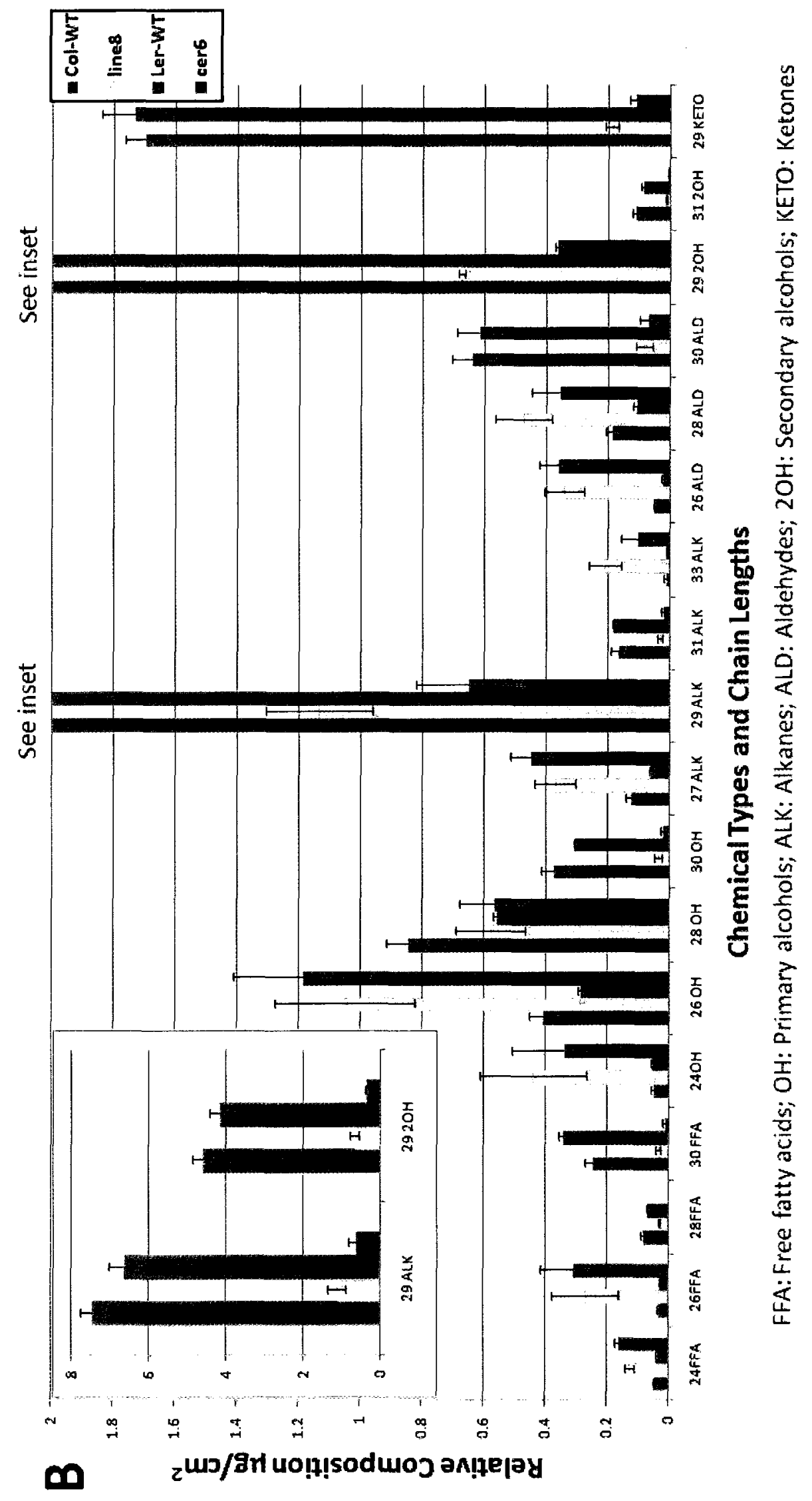


3.3.3 Sequencing of CER6 gene in the line8 mutant and alignment of predicted protein sequences

Since the wax composition of line 8 mutant is similar to that of cer6 and the two loci are linked, it was possible that line 8 was an unusual allele of CER6. The CER6 gene was sequenced in the line 8 mutant to test for the presence of a mutation in order to confirm that line 8 is indeed an allele of cer6. The CER6 gene in line 8 was found to have a one base pair substitution at $1190 \mathrm{bp}$ position that changed position 397 alanine, a small hydrophobic amino acid, to valine, a larger hydrophobic amino acid (Figure 14).

The amino acid sequence of CER6(A397V) was compared with wild-type CER6 (At1g68530), KCS17 (DAISY, At1g04220), and FAE1 (KCS18, At4g34520), three genes from Arabidopsis that are highly expressed in epidermis, roots, and seeds, respectively (Millar et al., 1999; Rossak et al., 2001; Franke et al., 2009). Two KCSs from other species, StKCS6 from Solanum tuberosum (potato) (Serra et al., 2008) and LfKCS45 from Lesquerella fendleri (Moon et al., 2005), were also included. Comparison of sequence alignment revealed the presence of six conserved cysteine residues and four histidines (Figure 14), which are important for enzyme activity (Ghanevati and Jaworski, 2001). A397 was highly conserved in all the aligned putative KCSs. Moreover, the A397V mutation site is in close proximity to the conserved cysteine residue at position 390.

\subsubsection{Complementation crosses between line8 and cer6}

To test for allelilism between line 8 and cer6, reciprocal complementation crosses between line 8 and cer6 were performed. The glossy phenotype in the F1 generation from complementation crosses confirmed that line 8 is allelic to cer6 (Figure 15). We 
therefore re-name line8 as cer6-3, continuing from the allelic numbering described in Fiebig et al (2000) for cer6 mutants. 
Figure 14 Amino acid sequence comparison of CER6(A397V), CER6(WT), KCS17, FAE1, LfKCS45, and StKCS6

GenBank accession numbers of the sequences used are: Arabidopsis CER6(WT), NP_177020; Arabidopsis KCS17, NP_171918; Arabidopsis FAE1, Q38860; Lesquerella fendleri LfKCS45, AAU05611; potato StKCS6, ACF17125. Identical residues are highlighted on a black background; similar amino acids are shown on a gray background. Conserved cysteine and histidine residues are labeled with red triangles and green diamonds, respectively. The mutation site of CER6(A397V) is labeled on a yellow background. 


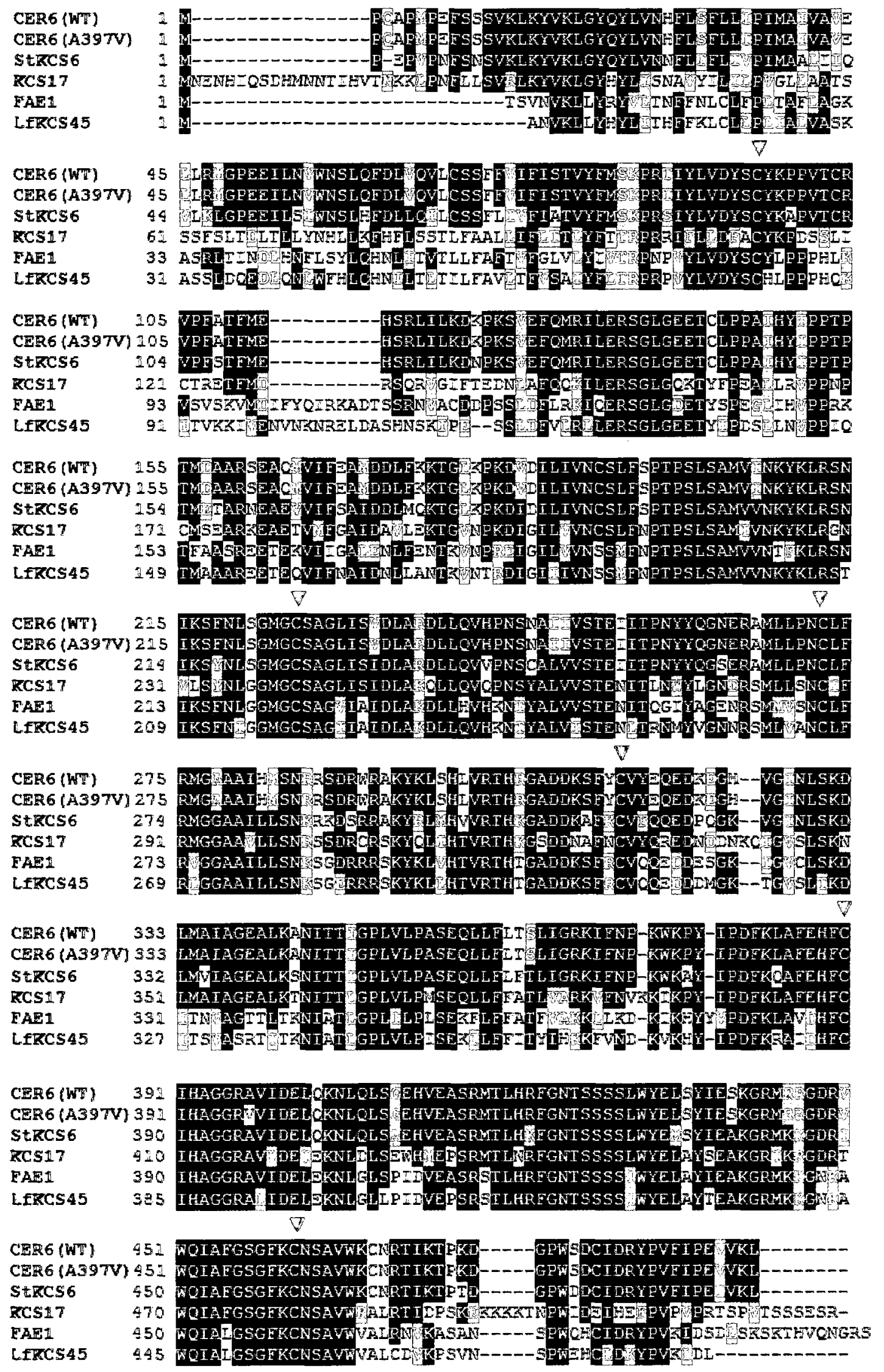




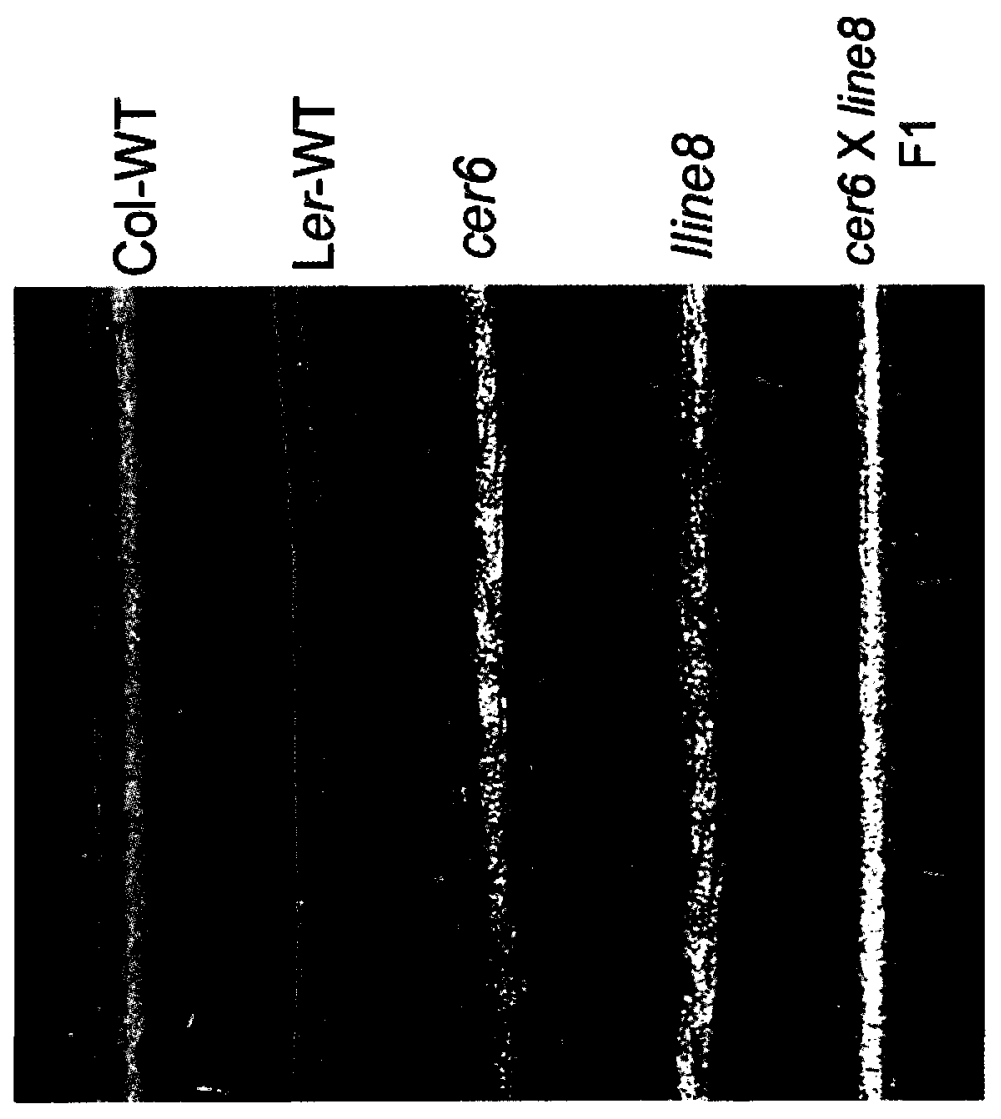

Figure 15 Stem phenotypes of wild-type, cer6 and F1 plants from complementation crosses between line8 and cer6.

The stems from left to right are Col-WT, Ler-WT, cer6, line8, and F1 of cer6 $\mathrm{X}$ line8. 
3.3.5 Comparisons of crystal morphology and wax composition between line85 and cer19.

First-pass mapping of line85 showed linkage to the marker CER449723 at position 11.0 Mbp on chromosome I. This position was physically close to the reported CER19 locus (Rashotte et al., 2004), but the gene has not been cloned yet. Thus, a detailed comparison of the epicuticular wax crystal density and shape was conducted using SEM, and comparison of wax load and composition conducted by GC.

The wax crystals on the stem surfaces of line 85 were similar in shape to crystals on the stem surface of cer19 (Figure 16A). However, the density of crystals was significantly lower on line 85 compared to cer19 causing us to initially think that they are phenotypically distinct and representing different loci.

However, perturbations of cuticular wax composition in line85 and cer19 were nearly identical (Figure 16B). Both mutants have dramatically reduced levels of wax components that are $\mathrm{C} 29$ or higher, which is anything ultimately derived from $\mathrm{C} 30$ or longer fatty acyl-CoA precursors. Both the primary alcohol and alkane forming pathways were affected. C24 and C26 primary alcohols and C27 alkanes are both increased in line 85 and cer19 compared to wild-type.

\subsubsection{Complementation crosses between line85 and cer19}

The close proximity of the line85 and cer19 loci and similar wax composition defects suggested that they are in fact allelic. To test for allelism between the two mutants, reciprocal complementation crosses between line85 and cer19 were performed. The glossy phenotype in the F1 generation from complementation crosses confirmed that line85 is allelic to cer19 (Figure 17). Both mutants are recessive alleles 
(data not shown; Koornneef et al., 1989). We therefore re-name line85 as cer19-2, with the original cer19 mutant identified by Koornneef et al as cer19-1. 
Figure 16 Comparison of wax crystal shape and cuticular wax composition between line85 and cer19

(A) Scanning electron microscopy images of line85, cer19 and the corresponding wild type ecotype (Ler-WT). The SEM images were taken at magnifications of 1500X, 5000X, and $10000 \mathrm{X}$, respectively. The scale bars are shown at the bottom of each panel.

(B) Stem cuticular wax composition of line85, cer19 and corresponding wild-type ecotype (Ler-WT) sorted by chemical type.

All values are means, presented in $\mu \mathrm{g} / \mathrm{cm}^{2}$, derived from three replicates $(n=3)$. Error bars represent the standard deviation. 


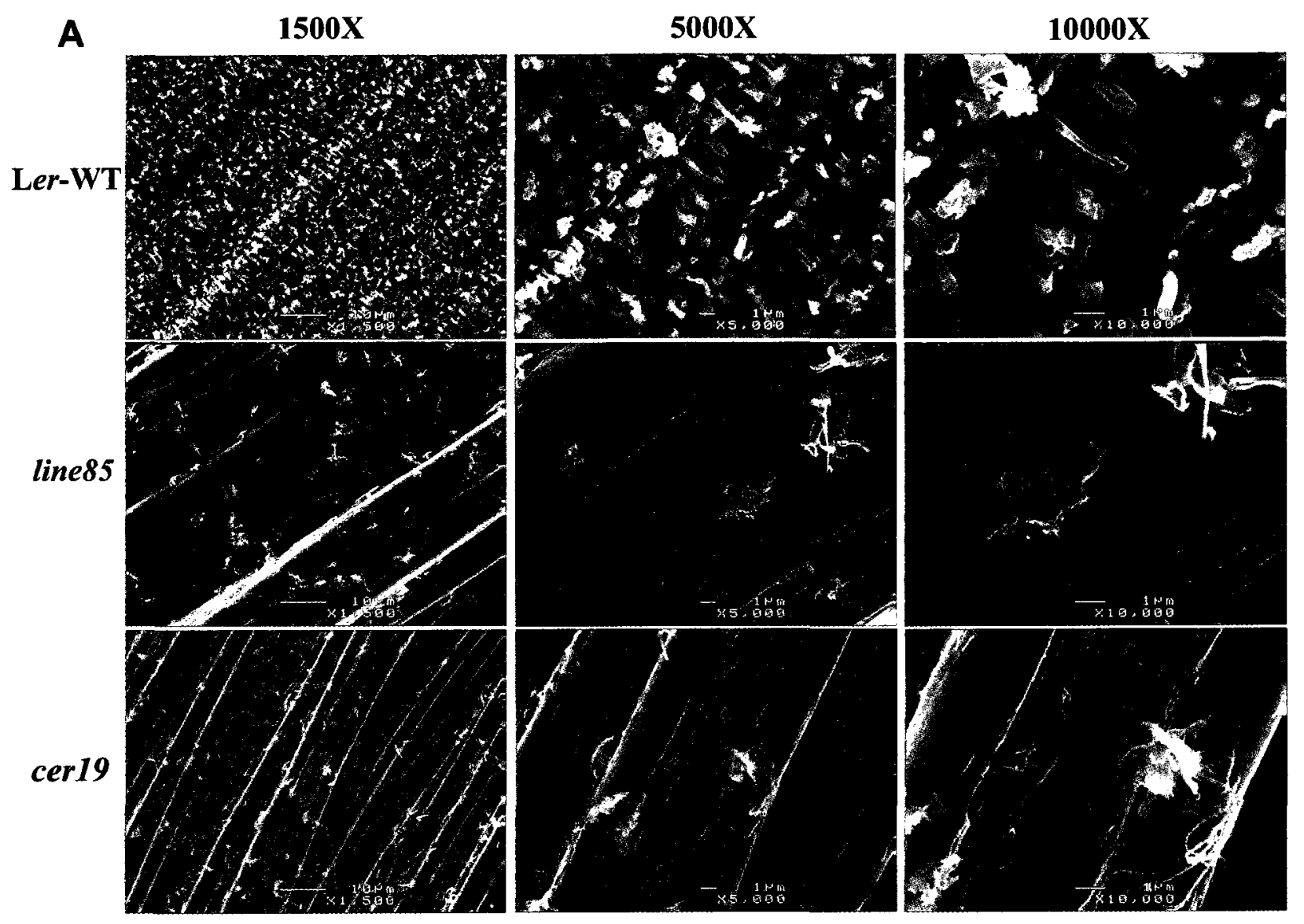




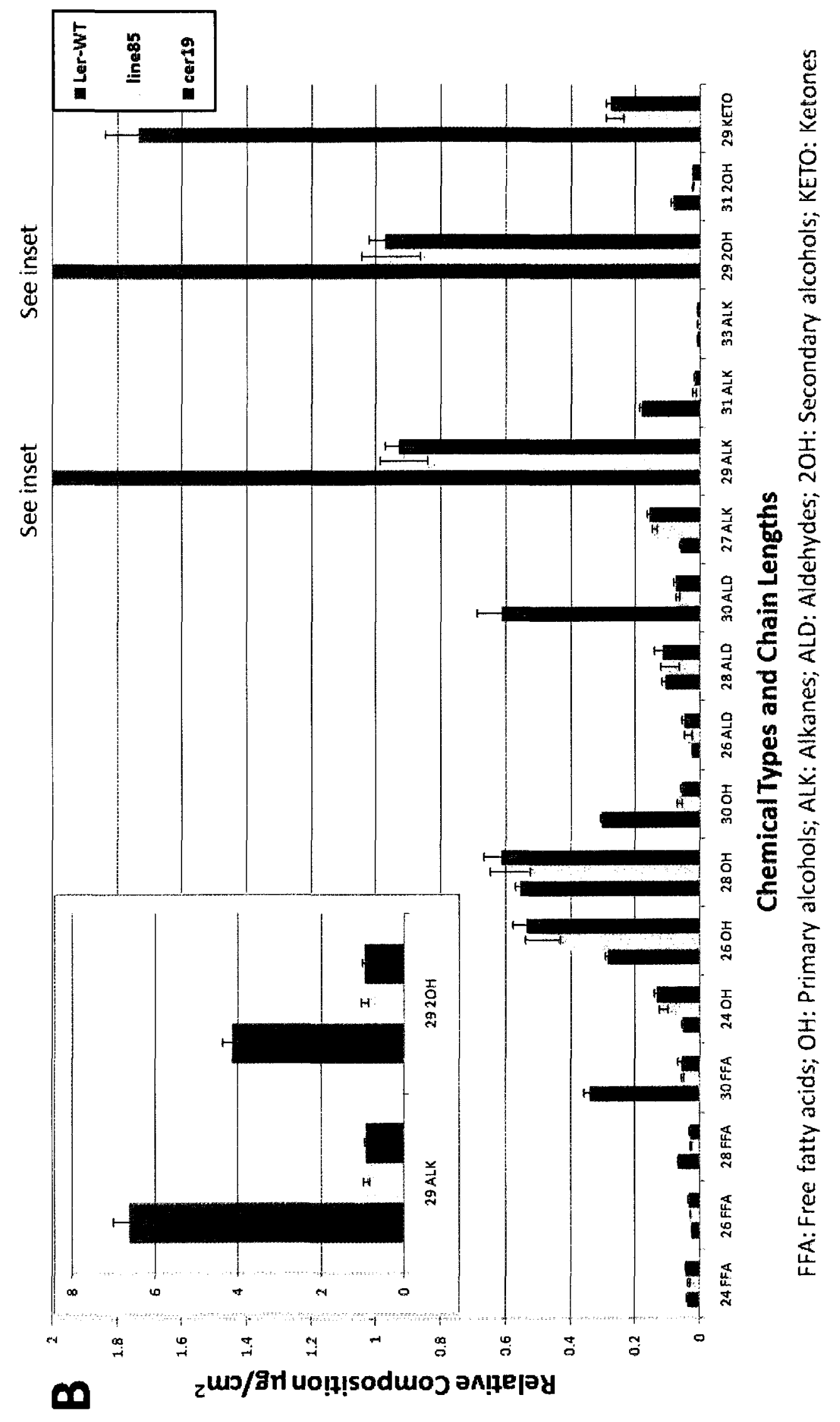




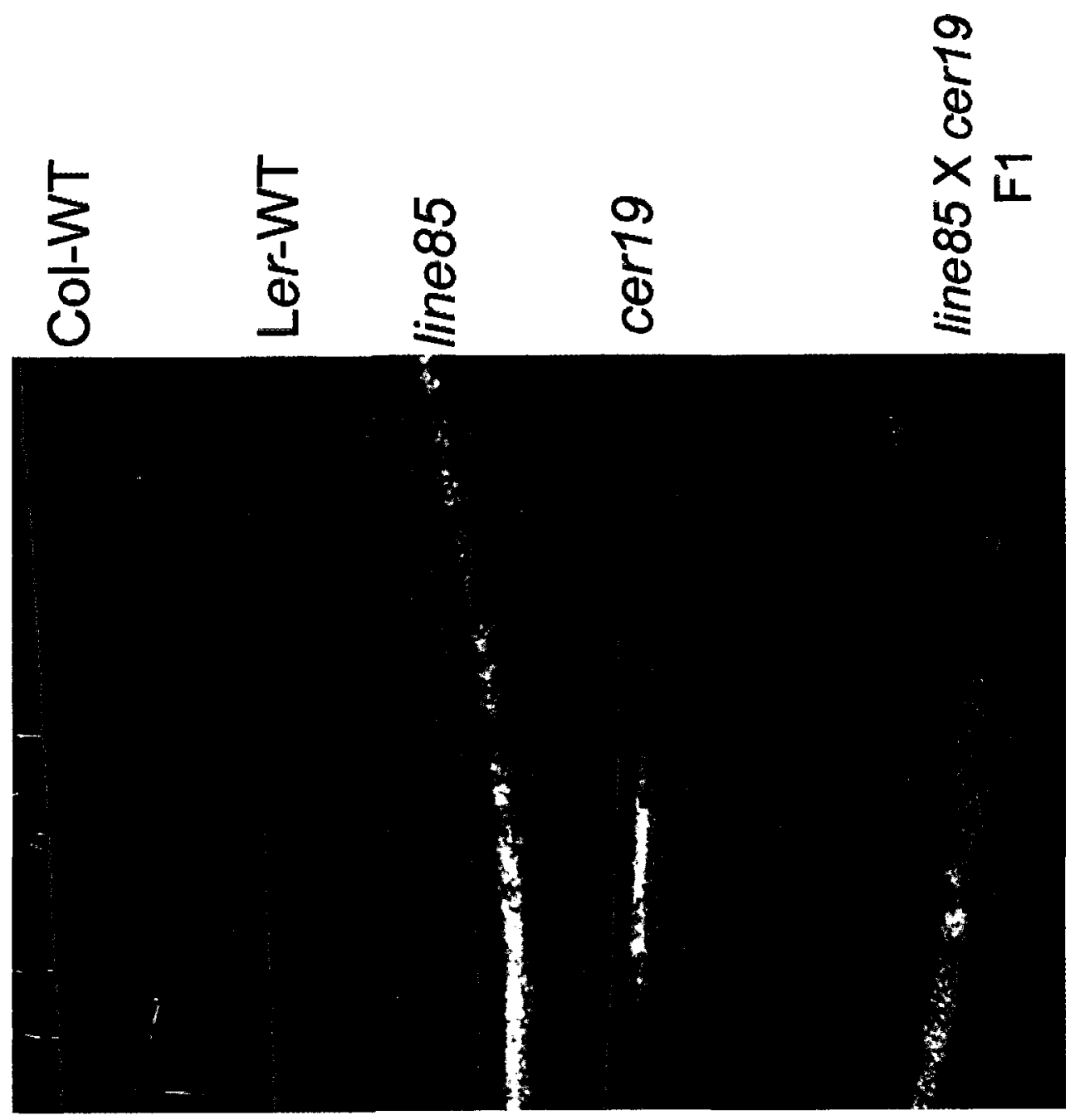

Figure 17 Stem phenotypes of wild-type, line85, cer19 and F1 plants from complementation crosses between line85 and cer19.

The stems from left to right are Col-WT, Ler-WT, line85, cer19, and F1 of line85 X cer19. 


\subsubsection{Cuticular permeability analysis}

We extended the phenotypic analysis of cer19-1 and cer19-2 (line85) by measuring effects on cuticular permeability. Three measurements of cuticular permeability were performed: 1) toluidine blue (TB) assay (Figure 18), 2) rate of water loss from fresh tissue (Figure 19), and 3) rate of chlorophyll-leaching from fresh tissue (Figure 20).

The staining by TB was significantly increased in cer19-1 and cer19-2 in 10 day old seedlings compared to wile-type (Figure 18). This is indicative of a continuous defect in the developing cuticle of these seedlings. This is a class I staining pattern according to Tanaka et al (2003), whereas, mutants of other classes (II to V) have patchy, proximalonly, distal-only, or trichome-only staining patterns. There were no differences in the TB staining of adult plants between the cer19 mutants and wild-type (data not shown).

The water loss assay measures the diffusion of non-stomatal water loss from plant tissues to the environment. The rosette leaves of line85 and cer19 both showed a slightly increased rate of water loss over time compared to wild-type (Figure 19A). But the error bars are huge, so the results may not be statistically significant. While stems showed similar rates of water loss between mutant and wild-type (Figure 19B).

A chlorophyll-leaching test of cer19-1, cer19-2, and Ler-WT plants was also performed as an additional measurement of cuticular permeability in adult plants. The detached rosette leaves of both cer19-1 and cer19-2 exhibited increased chlorophyll leaching in $80 \%$ ethanol compared to wild-type ecotype, especially apparent at 60 minutes (Figure 20). At two hours following immersion of tissue in $80 \%$ ethanol, the concentration of chlorophyll leached from cer19-1 and cer19-2 rosette leaves was 2-3 
times greater than that of rosette leaves from Ler-WT plants. According to T-test statistical analysis, with $d f=8, t$ must be at least 2.5 to reach $p$-value $<0.05$. Our result was $t_{\text {line85 }}=1.9225 ; t_{c e r 19}=1.8932$, so by conventional criteria, this difference is considered not to be statistically significant. 

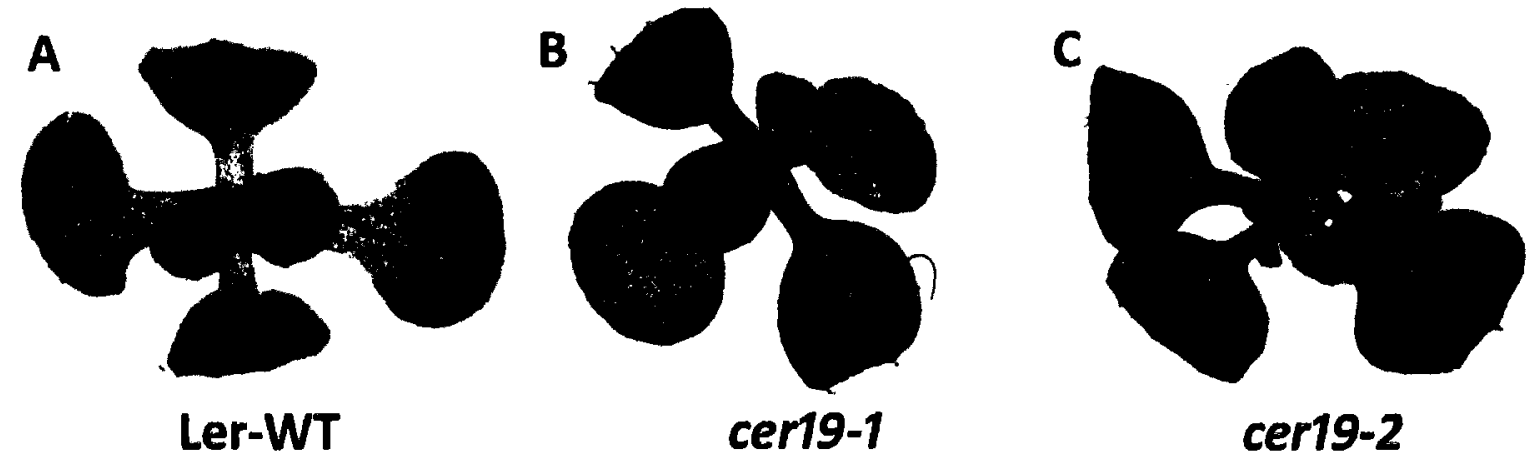

Figure 18 Toluidine blue staining of Ler-WT, line 85, and cer19 seedlings TB stain on leaves of 10 day old seedlings of (A) Ler-WT, (B) cer19-1, and (C) cer19-2

92 
Figure 19 Rate of water loss in fresh tissue of cer19-1, cer19-2, and Ler-WT (A) Fresh weight assay with leaves of 5-week old cer19-1, cer19-2, and Ler-WT plants, where the rate of water loss per gram of fresh weight tissue from the rosette leaves was measured at the indicated time points $(n=5 ; \pm$ standard deviation). The plants were kept in the dark for at least four hours prior to measurement to make sure stomata were closed.

(B) Fresh weight assay with stems of 5-week old cer19-1, cer19-2, and Ler-WT plants, where the rate of water loss per gram of fresh weight tissue from stems was measured at the indicated time points $(n=5 ; \pm$ standard deviation). The plants were kept in the dark for at least four hours prior to measurement to make sure stomata were closed. 

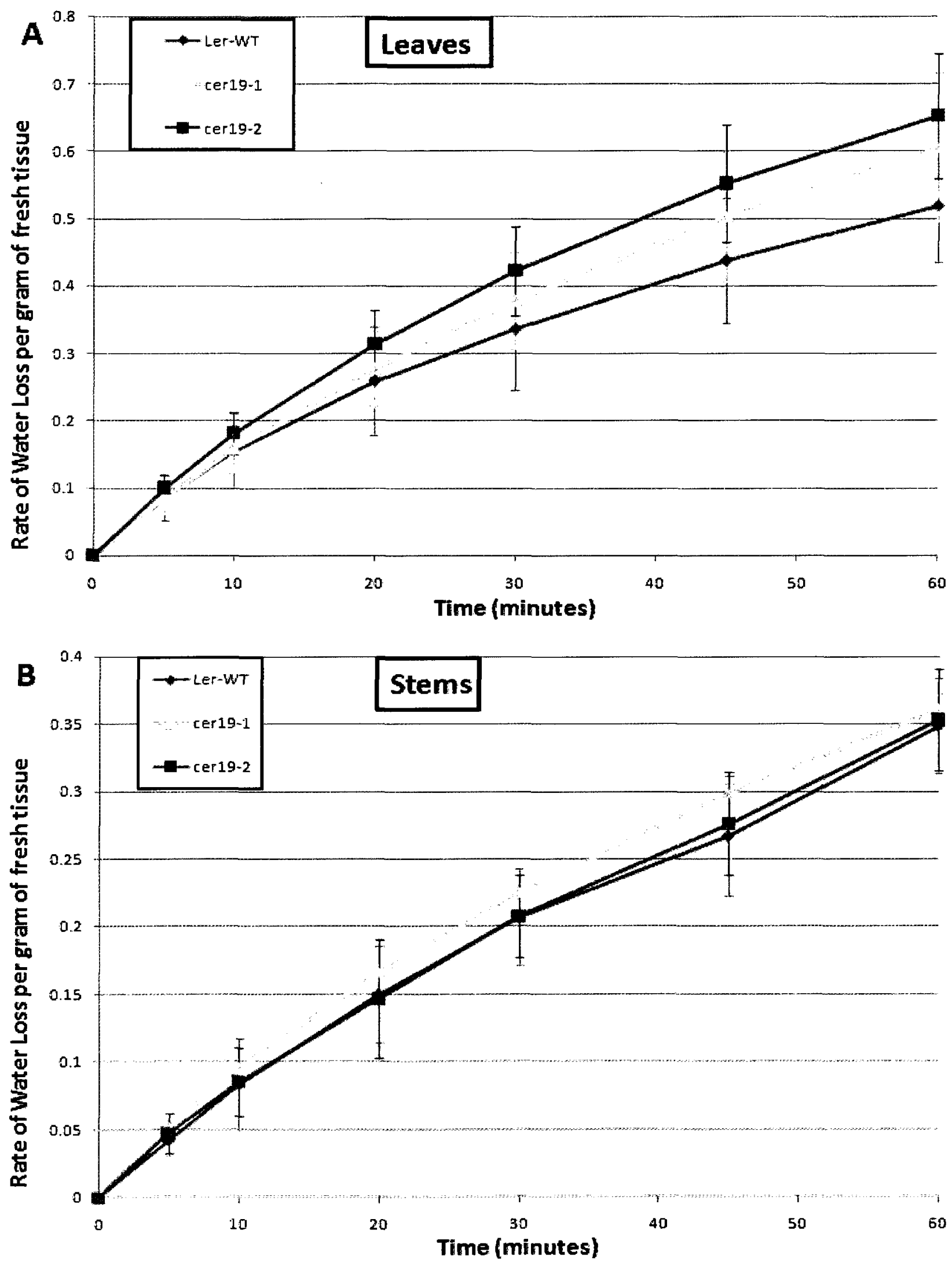


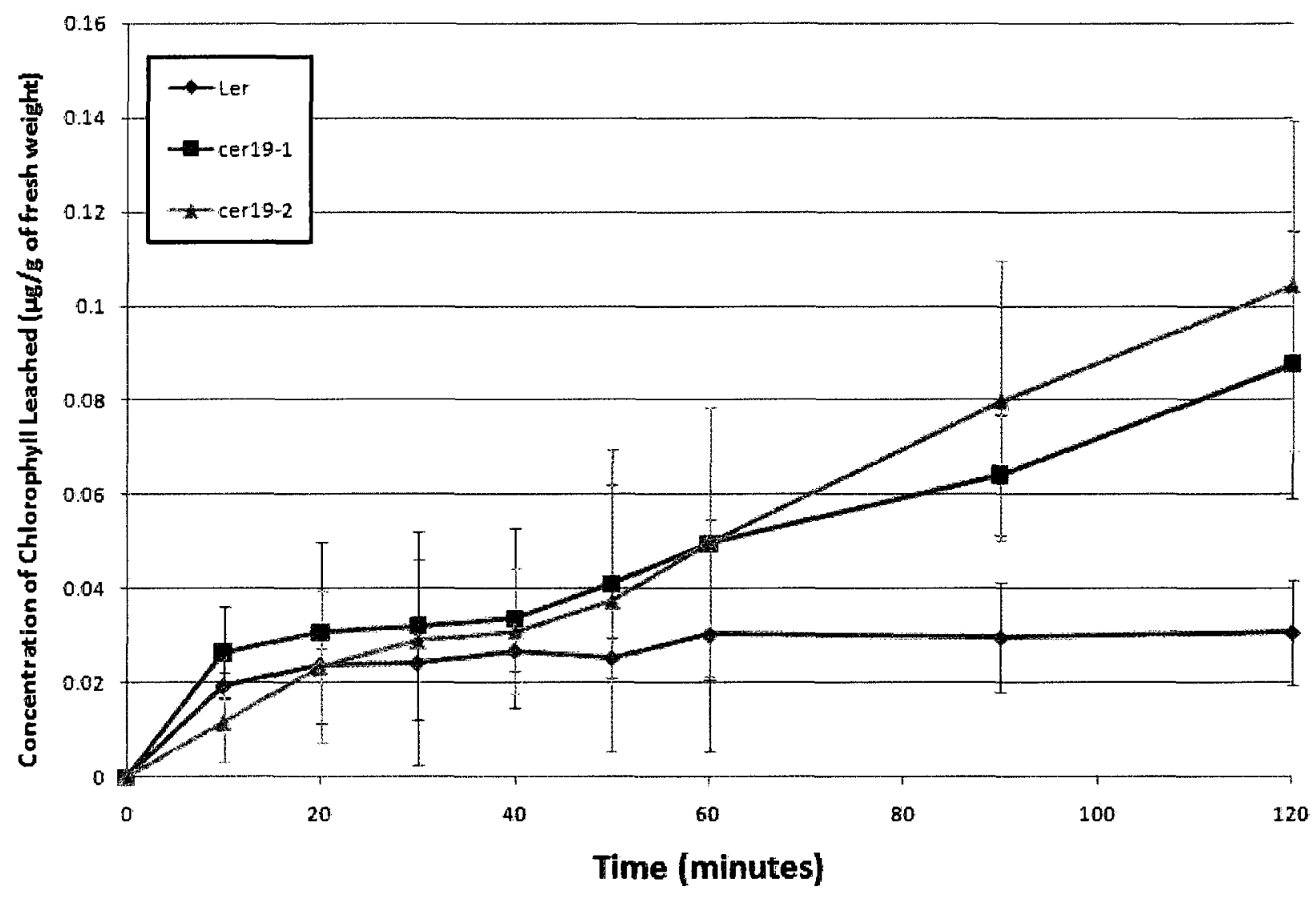

Figure 20 Chlorophyll-leaching assay of cer19-1, cer19-2, and Ler-WT leaves.

The concentration of chlorophyll leached over 2 hours into a solution of $80 \%$ ethanol from rosette leaves taken from 4 week old plants of cer19-1, cer19-2, and LerWT ( $n=10 ; \pm$ standard deviation). 


\subsection{Discussion}

I originally speculated that the mutants line 8 and line 85 represented novel loci based on their unique defects in wax crystal formation and cuticular wax composition (Chapter 2). To test this, I first roughly mapped their respective genetic positions by linkage analysis to molecular markers spread out over the five Arabidopsis chromosomes. LINE8 mapped near to the CER6 locus and LINE85 mapped near to the CER19 locus. In addition, closer inspection of the cuticular wax defects revealed phenotypic relationships between the respective mutants. Complementation tests confirmed that line 8 is allelic to cer6 and this was confirmed by the presence of a nonsilent point mutation in the CER6 gene present in the line 8 background. We therefore renamed line 8 as cer6-3. In addition, complementation tests confirmed that line 85 is allelic to cer19, and line85 was renamed cer19-2.

CER6 encodes a $\beta$-ketoacyl-CoA synthase (KCS) or condensing enzyme involved in fatty acid elongation of fatty acyl-CoAs longer than C26 in epidermal cells (Millar et al., 1999; Fiebig et al., 2000; Hooker et al., 2002). There was a low density of wax crystals that accumulated on the stem surfaces of cer6-3, while wax crystals were not detectable on the stem surfaces of cer6-1. It is likely that cer6-3 is a weaker allele than cer6-1, accounting for this difference in phenotype. The mutation in the CER6 gene of cer6-3 is a one-base pair substitution at nucleotide $1190 \mathrm{bp}$, relative to the start codon, changing amino acid residue 397 from alanine to valine. This amino acid is near to one of the active-site residues necessary for enzymatic activity, but is not thought to be directly involved in catalysis (Ghanevati and Jaworski, 2000; Joubes et al., 2008). Also, the change from a small hydrophobic residue (alanine) to a larger one (valine) is relatively 
conservative and thus activity of the enzyme may not be completely knocked out in cer6-3. Consistent with this hypothesis, the C29 alkanes, C29 secondary alcohols, and C29 ketones, which make up the majority of stem cuticular wax were more reduced in cer6-1 than cer6-3. The lesion in cer6-1 is a three-base deletion eliminating codon 319 of the CER6 coding region and is thought to be a null mutation (Fiebig et al., 2000). Fiebig et al., 2000 also characterized the cer6-2 allele and found that it contained an Ato- $C$ transversion in the CER 6 gene that changes the histidine at amino acid position 148 to proline. In addition, this study reported an intragenic suppressor of cer6-2 called cer6-2R that has a C-to-T transition at this same codon converting it to a serine in the encoded protein. This is a partial suppressor and there is only slight restoration of wax crystals relative to cer6-2 stems. The density and shape of the wax crystals on cer6-2R are very similar to the density and shape of crystals on cer6-3, again consistent with cer6-3 being a relatively weaker allele than the null mutation.

The CER19 gene has not yet been cloned and thus molecular characterization of the two cer19 alleles is not yet possible. The cer19-2 appears to be a stronger allele than cer19-1, due to the more dramatic reduction of wax crystals in cer19-2. However, the wax load and composition was not significantly different between the two alleles. It is possible that there was subtle difference in wax production that could affect the amount of wax crystals formed. Alternatively, a background mutation in either of these alleles could be modifying the phenotype. Neither mutant has been backcrossed to eliminate backgrounds, which is important for eliminating background mutations that are not tightly linked. 
We performed three different physiological assays to test whether the cer19 mutants were significantly altered in cuticular permeability. Both alleles showed high permeability to TB stain compared to wild-type, but only in young seedlings. It is possible that the seedlings, which have a developing cuticle, are more sensitive to perturbations in cuticle composition than adult plants. The other two physiological assays, water loss and chlorophyll-leaching assays, revealed slightly increased cuticular permeabilities in tissues of adult cer19 mutants compared to wild type. However, there was considerable variation and it is uncertain if these differences are significant. Further replicates are needed to more conclusively investigate the cuticle permeability in cer19 mutants. Investigation of cuticle structure by transmission electron microscopy (TEM) may also reveal differences between cer19 and wild-type (Lu et al., 2009). However, studies have pointed out that cuticle permeability is largely determined by the nanomolecular structure and packing arrangement of cutin and wax molecules within the cuticle membrane itself (Reynhardt, 1997; Schreiber et al., 1997), and thus TEM analysis may not reveal microscale pertubations.

The cloning of CER19 is key to further understand its role in wax biosynthesis. The rough map position reported by Rashotte et al. (2001), which was further refined here, is an important starting point for this endeavor. The identification of cer19-2 as a second allele of cer19 will facilitate the identification of the CER19. For example, the two cer19 alleles are expected to have distinct mutations in the CER19 gene and this is good evidence of identification. The wax composition defect of cer19 suggests that the CER19 protein is involved in fatty acid elongation from C28 to C30 (Rashotte et al., 2001). There were significant decreases in all wax components derived from fatty acyl 
chains of C30 or higher. Wax components of chain lengths C28 or lower were either not affected or were increased. A block in fatty acid elongation at the C28 to C30 step would be expected to yield increases in the smaller intermediates. This type of accumulation is observed in cer6 mutants that are apparently blocked in fatty acid elongation at the $\mathrm{C} 26$ to $\mathrm{C} 28$ step. Therefore, it is possible that CER19 encodes for a KCS and there are candidate KCSs on the top of chromosome I. However, none of these have thus far been implicated in cuticular wax metabolism, at least as single mutations. A knockout of KCS2/DAISY (At1g04220), which is located at the top of chromosome I, does not affect wax accumulation, but a double mutation of kcs2 with $k c s 20$ does moderately reduce wax load (Lee et al., 2009b). The transcripts of KCS1 (At1g01120) and KCS3 (At1g07720) are enriched in the epidermis (Suh et al., 2005), indicative of roles in cuticular wax metabolism, but they are relatively far away from the mapped CER19 locus. KCS5 (At1g25450) is much closer to the CER19 locus and its mRNA is also enriched in the epidermis. Thus, KCS5 is a good candidate for CER19. It is also possible that CER19 is a regulator that influences the $\mathrm{C} 28$ to $\mathrm{C} 30$ elongation step by modifying the expression, stability or activity of a KCS involved in this elongation step. It is more difficult to identify candidate genes for such a regulator. 


\section{Chapter 4 Characterization of cer9 Phenotype and Mapping of the CER9 Loci}

\subsection{Introduction}

The cer9-1 mutant was identified by Koornneef et al. (1989) and mapped to the lower portion of chromosome IV between $59.8 \mathrm{cM}$ and $76.0 \mathrm{cM}$. cer9-1 was derived from ethyl methanesulfonate (EMS) mutagenized plants in the Ler ecotype and found to be a recessive mutation (Koornneef et al., 1989).

Scanning electron microscopy analysis revealed that the wax density of cer9-1 is significantly reduced and morphologically altered compared to wild-type (Koornneef et al., 1989). The mutant is characterized by decreased tubule-like wax structures and altered conformation of the platelets (Koornneef et al., 1989). Jenks et al. (1995) analyzed the leaf wax composition and found that $\mathrm{C} 24: 0$ and $\mathrm{C} 26: 0$ free fatty acids are significantly increased (23-fold and 27-fold, respectively) compared to Ler-WT, while free fatty acids with chain lengths longer than C26 were not detectable. There are also large reductions in the alkane and primary alcohol components of all chain lengths. Conversely, C26 and C28 primary alcohol amounts in cuticular wax of cer9-1 stems are highly increased (more than two times), while C30 primary alcohols are decreased. All components in alkane forming pathway of cer9-1 stems are significantly reduced including all chain lengths. Thus, there are tissue-specific differences in cuticular wax composition tissues in cer9-1. In stems, it is possible that the alkane forming pathway in cer9-1 stems is blocked, which causes $\mathrm{C} 26$ and C28 fatty acyl-CoAs to be more available for acyl reduction, generating more primary alcohols. The production or availability of $\mathrm{C} 30$ acyl-CoAs may be affected in cer9, affecting both pathways. Cer9-1 seems to be affected in a key upstream part of wax metabolism, between elongation 
and enzymatic modification of elongated chains. Therefore, cloning and characterization of CER9 may be essential to elucidate this part of wax biosynthesis.

The objective of this chapter was to further characterize the defects of cer9-1 to gain further insights into CER9 function, and to clone the CER9 gene using a mapbased cloning strategy. 
4.2 Materials and Methods

4.2.1 Plant materials and growth conditions

Cer9-1 and wild type (Ler ecotype) were grown in a semi-controlled growth room (Nesbitt Biology Building, Room 113A) with a continuous light photoperiod and at a temperature of approximately $22^{\circ} \mathrm{C}$, which was controlled by a Mitsubishi air conditioner. 4.2.2 Mapping population generation

Cer9-1 (Koorneef et al., 1989), which is in Ler ecotype, was crossed with Col-0 wild-type ecotype using cer9-1 as the female and Col-0 as the male. All seeds were collected separately from each successfully developed silique derived from a cross and sown out to grow the F1 generation. The F2 generation seeds were collected separately from individual selfed F1 plants. Homozygous cer9-1 in the F2 mapping population were identified by their visible glossy stem phenotype. These plants were genotyped for genetic mapping of the CER9 locus. Genomic DNA extractions and PCR analysis were performed as described in Chapter 3.

\subsubsection{Phenotypic characterization of cer9-1}

Scanning electron microscopy and gas chromatography were performed as described in Chapter 2. Measurements of cuticular permeability, including fresh water loss assay, chlorophyll-leaching assay, and toluidine-blue staining, were performed as described in Chapter 3.

\subsubsection{Reverse-transcription PCR (RT-PCR)}

Reverse-transcription PCR (RT-PCR) analysis was used to compare the expression levels of genes known to be involved in wax and cutin biosynthesis in the Ler-WT and cer9-1 mutant. Total RNA from rosette leaves and stems were isolated 
from Ler-WT and cer9-1 using the TRIzol protocol (Invitrogen), according to the manufacturer's instructions. The RNA concentrations were quantified using a Nanodrop1000 spectrophotometer (Thermo Fisher Scientific, Nepean, ON). One $\mu \mathrm{g}$ of RNA was reverse transcribed into $\mathrm{cDNA}$ using ImProm-II ${ }^{\mathrm{TM}}$ Reverse Transcription System (Promega, Madison, USA), according to the manufacturer's protocol. First, the RNA was denatured at $68^{\circ} \mathrm{C}$ for 5 min to remove secondary structure. Then the RNA was snap cooled on ice. Each $15 \mu$ reaction mixture, containing $4 \mu \mathrm{l}$ of ImProm- $\|^{\mathrm{TM}} 5 \mathrm{X}$ reaction buffer (Promega), $1.6 \mu$ l of $25 \mathrm{mM} \mathrm{MgCl}_{2}, 1 \mu \mathrm{l}$ of $10 \mathrm{mM}$ dNTP, $1 \mu$ of $30 \mu \mathrm{M}$ oligo(dT), $0.25 \mu$ l of ImProm-II ${ }^{\mathrm{TM}}$ reverse transcriptase (Promega) and $13 \mu \mathrm{l}$ RNase-free water, was added directly to the RNA. The mixture was incubated at $50^{\circ} \mathrm{C}$ for $60 \mathrm{~min}$, and then the cDNA stock was diluted (1:1) for PCR amplification. The PCR reaction in a $20 \mu$ l final volume contained $2 \mu \mathrm{l}$ of diluted cDNA, $1 \mu$ l of $10 \mathrm{mM}$ reverse primer, $1 \mu \mathrm{l}$ of $10 \mathrm{mM}$ forward primer, $2 \mu \mathrm{l}$ of $10 \mathrm{X}$ PCR buffer, $1.6 \mu \mathrm{l}$ of $25 \mathrm{mM} \mathrm{MgCl} 2,0.1 \mu$ l of Taq DNA polymerase, and $11.7 \mu \mathrm{l}$ of deionized water. The sequences of the gene specific primers used are listed in Table 6 . The PCR reaction was started with an initial step at $94^{\circ} \mathrm{C}$ for 1 min, followed by 28 cycles at $94^{\circ}$ for 30 seconds, annealing at $57^{\circ} \mathrm{C}$ for 30 seconds, and extension at $72^{\circ} \mathrm{C}$ for 30 seconds. These cycle parameters were followed by incubation at $72^{\circ} \mathrm{C}$ for $10 \mathrm{~min}$, followed by $4^{\circ} \mathrm{C}$ for an indefinite time. All the PCR products were seperated on a $0.8 \%(\mathrm{w} / \mathrm{v})$ agarose gel by electrophoresis with ethidium bromide as DNA intercalating agent for fluorescent detection. The gel image was photographed using an Alphalmager 2200 gel-imager system (Alpha Innotech, San Leandro, CA). 
Table 6 Gene-specific primers used in RT-PCR

\begin{tabular}{|l|l|}
\hline Primer & Sequence \\
\hline CER1-LP & 5'-AGATAAGAAAGGAGTGAAGGTGCTT-3' \\
\hline CER1-RP & 5'-AGAGATTTTGGAACTATCAATGCTG-3' \\
\hline CER2-LP & 5'-CTACTCCAATCTTATCACCACGTCT-3' \\
\hline CER2-RP & 5'-CTATCAATGTGGTTGAGGCTAAAAT-3' \\
\hline CER3-LP & 5'-TCCAATACTTCTTACCGTTTGCTAC-3' \\
\hline CER3-RP & 5'-CGTAAGTGCAGTTCCTTCTAAACTT-3' \\
\hline CER4-LP & 5'-GAAGTATGGTCCCAATCTAAATCAA-3' \\
\hline CER4-RP & 5'-ATTTTCTCTTTTTGTCCCTACCATC-3' \\
\hline CER5-LP & 5'-AAGAGAGATTGAGTGGTTACTACGG-3' \\
\hline CER5-RP & 5'-TGATAAGTCCCACCACTTAGAATGT-3' \\
\hline CER6-LP & 5'-CCTAATTACTATCAAGGCAACGAGA-3' \\
\hline CER6-RP & 5'-ATGTAGCTAAGCTCGTACCATAACG -3' \\
\hline CER8-LP & 5'-GATTTTCTCCATATGGGACGTG -3' \\
\hline CER8-RP & 5'-CGCGACAAGCATAGATTTGA-3' \\
\hline LACS2-LP & 5'-GTAATCTTAAACAACGCAGCAATTT-3' \\
\hline LACS2-RP & 5'-AACTCTCAGTCAATCCATAACCTTG-3' \\
\hline ATT1-LP & 5'-ACAACTACCCTAAAGGTCCTACGTG-3' \\
\hline ATT1-RP & 5'-CAGCAATTCTTGCTTACGTGTATTA-3' \\
\hline KCS1-LP & 5'-CGAAGTCGGAATCTTGATAGTAAAC-3' \\
\hline KCS1-RP & 5'-GTCCTAAAGTCGTGATGTTTGTTTT-3' \\
\hline GAPC-F & 5'-TCAGACTCGAGAAAGCTGCTAC-3' \\
\hline GAPC-R & 5'-GATCAAGTCGACCACACGG-3' \\
\hline
\end{tabular}




\subsection{Results}

4.3.1 Analysis of epicuticular wax crystals and wax composition of cer9-1 mutants

\subsubsection{Altered epicuticular wax crystal formation of cer9-1}

Scanning electron microscopy was performed to analyze the epicuticular crystals on cer9-1 mutants. The surface imaging of cer9-1 performed by Koorneef et al. (1989) was of relatively low resolution. Compared with the corresponding wild-type Ler-WT ecotype, the total wax crystal density of cer9-1 was significantly decreased (Figure 21A, D). The typical tube-shape wax crystals found on Ler-WT stems were missing on cer9-1 stems (Figure 21C, F). Instead, the major wax crystal shapes on cer9-1 stem were flat irregular shaped protrusions. These wax crystal phenotypes were consistent with and expand upon the previous analysis by SEM of cer9-1 stem surfaces (Koornneef et al., 1989). 


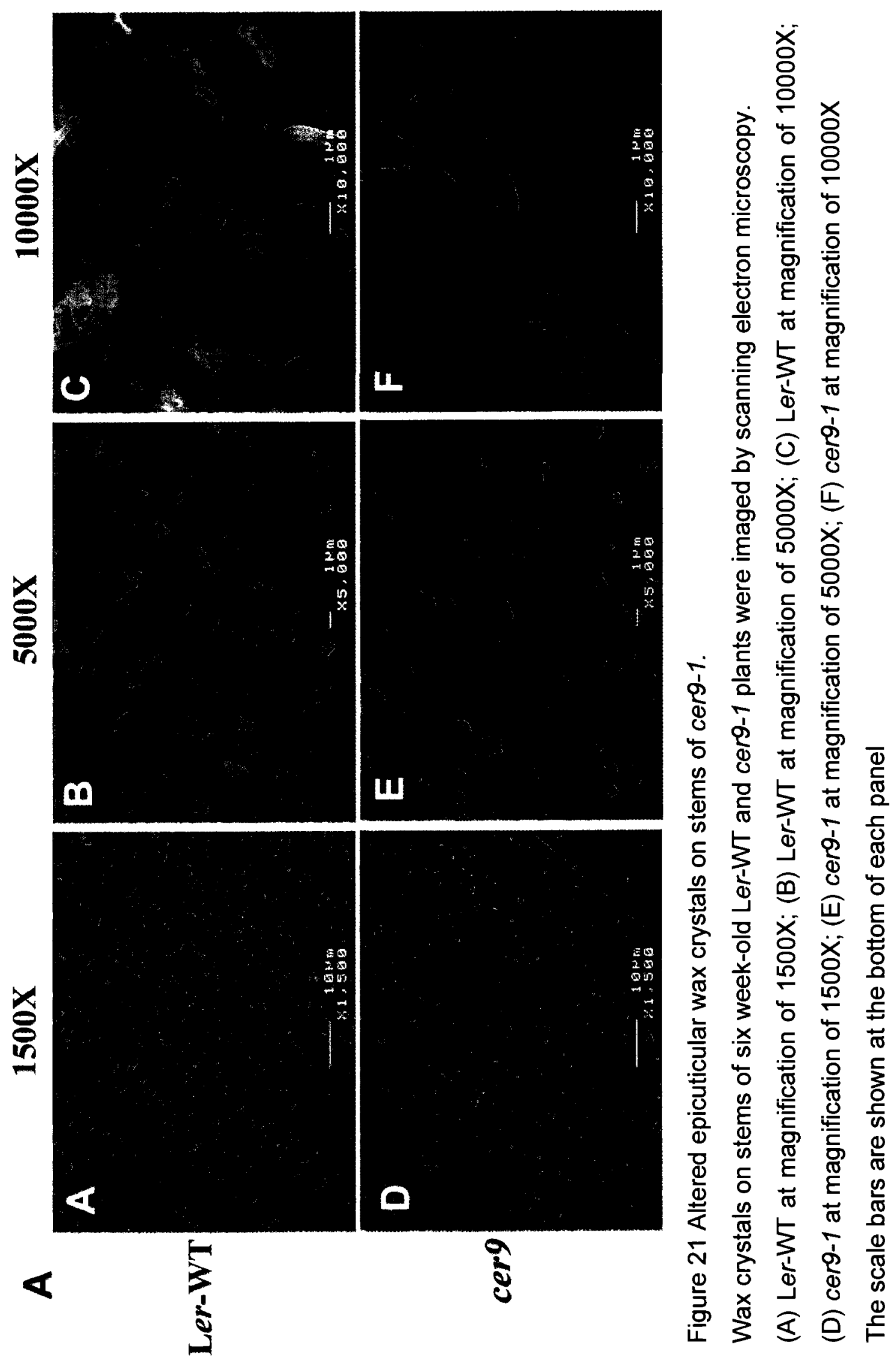




\subsubsection{Cuticular Wax Composition on stems and leaves of cer9-1}

Gas chromatography was performed to analyze the total cuticular wax load and composition of cerg-1 stems and leaves, compared to the corresponding wild-type ecotype (Ler-WT). The major wax components on Ler-WT leaves and stems are different (Jenks et al., 1995). C29 alkane, C29 secondary alcohol, and C29 ketone are the major wax components of Arabidopsis wild-type stems, while leaves have a much higher proportion of C31 alkane than C29 alkane and there are no detectable secondary alcohols and ketones (Table 7; Jenks et al., 1995). C24-C26 free fatty acids and primary alcohols are present on both stems and leaves (Figure 22; Jenks et al., 1995). The total wax load on cer9-1 stems was decreased to $62 \%$ of wild type (Ler) levels, while the total wax load on cer9-1 leaves was similar to the load on Ler-WT leaves (Table 7). On cer91 stems, C24:0 and C26:0 free fatty acids were significantly increased, while C28:0 and C30:0 free fatty acids were highly decreased compared to wild type (Figure 22A). Primary alcohols of chain lengths $C 24, C 26$, and $C 28$ were increased on cer9-1 stems, whereas the C30 primary alcohol was decreased to $42 \%$ of wild-type levels (Figure 22A). With regards to alkane-forming pathway products, all components were decreased by about $75 \%$, except for $\mathrm{C} 26 / \mathrm{C} 28$ aldehydes, whereas $\mathrm{C} 27$ alkanes were not significantly affected (Figure 22C). On cer9-1 leaves, C24:0 and C26:0 free fatty acids were increased 57- and 31- fold, respectively, relative to that on wild-type leaves, while $\mathrm{C} 28: 0$ and $\mathrm{C} 30: 0$ free fatty acids were decreased to nearly undetectable levels. Primary alcohols of chain length C24, C26, and C28 were decreased on cer9-1 leaf wax, while C30 primary alcohol was unaffected (Figure 22B). On leaves, all wax components 
of all chain lengths produced in the alkane-forming pathway were dramatically decreased (Figure 22D). 
Table 7 Chemical composition of stem and leaf cuticular wax of Ler-WT and cer9-1

\begin{tabular}{|l|l|l|l|l|}
\hline & Ler-WT Stems & cerg-1 Stems & Ler-WT Leaves & cer9-1 Leaves \\
\hline Total Wax Load & $17.79 \pm 1.12$ & $11.06 \pm 1.61$ & $2.28 \pm 0.15$ & $2.29 \pm 0.51$ \\
\hline C24 Free Fatty Acid & $0.04 \pm 0.003$ & $0.29 \pm 0.05$ & $0.006 \pm 0.002$ & $0.35 \pm 0.03$ \\
\hline C26 Free Fatty Acid & $0.02 \pm 0.001$ & $0.28 \pm 0.03$ & $0.01 \pm 0.006$ & $0.31 \pm 0.03$ \\
\hline C28 Free Fatty Acid & $0.07 \pm 0.002$ & $0.04 \pm 0.005$ & $0.007 \pm 0.003$ & $0 \pm 0$ \\
\hline C30 Free Fatty Acid & $0.34 \pm 0.02$ & $0.12 \pm 0.01$ & $0.039 \pm 0.01$ & $0.004 \pm 0.001$ \\
\hline C24 Primary Alcohol & $0.05 \pm 0.003$ & $0.06 \pm 0.006$ & $0.01 \pm 0.005$ & $0.001 \pm 0.002$ \\
\hline C26 Primary Alcohol & $0.28 \pm 0.01$ & $0.99 \pm 0.07$ & $0.014 \pm 0.005$ & $0.001 \pm 0.0008$ \\
\hline C28 Primary Alcohol & $0.55 \pm 0.01$ & $1.31 \pm 0.01$ & $0.017 \pm 0.004$ & $0.004 \pm 0.0008$ \\
\hline C30 Primary Alcohol & $0.31 \pm 0.003$ & $0.13 \pm 0.01$ & $0.029 \pm 0.009$ & $0.03 \pm 0.003$ \\
\hline C26 Aldehyde & $0.02 \pm 0.002$ & $0.05 \pm 0.01$ & $0.001 \pm 0.0002$ & $0.001 \pm 0.0001$ \\
\hline C28 Aldehyde & $0.11 \pm 0.01$ & $0.12 \pm 0.04$ & $0.009 \pm 0.004$ & $0.001 \pm 0.0008$ \\
\hline C30 Aldehyde & $0.61 \pm 0.07$ & $0.21 \pm 0.03$ & $0.018 \pm 0.007$ & $0.01 \pm 0.003$ \\
\hline C27 Alkane & $0.06 \pm 0.004$ & $0.08 \pm 0.007$ & $0.006 \pm 0.002$ & $0.002 \pm 0.001$ \\
\hline C29 Alkane & $6.63 \pm 0.41$ & $1.89 \pm 0.12$ & $0.057 \pm 0.01$ & $0.01 \pm 0.006$ \\
\hline C31 Alkane & $0.18 \pm 0.007$ & $0.04 \pm 0.009$ & $0.05 \pm 0.01$ & $0.006 \pm 0.002$ \\
\hline C33 Alkane & $0.0075 \pm 0.003$ & $0.03 \pm 0.002$ & $0.13 \pm 0.001$ & $0.03 \pm 0.007$ \\
\hline C29 Secondary Alcohol & $4.17 \pm 0.23$ & $1.51 \pm 0.11$ & $0.005 \pm 0.001$ & $0.001 \pm 0.0009$ \\
\hline C31 Secondary Alcohol & $0.08 \pm 0.009$ & $0.03 \pm 0.004$ & $0.01 \pm 0.004$ & $0.007 \pm 0.004$ \\
\hline C29 Ketone & $1.73 \pm 0.11$ & $0.45 \pm 0.02$ & $0 \pm 0$ & $0 \pm 0$ \\
\hline
\end{tabular}

Unit: $\mu \mathrm{g} / \mathrm{cm}^{2}$. Each value is the average of five replicates $(n=5)$, and \pm standard deviation is shown. 
Figure 22 Stem and leaf cuticular wax compositions of cer9-1 and the corresponding wild type ecotype (Ler-WT).

(A) Primary alcohols and free fatty acids components of cer9-1 stem wax sorted by chemical class and chain length.

(B) Primary alcohols and free fatty acids components of cerg-1 leaf wax sorted by chemical class and chain length.

(C) Alkane forming pathway components of cer9-1 stem wax sorted by chemical class and chain length.

(D) Alkane forming pathway components of cerg-1 leaf wax sorted by chemical class and chain length.

All values are mean, presented in $\mu \mathrm{g} / \mathrm{cm}^{2}$, derived from five replicates $(n=5)$. Error bars represent the standard deviation. 

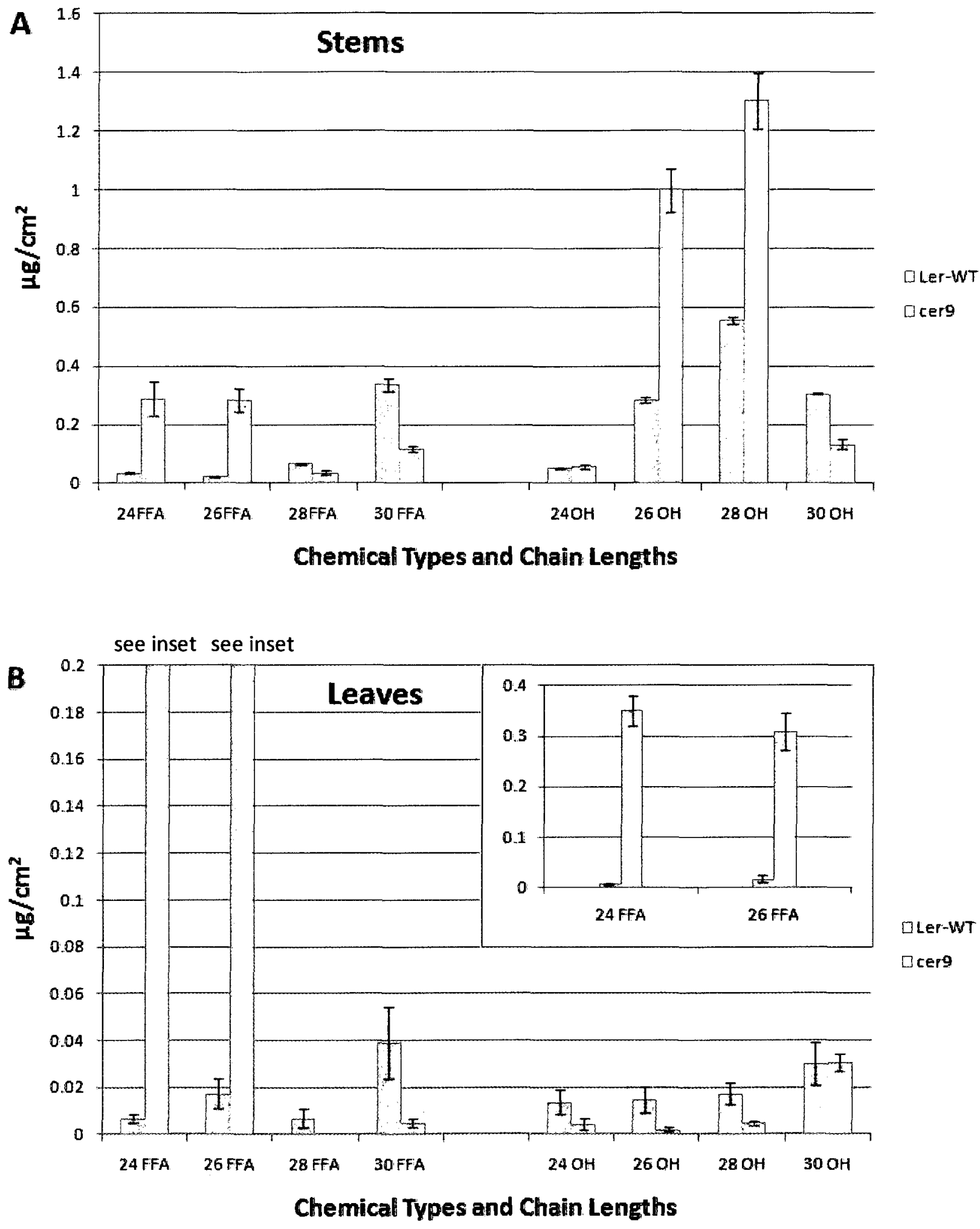

FFA: Free fatty acids; $\mathrm{OH}$ : Primary alcohols 

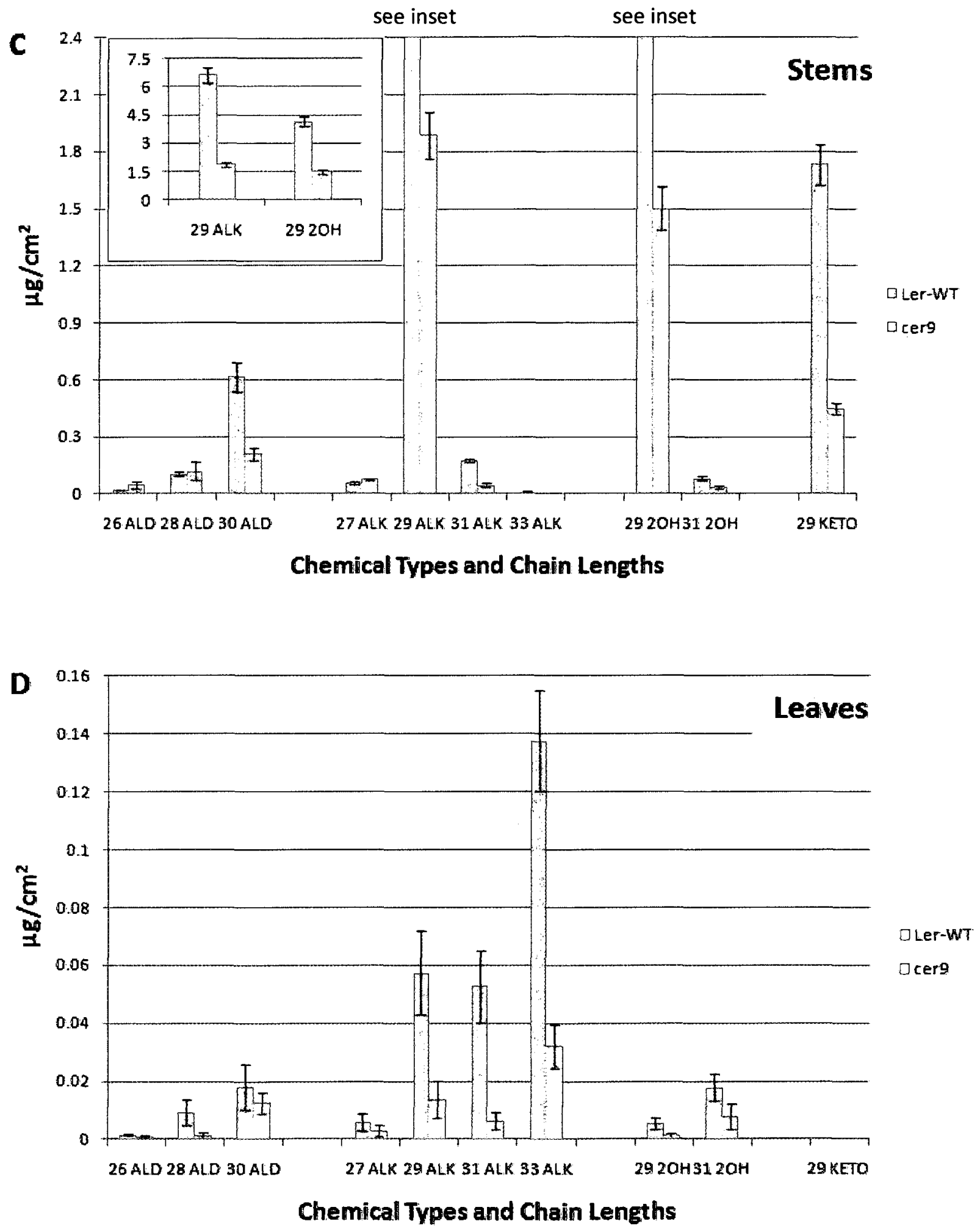

ALK: Alkanes; ALD: Aldehydes; 2OH: Secondary alcohols; KETO: Ketones 


\subsubsection{Cuticular permeability of cer9-1}

A TB assay was performed in young seedlings to test for defects in cer9-1 cuticle permeability. The leaves of wild-type seedlings did not show TB staining, as expected for plants with a complete cuticle (Figure 23A). The leaves of cer9-1 seedlings showed staining in the distal portions of the leaves (Figure 23B), indicative of a cuticle permeability defect of the class II type according to the classification nomenclature of Tanaka et al (2003).

Measurements of non-stomatal water loss from plant tissues to the environment were performed. Rosette leaves and stems showed no difference in the rate of water loss between cer9-1 and Ler-WT (Figure 24A, B). According to T-test statistical analysis, with $\mathrm{df}=6, \mathrm{t}$ must be at least 4.3 to reach $\mathrm{p}$-value $<0.05$. Our result was $t=0.3503$, so by conventional criteria, this difference is considered to be not statistically significant.

As showed in Figure 25, chlorophyll leached much faster from the detached rosette leaves of cer9-1 plants in $80 \%$ ethanol compared to leaves of wild-type plants. After 40 minutes of being immersed in $80 \%$ ethanol, the concentration of chlorophyll leached from cer9-1 rosette leaves was almost 12 times greater than that from Ler-WT rosette leaves. At two hours following immersion in $80 \%$ ethanol, $8.3 \pm 0.3 \mu \mathrm{mol} / \mathrm{g}$ of chlorophyll had leached from cer9-1 rosette leaves, while only $1.3 \pm 0.2 \mu \mathrm{mol} / \mathrm{g}$ of chlorophll had leached from Ler-WT rosette leaves. 


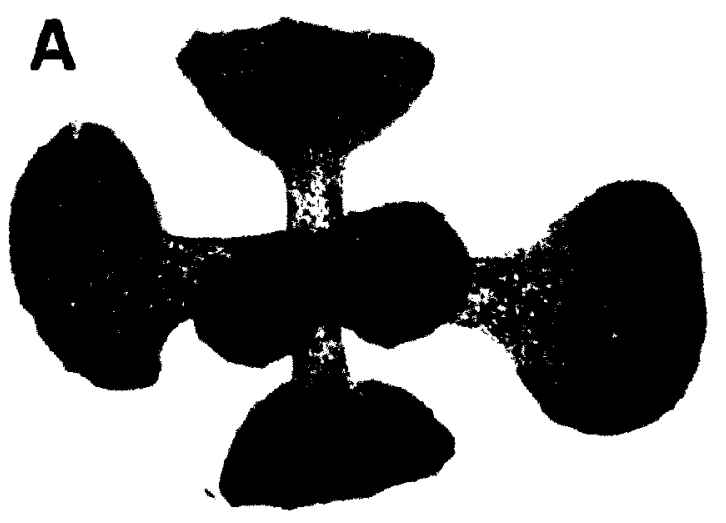

\section{Ler-WT}

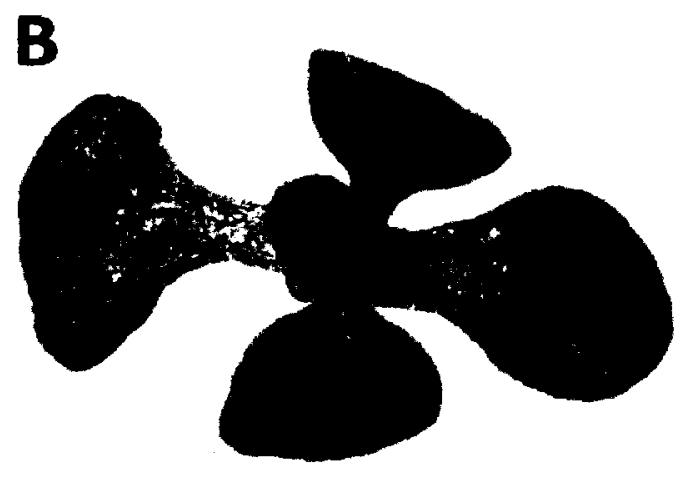

cerg

Figure 23 Toluidine blue staining on leaves of Ler-WT and cer9-1 seedlings

The TB staining assay was conducted on two week old seedlings of (A) Ler-WT, and (B) cer9-1. 
Figure 24 Cuticular transpiration of Ler-WT and cer9-1

(A) Fresh weight assay with leaves of 5-week old cer9-1 and Ler-WT plants, where the rate of water loss per gram of fresh weight tissue from rosette leaves was measured at the indicated time points $(n=5 ; \pm$ standard deviation).

(B) Fresh weight assay with stems of 5-week old cer9-1 and Ler-WT plants, where the rate of water loss per gram of fresh weight tissue from stems was measured at the indicated time points $(n=5 ; \pm$ standard deviation). 

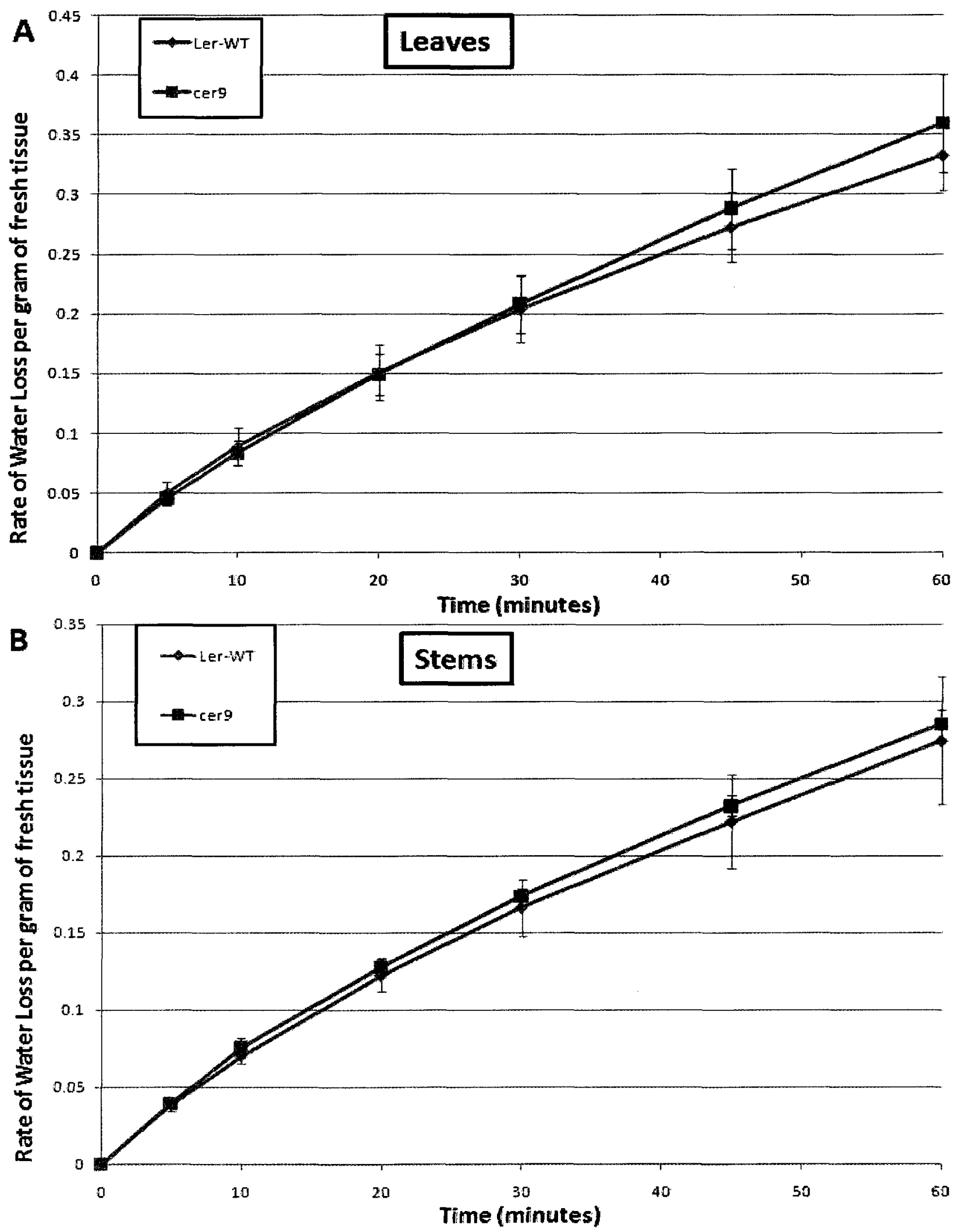


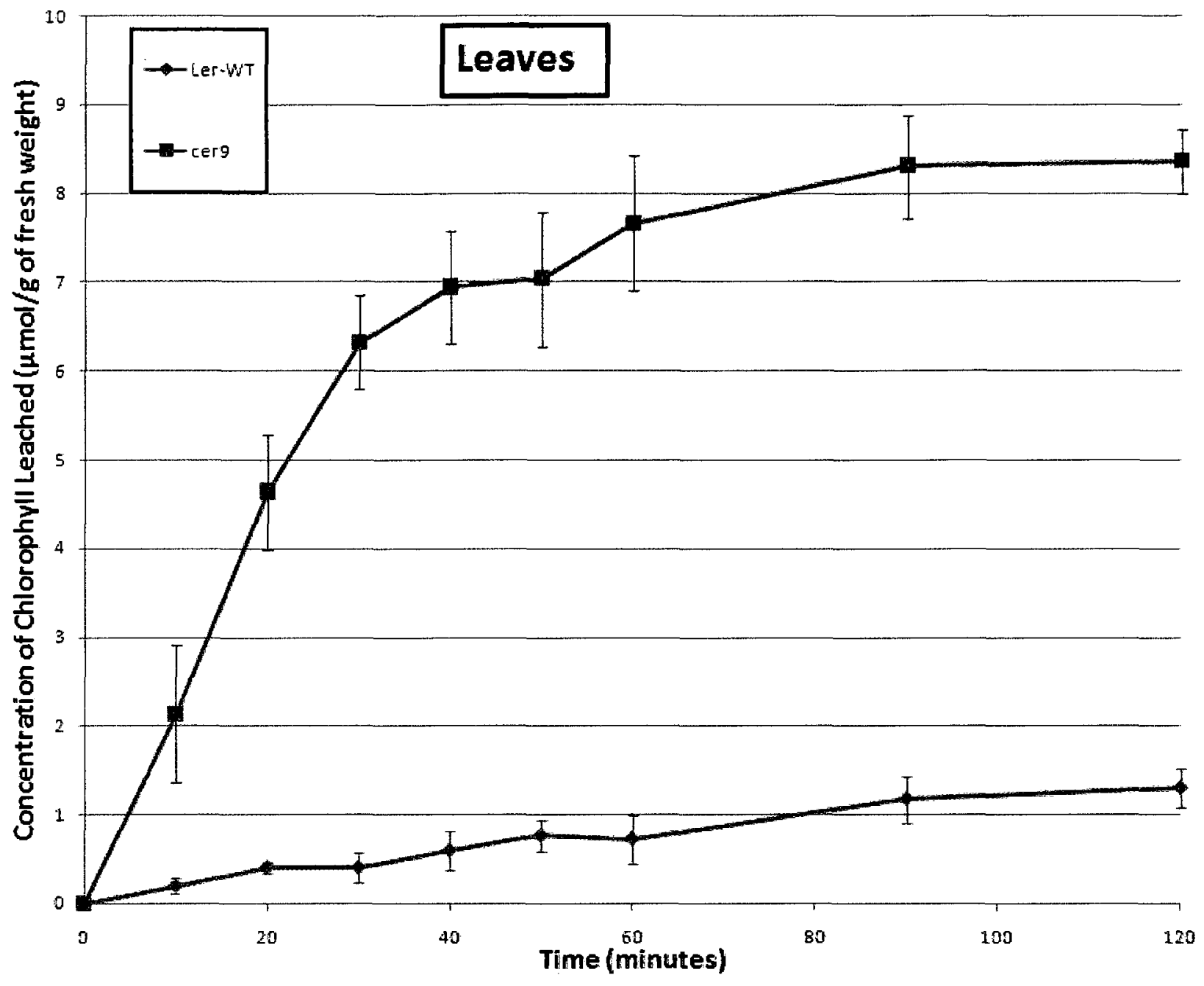

Figure 25 Rate of chlorophll leaching in cer9-1 and Ler-WT leaves

The concentration of chlorophyll leached over 2 hours into solution from 3 weekold rosette leaves of cer9-1 and Ler-WT immersed in $80 \%$ ethanol $(n=10 ; \pm$ standard deviation). 


\subsubsection{Positional mapping of cer9-1}

As described above, the alterations in cuticular wax on stems and leaves of cer9-1 are very dramatic. Identification of the CER9 gene may give important insights into biosynthesis, regulation and/or transport of cuticular waxes. We therefore initiated a map-based cloning approach to identify CER9.

Genetic analysis confirmed that the glossy phenotype of cer9-1 is recessive by observing F1 plants derived from a cross between Ler-WT and cer9-1 mutants (data not shown). The F1 plants were glaucous. Furthermore, 200 segregating F2 plants showed 152 wild-type and 48 glossy plants. A chi-squared statistical analysis was used to determine if the segregation data fits the hypothesis that cer9-1 is a recessive mutation. $\chi^{2}$ was 0.11 and therefore lies confidently within the range that is to be expected for $95 \%$ of sample sets occurring from segregation of a single recessive gene. So we can accept that cer9-1 segregates at a $3: 1$ ratio. Therefore, it is a recessive mutation.

To determine the position of the CER9 loci, an F2 mapping population was constructed containing 1046 glossy individuals derived from a cross of cer9-1 with Col-0. By analyzing 40 glossy plants from this population, the mutation causing the glossy phenotype was found to be linked to the simple sequence length polymorphism (SSLP) marker nga1107, which is at position $18.09 \mathrm{Mbp}$ on the bottom of chromosome IV (Figure 26A, B). This result was consistent with previous genetic linkage analysis that indicated CER9 was located at the bottom of chromosome IV (Koornneef et al., 1989). For fine mapping, new insertion-deletion (InDel) markers were developed near to this mapping region. CER9 gene was mapped to a region flanked by markers CER452834 and CER460758, which are located at positions $12.85 \mathrm{Mbp}$ and $18.51 \mathrm{Mbp}$, respectively 
(Figure 26C). This gave a recombination frequency of $7 \%$ and $14 \%$, respectively, based on 100 samples each. To further narrow down the locus, more InDel markers were designed between the flanking markers (Table 8) and the entire mapping population of 1046 individuals was used to identify recombinants. The CER9 locus mapped to a region between marker CER458597 and marker CER460644, which are located between $16.21 \mathrm{Mbp}$ and $16.53 \mathrm{Mbp}$, respectively (Figure 26D). Five bacterial artificial chromosomes (BACs) covered that region: T16L1, F17I5, F28A23, F10M10, and T4L20 (Figure 26E).

During the course of this mapping procedure, it came to our attention that a collaborator, the lab of Professor Matthew Jenks (Purdue University, West Lafayette, Indiana, USA), had also established a mapping population for CER9. We needed additional recombinants to further narrow down the region containing CER9. Therefore, we shared out mapping information with the Jenks laboratory and they were able to further narrow down the region containing CER9 to $61 \mathrm{Kbp}$ using their larger mapping population. They sequenced the entire $61 \mathrm{Kbp}$ region in the cer $9-1$ mutant and found a single non-silent missense mutation (personal communication). However, further experimentation is needed to confirm the identification of CER9 and this is beyond the timeframe and scope of this M.Sc. thesis. 
Figure 26 Genetic mapping of CER9 the locus

(A and B) First-pass mapping of CER9. CER9 was found to be linked to SSLP marker nga1107. The recombination frequency (RF) was based on 40 samples. The physical position of the marker is indicated.

(C) Flanking markers of CER9 loci. CER9 was mapped to a genetic interval between markers CER452824 and CER460758. The recombination frequency (RF) of each marker was based on 100 samples and is indicated below the marker names.

(D) Fine genetic mapping of CER9. Analyzing 1046 F2 mapping population plants, CER9 was further narrowed down to a region between markers CER458597 and CER460644. Markers and the numbers of recombination events identified are indicated. (E) Five bacterial artificial chromosomes (BACs) covered the region between markers CER458597 and CER460644. Yellow lines represent five different BACs. Red lines in the BAC represent the position of each marker in the BAC. 


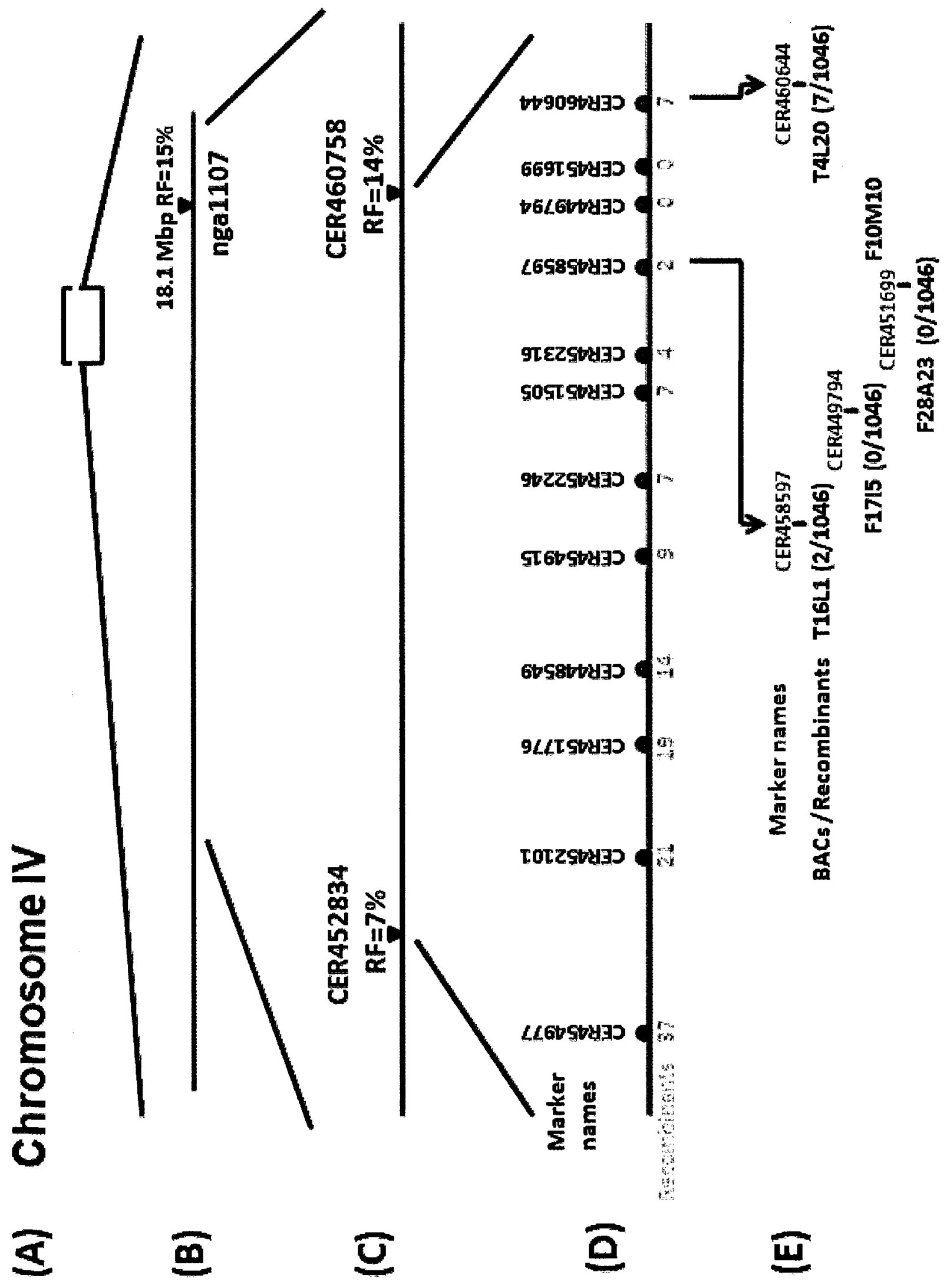




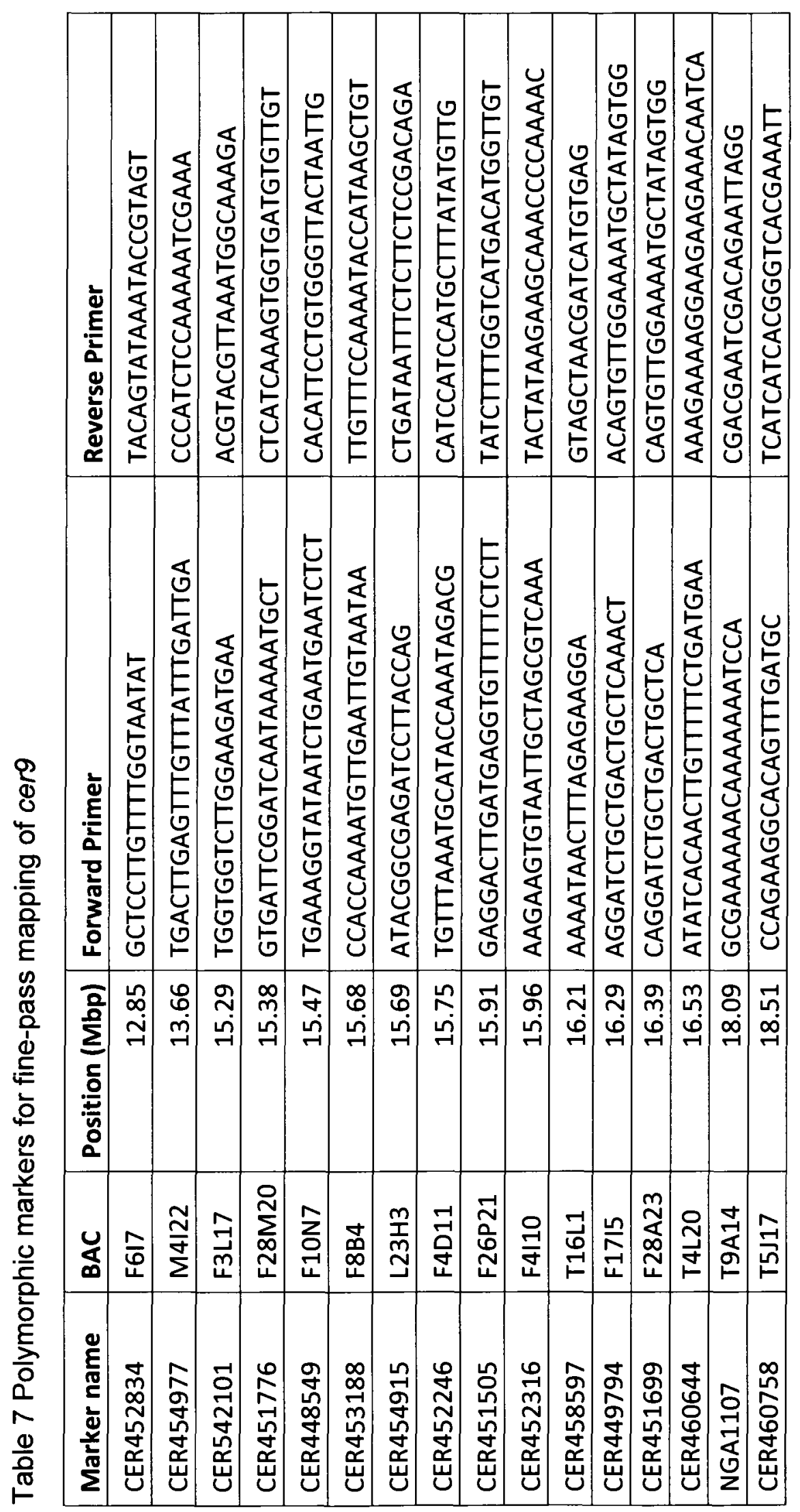


4.3.4 Gene expression patterns of wax and cutin-associated genes in cerg-1

The candidate CER9 gene described above is not predicted to be a metabolic enzyme, based on its complex wax-altered phenotype. It may be a regulator and therefore we decided to monitor the gene expression levels of cuticle-associated genes in cer9-1 to test if they were up or down regulated. RT-PCR was performed on the set of cuticle-associated genes and a constitutive control (GAPC) in both leaves and stems, comparing transcript levels in wild-type versus cer9-1. We analyzed the expression of KCS1 (Todd et al., 1999) and CER6 (Fiebig et al., 2000), which are involved in elongation of fatty acyl chains; ATT1 (Xiao et al., 2004) is a cytochrome P450 enzyme involved in biosynthesis of cutin; LACS2 (Schnurr et al., 2004) and CER8/LACS1 (Lu et al., 2009) are acyl-activating enzymes involved in both wax and cutin biosynthesis; CER2 (Negruk et al., 1996; Xia et al., 1996; St-Pierre et al., 1998) encodes a putative CoA-dependent acyltransferase involved in cuticle biosynthesis; CER4 (Rowland et al., 2006) is an alcohol-forming fatty COA reductase involved in production of primary alcohols in the cuticle; CER5 (Pighin et al., 2004) encodes an ABC transporter required for wax transport to the cuticle; CER1 (Aarts et al., 1995) and CER3 (Chen et al., 2003; Rowland et al., 2007) are postulated to be integral membrane enzymes that contain a di-iron active site involved in biosynthesis of wax, and GAPC is the constitutively expressed control. However, none of these genes showed detectable changes in cer9-1 compared to Ler-WT (Figure 27). 


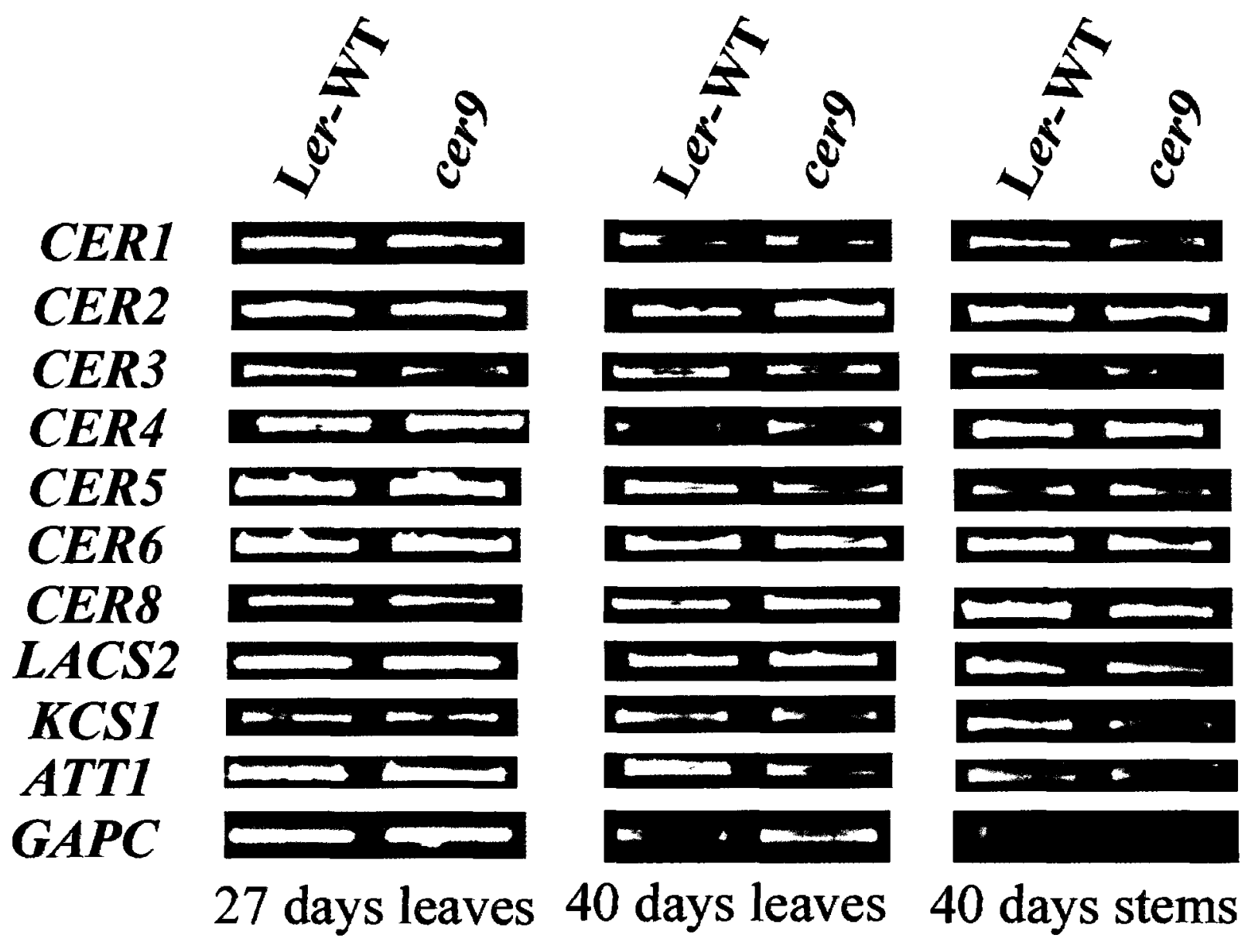

Figure 27 RT-PCR analysis of transcripts encoding proteins involved in wax and cutin biosynthesis in Ler-WT and cer9-1

Each panel is the amplification product generated by RT-PCR and is representative of the transcript levels of the genes indicated on the left. RNA for RT-PCR was extracted from 27 day-old rosette leaves of Ler-WT and cer9 (column 1), and 40 days old rosette leaves and stems of Ler-WT and cer9 (column 2 and 3, respectively). The last row is the RT-PCR product of GAPC, which is constitutively expressed, and thus is a loading control for the corresponding lanes in the panels above. 


\subsection{Discussion}

The cer9-1 mutant has a dramatic and very unique alteration in its cuticular wax composition (Jenks et al., 1995; and results shown here). In wild-type Arabidopsis plants, the major leaf wax constituents are C29-C33 alkanes, whereas in cer9-1 these constituents are nearly absent, and instead C24 and C26 free fatty acids predominate almost to the exclusion of all other waxes. In stem wax, the C24 and C26 free fatty acids are also elevated in cer9-1. However, in contrast to the leaf phenotype, stem wax also has higher levels of C26 and C28 primary alcohols but lower levels of C30 primary alcohols relative to wild-type. Wax esters of cer9-1 stems are increased relative to wildtype, but the chain length distribution has not been reported. Alkanes, secondary alcohols and ketones of chain lengths $\mathrm{C} 29$ and higher are substantially reduced. Thus, both chemical class and chain lengths of wax constituents are altered in cer9-1, and there are substantial differences in the effects between the alterations exhibited in stem and leaf wax. Jenks et al. (1995) speculated that the CER9 gene product is involved in chain-length specific transfer of fatty acyl-chains through the wax biosynthetic pathway, but that the chain length affected is different depending on the tissue type. Proteins involved in such a transfer process have not been identified, nor is there evidence that such a transfer process needs to be facilitated. It is also possible that CER9 is a regulator of wax biosynthesis and that the complex alterations in wax accumulation observed in cer9-1 leaves and stems may be due to misregulation of multiple genes and/or proteins involved in wax biosynthesis.

We also investigated whether cer9-1 has a defect in the permeability of its cuticle using three different assessments of cuticular permeability. Although there was no major effect on cuticular transpiration (water loss assay) and a relatively minor effect on 
staining by toluidine blue, cer9-1 did exhibit a large difference from wild-type in the rate of chlorophyll leaching. Differences in cuticle permeability at different developmental stages may account in part for these conflicting results. The rosette leaves used in the chlorophyll-leaching assay were taken from 3-week old plants, which still have a developing cuticle, whereas the leaves used in the fresh water loss assay were taken from 5 week-old plants. The water loss assay should be repeated on younger leaves to determine if the cuticle is more dramatically affected, in terms of permeability, in younger plants. The general integrity of the cuticle structure can also be investigated at various developmental stages using transmission electron microscopy (TEM), but this is a relatively crude measurement of cuticle structure. Also, it will be important to determine if there are alterations in cutin composition in cer9-1, which can also affect the ultrastructure of the cuticle and thus the passage of chlorophyll through the cuticular membrane. For example, lacs $1 /$ cer 8 and lacs 2 mutants affect both cutin and wax composition (Lu et al., 2009) and the same may be true in the cer9-1 mutant.

Important progress has been made towards the cloning of CER9 by my localization of the interval containing CER9 to an approximately $320 \mathrm{Kbp}$ region on chromosome IV. As mentioned above in the Results section, the interval containing CER9 was further localized to $61 \mathrm{Kbp}$ in collaboration with the laboratory of Matthew Jenks and a candidate gene for CER9 has been identified through sequencing of this region. Future work is needed to verify the identity of the CER9 gene, which is work in progress between the two labs. Ultimately, detailed characterization of the CER9 gene and gene product will hopefully reveal the precise role of CER9 in cuticular wax metabolism. 
Towards this end, I tested the possibility that the cerg-1 mutant was affecting the expression of other genes involved in the biosynthesis and/or secretion of wax and cutin components. This analysis did not require knowing the identity of the CER9 gene. As described above, the complicated phenotype of cer9-1 suggests that CER9 may be regulator of cuticle development rather than a biosynthetic enzyme. Also, the candidate gene for CER9 and genes in the vicinity of the CER9 loci are not predicted to encode enzymes involved in cutin or wax metabolism. We tested 10 genes involved in wax or cutin production, but the transcript levels of these genes were not altered in the cer9-1 mutant. However, we did not exhaustively evaluate all genes involved in cuticle development. These other genes could be tested in the future or a DNA microarray may reveal alterations of gene expression on a genome-wide scale. Alternatively, the effects of cer9-1 could be exerted at the protein level, with changes in the levels or activities of proteins involved in wax and/or cutin metabolism. This is more complicated to evaluate than changes at the transcriptional level. There are also a myriad of indirect effects on cuticle development that could be taking place in the cer9-1 mutant, which would cause the dramatic alterations in wax composition in leaves and stems. Again, identification of CER9 is critical for evaluating this possibility further. 


\section{Chapter 5 General Conclusion and Discussion}

Mutants of Arabidopsis that have wax deficiencies are often easily identified by their glossy stems. Detailed analyses of the wax composition defects in these mutants, which are typically called eceriferum (cer), have provided enormous insights into the nature of wax biosynthetic pathways. Furthermore, the cloning of CER genes has allowed the characterization of key wax biosynthetic enzymes and transporters (Samuels et al., 2008). However, not all of the known CER loci have been cloned and screens for cer mutants have never been done to saturation. Therefore, much remains to be elucidated in understanding cuticular wax biosynthesis and its regulation. To this end, I examined a set of previously uncharacterized Arabidopsis mutants affected in cuticular wax production.

In Chapter 2, the goal was to phenotypically group the new wax deficient mutants, termed line mutants, which were previously identified by the Rowland lab, and then use this information to guide complementation crosses to determine which line mutants were alleles of existing cer mutants and which were potentially mutants of novel cer loci. The phenotypes analyzed were wax crystal density and shape by scanning electron microscopy and wax composition by gas chromatography. This approach was successful, although in hindsight complementation crosses, without detailed phenotypic characterization of these line mutants, may have been just as quick. The Rowland lab possesses over 50 additional line mutants that have not yet been characterized, but complementation crosses between all these mutants and the nearly 30 known cer mutants would be cumbersome. Therefore, my approach may be useful in analyzing 
this larger set. The key is to generate robust data on the wax compositions by gas chromatography and have an effective means to compare the profiles.

In Chapter 3, I began further characterization of what were thought to be mutants of two novel CER loci. More detailed phenotypic characterization, positional mapping, and complementation crosses revealed, however, that they were instead new alleles of cer6 and cer19, which we termed cer6-3 and cer19-2, respectively. Cer6-3 is a weak allele, which is what caused me to initially think it was not a mutation in CER6. Sequencing of the CER6 gene in this mutant background revealed that amino acid 317 of the encoded $\beta$-ketoacyl-CoA synthase is important for enzymatic activity, and that changing the residue from a small hydrophobic residue (alanine) to a larger hydrophobic residue (valine) does not completely abolish activity. The importance of this amino acid for activity of this class of enzyme has not been tested before, although it is a conserved residue. Identification of the new cer19-2 allele will be important for the subsequent cloning and characterization of CER19. This follow-up work is being carried out in collaboration between the Rowland lab and the laboratory of Professor Ljerka Kunst at the University of British Columbia. The presence of independent mutations in cer19-1 and cer19-2 that reside in the same candidate gene will be good evidence for CER19 identification. Also, phenotypic characterization is typically carried out in two independent allelic lines to eliminate the possibility of linked background mutations modifying the phenotype. Careful examination of the wax composition defect in cer19-1 and cer19-2 indicated that CER19 is likely involved in elongation of C28 acyl chains to C30 acyl chains. This is a key step in wax biosynthesis leading to production of C30 and 
longer acyl-CoAs, which are the major substrates for modification in the alkane-forming pathway.

In Chapter 4, I initiated a map-based cloning approach to identify the CER9 gene. The cuticular wax phenotype of cer9-1 is intriguing, with alterations in every major chemical class and chain length. In cer9-1 leaves, the major wax components are C24 and $\mathrm{C} 26$ free fatty acids rather than alkanes and primary alcohols. This caused an alteration in the permeability of the leaf cuticle, which had not been reported before. Future work needs to be done to determine if leaf cutin is also affected in cer9-1. Interestingly, there are tissue-specific wax alterations in cer9-1. In cer9-1 stems, C26 and C28 primary alcohols are not reduced as in leaves, but are instead significantly increased. It is possible that regulation of wax metabolism is different in leaves and stems of Arabidopsis. Indeed, CER9 itself may encode a regulator. If so, it does not seem to act at the transcriptional level, at least for the 10 genes tested here, which are known to be important for wax or cutin metabolism. Using linkage to molecular markers on chromosome IV, I narrowed down the region of genomic DNA containing CER9 to a $320 \mathrm{kbp}$ interval. I could not narrow down the region further because there were no more useful recombinants in my mapping population. A larger mapping population was needed. The laboratory of Professor Matthew Jenks at Purdue University also had a mapping population for cer9, and so we shared our mapping information and they were able to further narrow the segment containing CER9 using their larger mapping population. A candidate gene has been identified, but further molecular characterization is required to determine if this gene is indeed CER9. Molecular complementation of the cer9-1 mutant by transformation of the wild-type gene is being carried out. Additional 
alleles have not been identified in this gene (e.g. T-DNA inserts) and silencing of the gene using RNA interference is also being carried out. The subsequent characterization of CER9 will continue in collaboration between the Rowland and Jenks laboratories.

In closing, this thesis has made important progress in the phenotypic characterization of Arabidopsis cer9 and cer19 mutants affected in cuticular wax metabolism. The future cloning and characterization of CER9 and CER19 is anticipated to provide valuable insights into the molecular mechanisms governing cuticular wax production. 


\section{References}

1. Aarts MGM, Keijzer CJ, Stiekema WJ and Pereira A. (1995). Molecular characterization of the CER1 gene of Arabidopsis involved in epicuticular wax biosynthesis and pollen fertility. Plant Cell 7: 2115-2127.

2. Aharoni A, Dixit S, Jetter R, Thoenes E, van Arkel G and Pereira A. (2004). The SHINE clade of AP2 domain transcription factors activates wax biosynthesis, alters cuticle properties, and confers drought tolerance when overexpressed in Arabidopsis. Plant Cell 16: 2463-2480.

3. Alonso JM and Ecker JR. (2006). Moving forward in reverse: genetic technologies to enable genome-wide phenomic screens in Arabidopsis. Nat Rev. Genet. 7: 524-36.

4. Ariizumi T, Hatakeyama K, Hinata K, Sato S, Kato T, Tabata S and Toriyama K. (2003). A novel male-sterile mutant of Arabidopsis thaliana, faceless pollen-1, produces pollen with a smooth surface and an acetolysis-sensitive exine. Plant Mol. Biol. 53: 107-116.

5. Arondel V, Vergnolle C, Cantrel C and Kader J-C. (2000). Lipid transfer proteins are encoded by a small multigene family in Arabidopsis thaliana. Plant Science 157: 112.

6. Baker E. (1974). The influence of environment on leaf wax development in Brassica oleracea var. gemmifera. The New Phytologist 73: 955-966.

7. Baker E. (1974). The influence of environment on leaf wax development in Brassica oleracea var. gemmifera. New Phytol. 53: 107-16.

8. Baker EA and Hunt GM. (1979). Secondary alcohols from Fragaria leaf waxes. Phytochemistry 18: 1059-1060. 
9. Beaudoin F, Wu X, Li F, Haslam RP, Markham JE, Zheng H, Napier JA and Kunst L. (2009). Functional characterization of the Arabidopsis thaliana \{beta\}-ketoacyl-CoA reductase candidates of the fatty acid elongase. Plant Physiol. 150: 1174-1191.

10. Bessire $M$, Chassot $C$, Jacquat $A C$, Humphry M, Borel S, Petétot JM, Métraux JP and Nawrath C. (2007). A permeable cuticle in Arabidopsis leads to a strong resistance to Botrytis cinerea. The EMBO Journal 26: 2158-2168.

11. Bird D, Beisson F, Brigham A, Shin J, Greer S, Jetter R, Kunst L, Wu X, Yephremov A and Samuels L. (2007). Characterization of Arabidopsis ABCG11MBC11, an ATP binding cassette $(A B C)$ transporter that is required for cuticular lipid secretion. The Plant Journal 52: 485-498.

12. Blacklock BJ and Jaworski JG. (2006). Substrate specificity of Arabidopsis 3ketoacyl-CoA synthases. Biochem. Biophys. Res. Commun. 346: 583-90.

13. Bonaventure G, Salas JJ, Pollard MR and Ohlrogge JB. (2003). Disruption of the FATB gene in the Arabidopsis demonstrates an essential role of saturated fatty acids in the plant growth. The Plant Cell 15: 1020-1033.

14. Bonaventure G, Beisson F, Ohlrogge $J$ and Pollard M. (2004). Analysis of the aliphatic monomer composition of polyesters associated with Arabidopsis epidermis: occurrence of octadeca-cis-6, cis-9-diene-1,18-dioate as the major component. Plant J. 40: $920-30$.

15. Bortiri E, Jackson D and Hake A. (2006). Advances in maize genomics: the emergence of positional cloning. Curr. Opin. Plant Biol. 9: 164-71

16. Broun P, Poindexter P, Osborne E, Jiang C-Z and Riechmann JL. (2004). WIN1, a transcriptional activator of epidermal wax accumulation in Arabidopsis. Proc. Nat. 
Acad. Sci. USA 101: 4706-4711.

17. Caldicott $A B$ and Eglington G. (1976). Cutin acids from bryophytes: an $\omega-1$ hydroxy alkanoic acid in two liverwort species. Phytochemistry 15: 1139-43.

18. Cheesbrough T and Kolattukudy PE. (1984). Alkane biosynthesis by decarbonylation of aldehydes catalyzed by a particulate preparation from Pisum sativum. Proc. Nat. Acad. Sci. USA 81: 6613-6617.

19. Chen X, Goodwin SM, Boroff VL, Liu X and Jenks MA. (2003). Cloning and characterization of the WAX2 gene of Arabidopsis involved in cuticle membrane and wax production. Plant Cell 15: 1170-1185.

20. Clough R, Matthis AL, Barnum SR and Jaworski JG. (1992). Purification and characterization of 3-ketoacyl-acyl carrier protein synthase from spinach: a condensing enzyme utilizing acetyl-CoA to initiate fatty acid synthesis. The Journal of Biological Chemistry 267: 20992-20998.

21. Cominelli E, Sala T, Calvi D, Gusmaroli G and Tonelli C. (2008). Over-expression of the Arabidopsis AtMYB41 gene alters cell expansion and leaf surface permeability. Plant J. 53: 53-64.

22. Deas JE, Aquilar FJ and Miller JH. (1974). Fine structure of the cuticle of female Onchocerca volvulus. J Parasitol. 60: 1006-12.

23. Debono A, Yeats TH, Rose JKC, Bird D, Jetter R, Kunst L and Samuel L. (2009). Arabidopsis LTPG is a glycosylphosphatidylinositol-anchored lipid transfer protein required for export of lipids to the plant surface. Plant Cell. 21: 1230-1238. 
24. Dunn TM, Lynch DV, Michaelson LV and Napier JA. (2004). A postgenomic approach to understanding sphingolipid metabolism in Arabidopsis thaliana. Ann. Bot. 93: 483-97.

25. Eigenbrode SD and Espelie KE. (1995). Effects of plant epicuticular lipids on insect herbivores. Annu Rev Entomol 40: 171-194.

26. Fehling $E$ and Mukherjee KD. (1991). Acyl-CoA elongase from a higher plant (Lunaria annua): metabolic intermediates of very-long-chain acyl-CoA products and substrate specificity. Biochimica et Biophysica Acta 1082: 239-46.

27. Fiebig A, Mayfield JA, Miley NL, Chau S, Fischer RL and Preuss D. (2000). Alterations in CER6, a gene identical to CUT1, differentially affect long-chain lipid content on the surface of pollen and stems. Plant Cell 12: 2001-2008.

28. Franke R, Briesen I, Wojciechowski T, Faust A, Yephremov A, Nawrath C and Schreiber L. (2005). Apoplastic polyesters in Arabidopsis surface tissues-a typical suberin and a particular cutin. Phytochemistry 66: 2643-2658.

29. Franke R, Hofer R, Briesen I, Emsermann M, Yephremov A and Schreiber L. (2009). The DAISY gene from Arabidopsis encodes a fatty acid elongase condensing enzyme involved in the biosynthesis of aliphatic suberin in roots and the chalazamicropyle region of seeds. Plant J. 57: 80-95.

30. Fulda M, Shockey J, Werver M and Wolter FP. (2002). Two long-chain acyl-CoA synthetases from Arabidopsis thaliana involved in peroxisomal fatty acid $\beta$-oxidation. Plant J. 32: 93-103.

31. Ghanevati M and Jaworski JG. (2000). Active-site residues of a plant membranebound fatty acid elongase $\beta$-ketoacyl-CoA synthase, FAE1 KCS. Biochimica et 
Biophysica Acta 1530: 77-85.

32. Graca J, Schreiber L, Rodrigues J and Pereira H. (2002). Glyceral and glyceryl esters of w-hydroxyacids in cutins. Phytochemistry 61:205-215.

33. Greer S, Wen M, Bird D, Wu X, Samuels L, Kunst L and Jetter R. (2007). The cytochrome P450 enzyme CYP96A15 is the mid-chain alkane hydroxylase responsible for formation of secondary alcohols and ketones in stem cuticular wax of Arabidopsis thaliana. Plant Physiology 145: 653-667.

34. Hannoufa A, McNevin J and Lemieux B. (1993). Epicuticular waxes of eceriferum mutants of Arabidopsis thaliana. Phytochemistry 33: 851-855.

35. Heredia A. (2003). Biophysical and biochemical characteristics of cutin, a plant barrier biopolymer. Biochim. Biophys. Acta 1620: 1-7.

36. Holloway PJ. (1969a). The effects of superficial wax on leaf wettability. Annals of Applied Biology 63: 145-153.

37. Holloway PJ. (1969b). Chemistry of leaf waxes in relation to wetting. Journal of the Science of Food and Agriculture 20: 124-128.

38. Holloway PJ. (1970). Surface factors affecting the wetting of leaves. Pesticide Science 1: 156-162.

39. Holloway PJ, Deas AHB and Kabarra AM. (1972). Composition of cutin from coffee leaves. Phytochemistry 11: 1443-47.

40. Holloway PJ, Brown GA, Baker EA and Macey MJK. (1977). Chemical composition and ultrastructure of the epicuticular wax in three lines of Brassica napus. Chem Phys Lipids. 19: 114-127.

41. Holloway PJ. (1982). Structure and histochemistry of plant cuticular membranes: an 
overview, in The Plant cuticle (eds D.F. Cutler, K.L. Alvin and C.E. Price), Academic Press, London, pp. 1-32.

42. Hooker TS, Millar AA and Kunst L. (2002). Significance of the expression of the CER6 condensing enzyme for cuticular wax production in Arabidopsis. Plant Physiology 129:1568-1580

43. Hooker TS, Lam P, Zheng $H$ and Kunst L. (2007). A core subunit of the RNAprocessing degrading exosome specifically influences cuticular wax biosynthesis in Arabidopsis. Plant Cell 19:904-913.

44. Hunneman DH and Eglington G. (1972). The constituent acids of gymnosperm cutins. Phytochemistry 11: 1989-2001.

45. Jander G, Norris SR, Rounsley SD, Bush DF, Levin IM and Last RL. (2002). Arabidopsis map based cloning in the post-genome era. Plant Physiol. 129: 440-50.

46. Jeffree C.E. (1996). Structure and ontogeny of plant cuticles, in Plant Cuticles: An Integrated Functional Approach (ed G. Kerstiens), BIOS Scientific Publishers, Oxoford, pp. 33-82.

47. Jeffree C.E. (2006). The fine structure of the plant cuticle, in Biology of The Plant Cuticle (eds M. Riederer and C. Müller), Blackwell Publishing, Oxford, pp. 11-125.

48. Jenks MA, Rich PJ and Ashworth EN. (1994). Involvement of cork cells in the secretion of epicuticular wax filaments on Sorghum bicolour (L.) Moench. International Journal of Plant Sciences 155: 506-518.

49. Jenks MA, Tuttle HA, Eigenbrode SD and Feldman KA. (1995). Leaf epicuticular waxes of the eceriferum mutants in Arabidopsis. Plant Physiology 108: 369-377. 
50. Joubes J, Raffaele S, Bourdenx B, Garcia C, Laroche-Traineau J, Moreau P, Domergue $F$ and Lessire R. (2008). The VLCFA elongase gene family in Arabidopsis thaliana: phylogenetic analysis, 3D modeling and expression profiling. Plant Mol Biol. 67: 547-66.

51. Kader J-C. (1996). Lipid-transfer proteins in plants. Annual Review of Plant Physiology and Plant Molecular Biology 47: 627-654.

52. Kannagara R, Branigan C, Liu $Y$ and Penfield T. (2007). The transcription factor WIN1/SHN1 regulates cutin biosynthesis in Arabidopsis thaliana. Plant Cell 19: 1278-94.

53. Kerstiens G. (1996). Signalling across the divide: a wider perspective of cuticular structure-function relationships. Trends in Plant Science 1: 125-129.

54. Khan AA and Kolattukudy PE. (1974). Decarboxylation of long chain fatty acids to alkanes by cell free preparations of pea leaves (Pisum sativum). Biochemical and Biophysical Research Communications 61: 1379-1386.

55. Kolattukudy PE. (1970). Reduction of fatty acids to alcohols by cell-free preparation of Euglena gracilis. Biochemistry 9: 1095-1102.

56. Kolattukudy PE. (1970). Biosynthesis of a lipid polymer, cutin: the structural component of plant cuticle. Biochem. Biophys. Res. Commun. 41: 299-305.

57. Kolattukudy PE. (1971). Enzymatic synthesis of fatty alcohols in Brassica oleracea. Archives of Biochemistry and Biophysics 142: 701-709

58. Kolattukudy PE, Buckner JS and Brown L. (1972). Direct evidence for a decarboxylation mechanism in the biosynthesis of alkanes in $B$. oleracea. Biochemical and Biophysical Research Communications 47: 1306-1313. 
59. Kolattukudy PE. (1974). Biosynthesis of a hydroxyl fatty acid polymer, cutin. Identification and biosynthesis of $16-0 x 0-9$ or 10 -hydroxy palmitic acid, a novel compound in Vicia faba. Biochemistry 13: 1354-63.

60. Kolattukudy PE, Rogers $L$ and Larson JD. (1981). Fatty acid synthase from the uropygial gland of goose. Methods in Enzymology 71103-109.

61. Kolattukudy P.E. (2001). Polyesters in higher plants. Advances in Biochemical Engineering/Biotechnology 71: 1 -49.

62. Koornneef M, Hanhart CJ and Thiel F. (1989). A genetic and phenotypic description of eceriferum (CER) mutants in Arabidopsis thaliana. The Journal of Heredity 80: $118-122$.

63. Krauss $P$, Markstädter C and Riederer M. (1997). Attenuation of UV radiation by plant cuticles from woody species. Plant, Cell and Environment 20; 1079-1085.

64. Krolikowski KA, Victor JL, Wagler TN, Lolle SJ and Pruitt RE. (2003). Isolation and characterization of the Arabidopsis organ fusion gene HOTHEAD. The Plant Journal 35: $501-511$.

65. Kunst $L$ and Samuels AL (2003) Biosynthesis and secretion of plant cuticular wax. Prog Lipid Res 42: 51-80.

66. Kurata T, Kawabata-Awai C, Sakuradani E, Shimizu S, Okada K and Wada T. (2003). The YORE-YORE gene regulates multiple aspects of epidermal cell differentiation in Arabidopsis. Plant J. 36: 55-66.

67. Kurdyukov S, Faust A, Nawrath C, Bär S, Voisin D, Efremova N, Franke R, Schreiber L, Saedler H, Métraux JP and Yephremov A. (2006a). The epidermisspecific extracellular BODYGUARD controls cuticle development and 
morphogenesis in Arabidopsis. Plant Cell 18: 321-339.

68. Kurdyukov S, Faust A, Trenkamp S, Bar S, Franke R, Efremova N, Tietjen K, Schreiber L, Saedler H and Yephremov A. (2006b). Genetic and biochemical evidence for involvement of HOTHEAD in the biosynthesis of long-chain alpha-, omega-dicarboxylic fatty acids and formation of extracellular matrix. Planta 224: 31529.

69. Lai C, Kunst L and Jetter R. (2007). Composition of alkyl esters in the cuticular wax on inflorescence stems of Arabidopsis thaliana cer mutants. The Plant Journal 50: 189-196.

70. Lechelt-Kunze C, Meissner RC, Drewes M and Tietjen K. (2003). Flufenacet herbicide treatment phenocopies the fiddlekead mutants in Arabidopsis thaliana. Pest Manag Sci. 59: 847-56.

71. Lee SB, Go YS, Bae HJ, Park JH, Cho SH, Cho HJ, Lee DS, Park OK, Hwang I and Suh MC. (2009a). Disruption of glycosylphosphatidylinositol-anchored lipid transfer protein gene altered cuticular lipid composition, increased plastoglobules, and enhanced susceptibility to infection by the fungal pathogen Alternaria brassicicola. Plant Physiology 150: 42-54.

72. Lee SB, Jung SJ, Kim HU, Kim JK, Cho HJ, Park OK and Suh MC. (2009b). Two Arabidopsis 3-ketoacyl CoA synthase genes, KCS20 and KCS2/DAISY, are functionally redundant in cuticular wax and root suberin biosynthesis, but differentially controlled by osmotic stress. Plant J. doi: 10.1111/j.1365$313 \times .2009 .03973 . x$

73. Li Y, Beisson F, Koo AJ, Molina I, Pollard M and Ohirogge J. (2007). Identification of 
acyltransferases required for cutin biosynthesis and production of cutin with suberinlike monomers. Proc. Nat. Acad. Sci. USA 104: 18339-18344.

74. Li F, Wu F, Lam P, Bird D, Zheng H, Samuels L, Jetter R and Kunst L. (2008). Identification of the Wax Ester Synthase/Acyl-CoA:Diacylglycerol Acyltransferase WSD1 required for Stem Wax Ester Biosynthesis in Arabidopsis thaliana. Plant cell 148: $97-107$.

75. Lippold F, Sanchez DH, Musialak M, Schlereth A, Scheible WR, Hincha DK and Udvardi MK. (2009). AtMyb41 regulates transcriptional and metabolic responses to osmotic stress in Arabidopsis. Plant Physiology 149: 1761-1772.

76. Lolle SJ, Berlyn GP, Engstrom EM, Krolikowski KA, Reiter WD and Pruitt RE. (1997). Developmental regulation of cell interactions in the Arabidopsis fiddlehead-1 mutant: a role for the epidermal cell wall and cuticle. Dev Biol. 189: 311-21.

77. Lu SY, Song T, Kosma DK, Parsons EP, Rowland O and Jenks M. (2009). Arabidopsis CER8 encodes long-chain acyl-CoA synthetase 1 (LACS1) that has overlapping functions with LACS2 in plant wax and cutin synthesis. Plant J. 59: 553564.

78. Lukowitz W, Gillmor CS and Scheible WR. (2000). Positional cloning in Arabidopsis. Why it feels good to have a genome initiative working for you. Plant Physiol. 123: 795-805.

79. Luque P, Bruque S and Heredia A. (1995). Water permeability of isolated cuticular membranes: a structural analysis. Archives of Biochemistry and Biophysics 317: 417-422. 
80. Macey MJK and Barber HN. (1970). Chemical genetics of wax formation on leaves of Pisum sativum. Phytochemistry 9: 5-12.

81. Macey MJK. (1974). Wax synthesis in Brassica oleracea as modified by trichloroacetic acid and glossy mutations. Phytochemistry 13: 1353-1358.

82. McNevin JP,Woodward W, Hannoufa A, Feldmann KA and Lemieux B. (1993). Isolation and characterization of eceriferum (cer) mutants induced by T-DNA insertions in Arabidopsis thaliana. Genome 36: 610-618.

83. Metz JG, Pollard MR, Anderson L, Hayes TR and Lassner MW. (2000). Purification of a jojoba embryo fatty acyl-Coenzyme A reductase and expression of its CDNA in high erucic acid rapeseed. Plant Physiology 122: 635-644.

84. Millar AA and Kunst L. (1997). Very-long-chain fatty acid biosynthesis is controlled through the expression and specificity of the condensing enzyme. The Plant Journal 12: $121-31$.

85. Millar AA, Clemens S, Zachgo S, Giblin EM, Taylor DC and Kunst L. (1999). CUT1, an Arabidopsis gene required for cuticular wax biosynthesis and pollen fertility, encodes a very-long-chain fatty acid condensing enzyme. Plant Cell 11: 825-838.

86. Mladenova K and Stoianova-Ivanova B. (1977). Composition of neutral com-ponents in flower wax of some decorative roses. Phytochemistry 16: 269-272.

87. Molina I, Ohlrogge JB and Pollard M. (2008). Deposition and localization of lipid polyester in developing seeds of Brassica napus and Arabidopsis thaliana. The Plant Journal 53: 437-449.

88. Nawrath C. (2006). Unraveling the complex network of cuticular structure and function. Curr Opin Plant Biol. 9: 281-7. 
89. Negruk V, Yang P, Subramanian M, McNevin JP and Lemieux B. (1996). Molecular cloning and characterization of the CER2 gene of Arabidopsis thaliana. Plant J. 9: $137-145$.

90. Ohlrogge JB, Jaworski JG and Post-Beittenmiller D. (1993). De novo fatty acid biosynthesis, in Lipid metabolism in plants (ed T.S. Moore Jr.), CRC Press, Boca Raton, pp. 3-32.

91. Ohlrogge $\mathrm{J}$ and Browse J. (1995). Lipid biosynthesis. Plant Cell 7:957-970.

92. Panikashvili D, Savaldi-Goldstein S, Mandel T, Yifhar T, Franke RB, Hofer R, Schreiver L, Chory J and Aharoni A. (2007). The Arabidopsis DESPERADO/AtWBC11 transporter in required for cutin and wax secretion. Plant Physiol. 145: 1345-60.

93. Peters JL, Cnudde F and Gerats T. (2003). Forward genetics and map-based cloning approaches. Trends Plant Sci. 8: 484-91.

94. Pighin JA, Zheng $H$, Balakshin LJ, Goodman IP,Western TL, Jetter R, Kunst $L$ and Samuels AL. (2004). Plant cuticular lipid export requires an ABC transporter. Science 306: 702-704.

95. Pinot F, Salaun JP, Bosch H, Lesot A, Mioskowski C and Durst F. (1992). omegaHydroxylation of Z9-octadecenoic, Z9,10-epoxystearic and 9,10-dihydroxystearic acids by microsomal cytochrome P450 systems from Vicia sativa. Biochemical and Biophysical Research Communications 184: 183-193.

96. Post-Beittenmiller D. (1996). Biochemistry and molecular biology of wax production in plants. Annual Review of Plant Physiology and Plant Molecular Biology 47: 405430. 
97. Pyee J, Yu H and Kolattukudy PE. (1994). Identification of a lipid transfer protein as the major protein in the surface wax of broccoli (Brassica oleracea) leaves. Archives of Biochemistry and Biophysics 311: 460-468.

98. Raffaele S, Vailleau F, Léger A, Joubès J, Miersch O, Huard C, Blée E, Mongrand S, Domergue F and Roby D. (2008). A MYB transcription factor regulates very-longchain fatty acid biosynthesis for activation of the hypersensitive cell death response in Arabidopsis. Plant Cell 20: 752-767.

99. Rashotte AM, Jenks MA and Feldmann KA. (2001). Cuticular waxes on eceriferum mutants of Arabidopsis thaliana. Phytochemistry 57: 115-123.

100. Rashotte AM, Jenks MA, Ross A and Feldmann KA. (2004). Novel eceriferum mutants in Arabidopsis thaliana. Planta 219: 5-13.

101. Ray AK, Chen Z, and Stark RE. (1998a). Chemical depolymerization studies of the molecular architecture of lime fruit cuticle. Phytochemistry 49: 65-70.

102. Ray AK and Stark RE. (1998b). Isolation and molecular structure of an oligomer produced enzymatically from the cuticle of lime fruit. Phytochemistry 48: 1313-1320.

103. Riederer M and Schreiber L. (2001). Protecting against water loss: analysis of the barrier properties of plant cuticles. J Exp Bot. 52: 2023-32.

104. Rogers DP and Bankaitis VA. (2000). Phospholipid transfer proteins and physiological functions. International Review of Cytology 197: 35-81.

105. Rossak M, Smith $M$ and Kunst L. (2001). Expression of the FAE1 gene and FAE1 promoter activity in developing seeds of Arabidopsis thaliana. Plant Mol Biol. 46: $717-25$.

106. Rowland O, Zheng H, Hepworth SR, Lam P, Jetter R and Kunst L. (2006). CER4 
encodes an alcohol-forming fatty acyl-Coenzyme A reductase involved in cuticular wax production in Arabidopsis. Plant Physiology 142: 866-77.

107. Rowland O, Lee R, Franke R, Schreiber L and Kunst L. (2007). The CER3 wax biosynthetic gene from Arabidopsis thaliana is allelic to WAX2/YRE/FLP1. FEBS Letters 581: 3538-3544.

108. Rupasinghe SG, Duan H and Schuler MA. (2007). Molecular definitions of fatty acid hydroxylases in Arabidopsis thaliana. Proteins 68: 279-93.

109. Samuels L, Kunst $L$ and Jetter R. (2008). Sealing plant surfaces: cuticular wax formation by epidermal cells. Annual Review of Plant Biology 59: 683-707.

110. Samuels L, DeBono A, Lam P, Wen M, Jetter R and Kunst L. (2008). Use of Arabidopsis eceriferum mutants to explore plant cuticle biosynthesis. J Vis Exp.

111. Schneider-Belhaddad F and Kolattukudy P. (2000). Solubilization, partial purification, and characterization of a fatty aldehyde decarbonylase from a higher plant, Pisum sativum. Archives of Biochemistry and Biophysics 377: 341-349.

112. Schnurr JA, Shockey JM, Boer GJ and Browse JA. (2002). Fatty acid export from the chloroplast. Molecualr characterization of a major plastidial acyl-Coenzyme A synthetase from Arabidopsis. Plant Physiology 129: 1700-1709.

113. Schnurr J, Shockey J and Browse J (2004) The acyl-CoA synthetase encoded by LACS2 is essential for normal cuticle development in Arabidopsis. The Plant Cell 16: $629-642$.

114. Schulz B and Frommer WB. (2004). A plant ABC transporter takes the lotus seat. Science 306: 622-625.

115. Shimakata T and Stumpf P. (1982). Isolation and function of spinach leaf $\beta$ - 
ketoacyl-[acylcarrier-protein] synthases. Proc. Nat. Acad. Sci. USA 79: 5808-5812.

116. Shockey JM, Fulda MS and Browse JA. (2002). Arabidopsis contains nine longchain acyl-coenzyme A synthetase genes that participate in Fatty acid and glycerolipid metabolism. Plant Physiology 129: 1710-1722.

117. Sieber P, Petrascheck M, Barberis A and Schneitz K. (2004). Organ polarity in Arabidopsis. NOZZLE physically interacts with members of the YABBY family. Plant Physiol. 135: 2172-85.

118. Somerville CR and Ogren WL. (1982). Mutants of the cruciferous plant Arabidopsis thaliana laking glycine decarboxylase activity. Biochem J. 202: 373-80.

119. Stark RE and Tian S. (2006). The cutin biopolymer matrix in Biology of The Plant Cuticle (eds M. Riederer and C. Müller), Blackwell Publishing, Oxford, pp. 126-144. 120. Stumpf P. (1984). Fatty acid biosynthesis in higher plants, in Fatty acid Metabolism and Its Regulation (ed. S Numa), Elsevier Sci, Amsterdam, pp. 155-79.

121. Suh MC, Samuels AL, Jetter R and Kunst L. (2005). Cuticular lipid composition, surface structure, and gene expression in Arabidopsis stem epidermis. Plant Physiology 139: 1649-1665.

122. Tanaka T, Tanaka H, Machida C, Watanabe M and Machida Y. (2003). A new method for rapid visualization of defects in leaf cuticle reveals five intrinsic patterns of surface defects in Arabidopsis. The Plant Journal 37: 139-146.

123. Tang D, Simonich MT and Innes RW. (2007). Mutations in LACS2, a long-chain acyl-coenzyme A synthetase, enhance susceptibility to avirulent Pseudomonas syringae but confer resistance to Botrytis cinerea in Arabidopsis. Plant Physiology 144: 1093-1103. 
124. Tegelaar EW, Kerp H, Visscher H, Schenck PA and Lee JW. (1991). Bias of the Paleobotanical Record as a Consequence of Variations in the Chemical Composition of Higher Vascular Plant Cuticles. Paleobiology 17: 133-144.

125. Thoma S, Kaneko $Y$ and Somerville C. (1993). A nonspecific lipid transfer protein from Arabidopsis is a cell wall protein. The Plant Journal 3: 427-436.

126. Todd J, Post-Beittenmiller D and Jaworski JG. (1999). KCS1 encodes a fatty acid elongase 3-ketoacyl-CoA synthase affecting wax biosynthesis in Arabidopsis thaliana. The Plant Journal 17: 119-130.

127. Ukitsu H, Kuromori T, Toyooka K, Goto Y, Matsuoka K, Sakuradani E, Shimizu S, Kamiya A, Imura Y, Yuguchi M, Wada T, Hirayama T and Shinozaki K. (2008). Cytological and biochemical analysis of COF1, an Arabidopsis mutant of an $A B C$ transporter gene. Plant and Cell Physiology 48: 1524-1533.

128. Villena FJ, Domınguez E and Heredia A. (2000).Monitoring biopolymers present in plant cuticles by FT-IR spectroscopy. Journal of Plant Physiology 156: 419-422.

129. Vioque $\mathrm{J}$ and Kolattukudy PE. (1997). Resolution and purification of an aldehydegenerating and an alcohol-generating fatty acyl-CoA reductase from pea leaves (Pisum sativum L.). Archives of Biochemistry and Biophysics 340: 64-72.

130. Voelker TA. (1996). Plant acyl-ACP thioesterases: chain-length determining enzymes in plant fatty acid biosynthesis. Genetic Engineering 18: 111-131.

131. von Wettstein-Knowles P. (1979). Genetics and biosynthesis of plant epicuticular waxes. In Advances in the biochemistry and physiology of plant lipids pp. 1-26.

132. von Wettstein-Knowles P. (1982). Elongase and epicuticular wax biosynthesis. Physiologie Végétale 20:797-809. 
133. von Wettstein-Knowles P. (1993). Waxes, cutin and suberin, in Lipid Metabolism in Plants (ed. T Moore), FL: CRC Press, Boca Raton, pp. 127-66.

134. Wellesen K, Durst F, Pinot F, Benveniste I, Nettesheim K, Wisman E, SteinerLange S, Saedler H and Yephremov A. (2001). Functional analysis of the LACERATA gene of Arabidopsis provides evidence for different roles of fatty acid hydroxylation in development. Proc. Nat. Acad. Sci. USA 98: 9694-9699.

135. Xia Y, Nikolau BJ and Schnable PS. (1997). Developmental and hormonal regulation of the Arabidopsis CER2 gene that codes for a nuclear-localized protein required for the normal accumulation of cuticular waxes. Plant Physiology 115: 925937.

136. Xiao F, Goodwin SM, Xiao Y, Sun Z, Baker D, Tang X, Jenks MA and Zhou JM. (2004). Arabidopsis CYP86A2 represses Pseudomonas syringae type III genes and is required for cuticle development. The EMBO Journal 23: 2903-2913.

137. Xu X, Dietrich CR, Lessire R, Nikolau BJ and Schnable PS. (1997). The endoplasmic reticulum-associated maize GL8 protein is a component of the acyl CoenzymeA elongase involved in the production of cuticular waxes. Plant Physiolology 128: 924-934.

138. Xu X, Dietrich CR, Lessire R, Nikolau BJ and Schnable PS. (2002). The endoplasmic reticulum-associated maize gl8 protein is a component of the acyl-CoA elongase involved in the production of cuticular waxes. Plant Physiology 128: 924934.

139. Zheng $H$, Rowland $O$ and Kunst L. (2005). Disruptions of the Arabidopsis enoylCoA reductase gene reveal an essential role for very-long-chain fatty acid synthesis 
in cell expansion during plant morphogenesis. Plant Cell 17: 1467-1481.

140. Zlotnik-Mazori T and Stark RE. (1988). Nuclear magnetic resonance studies of cutin, an insoluble plant polyester. Macromolecules 21: 2412-2417. 